San Jose State University

SJSU ScholarWorks

Master's Theses

Master's Theses and Graduate Research

Summer 2013

\title{
Effect of nanodroplet ink concentration on image contrast for reverse-emulsion electrophoretic displays
}

Winston Kuantung Wang

San Jose State University

Follow this and additional works at: https://scholarworks.sjsu.edu/etd_theses

\section{Recommended Citation}

Wang, Winston Kuantung, "Effect of nanodroplet ink concentration on image contrast for reverseemulsion electrophoretic displays" (2013). Master's Theses. 4369.

DOI: https://doi.org/10.31979/etd.h6m6-brcm

https://scholarworks.sjsu.edu/etd_theses/4369

This Thesis is brought to you for free and open access by the Master's Theses and Graduate Research at SJSU ScholarWorks. It has been accepted for inclusion in Master's Theses by an authorized administrator of SJSU ScholarWorks. For more information, please contact scholarworks@sjsu.edu. 


\title{
EFFECT OF NANODROPLET INK CONCENTRATION ON IMAGE CONTRAST FOR REVERSE-EMULSION ELECTROPHORETIC DISPLAYS
}

\author{
A Thesis \\ Presented to \\ The Faculty of Department of Mechanical \& Aerospace Engineering \\ San José State University
}

\author{
In Partial Fulfillment \\ of the Requirements for the Degree \\ Master of Science
}

by

Winston Kuantung Wang

August 2013 
(c) 2013

Winston Kuantung Wang ALL RIGHTS RESERVED 
The Designated Thesis Committee Approves the Thesis Titled

EFFECT OF NANODROPLET INK CONCENTRATION ON IMAGE CONTRAST FOR REVERSE-EMULSION ELECTROPHORETIC DISPLAYS

by

Winston Kuantung Wang

APPROVED FOR THE DEPARTMENT OF MECHANICAL \& AEROSPACE ENGINEERING

\section{SAN JOSÉ STATE UNIVERSITY}

August 2013

Dr. Sang-Joon John Lee

Department of Mechanical and Aerospace Engineering

Dr. Maryam Mobed-Miremadi

Department of General Engineering

Dr. Mateusz Bryning

Zikon Inc. 


\section{ABSTRACT \\ EFFECT OF NANODROPLET INK CONCENTRATION ON IMAGE CONTRAST FOR REVERSE-EMULSION ELECTROPHORETIC DISPLAYS by Winston Kuantung Wang}

Reverse-emulsion electrophoretic display technology is based on an electro-responsive ink comprised of self-assembled nanodroplets dispersed in a non-polar liquid. The dye-containing nanodroplets are selectively driven toward or away from the viewing plane of a display by electric fields. The hypothesis of this study is that image contrast in a nanodroplet electrophoretic display is governed by concentration and steric effects that limit the intensity of the dark state.

Simultaneously, steric effects as well as electrostatic screening can diminish whiteness in the light state. This hypothesis has been tested by multiphysics simulation of dilute species in electrostatic fields and experimental measurements of relative luminance in test displays. Concentration level was varied in a range of dilutions from full concentration (100\%) to one-eighth (12.5\%) and the highest contrast ratio was achieved at $25 \%$. The test devices exhibited behavior that was similar to the saturation effects predicted by simulation, accounting for steric effects. Ink concentration showed little effect on switching time, reaching steady-state within approximately 2 seconds for all concentration levels. The hypothesis was further tested by experimentally observing the effect of driving voltage between $1 \mathrm{~V}$ to $8 \mathrm{~V}$. The results showed no significant improvement of contrast even at higher voltage, further suggesting that concentration and steric effects dictate maximum contrast. 


\section{ACKNOWLEDGEMENTS}

This work was supported by Award IIP-1143479 from the National Science Foundation. It has been a great privilege to spend my last year at San Jose State University (SJSU) in the Microelectromechanical Systems Laboratory. First and foremost, I owe my deepest gratitude to my research advisor and committee chair, Dr. Lee, who has supported and guided me throughout this thesis. He not only gave me the opportunity to conduct research within his group but also taught me the meaning of professionalism. This thesis would not be possible without his encouragement and direction. I would also like to thank Dr. Bryning for his friendly nature, immense knowledge, and valuable discussions. He was the Ironman that provided me with the tools to make data acquisition possible. Our constant laughter was what kept my dreams and spirits high. Next, I would like to thank Dr. Maryam Mobed-Miremadi. She is one of the kindest professors. She constantly provided new ideas, guidance, and encouragement to pursue a higher education degree. To my loving friends, it has been a great journey. I enjoyed our laughter, joy, and overnight projects with less than healthy food. A very special thanks to Will Schulkins for his invaluable assistance with dynamic light scattering measurements. I offer my sincere appreciation to Remy Cromer for giving me the opportunity to work with his nanodroplet ink. Finally, I would like to dedicate this work to my parents, Danny and Viola. This thesis is tangible evidence of my appreciation for my friends and family. Thank you for never doubting my ability, for your constant support, and for your unconditional love. It has provided me strength and willpower to complete this challenge and has helped me become a better person. 


\section{TABLE OF CONTENTS}

\section{CHAPTER}

1 INTRODUCTION 1

1.1 Electronic paper . . . . . . . . . . . . . . . . . . . . 1

1.2 Reverse-emulsion electrophoretic displays . . . . . . . . . . . . . . 2

1.2.1 Commercial merit . . . . . . . . . . . . . . . . . . . . 4

1.3 Hypothesis . . . . . . . . . . . . . . . . . 6

1.4 Significance .......................... 8

1.5 Research approach . . . . . . . . . . . . . . . . . . . 9

2 RELATED WORK 10

2.1 Related research on microencapsulated electrophoretic display technology 10

2.2 Alternative electronic display technologies . . . . . . . . . . . 13

2.2.1 Electrowetting display technology . . . . . . . . . . . . 13

2.2.2 Liquid powder display technology . . . . . . . . . . . . . . 15

2.2.3 Electrochromic display technology . . . . . . . . . . . . . . . . 15

2.2.4 Microcup ${ }^{\circledR}$ electrophoretic technology . . . . . . . . . . . . . 17

2.2.5 Electrokinetic display technology . . . . . . . . . . . . . 18

2.3 Related research work on nanodroplet electrophoretic devices . . . . . 19

3 THEORY 22

3.1 Force order of magnitude comparison . . . . . . . . . . . . . . . . . 22

3.2 Electrostatic force and electrophoresis phenomena . . . . . . . . . 23

3.3 Electric double layers . . . . . . . . . . . . . . . . . . 25 
3.4 Poisson-Nernst-Planck modeling . . . . . . . . . . . . . . . . 28

4 INK CHARACTERIZATION 31

4.1 Nanodroplet ink concentration . . . . . . . . . . . . . . . . . 31

4.2 Nanodroplet charge estimation . . . . . . . . . . . . . . . . . . 31

4.3 Nanodroplet size measurement and morphology . . . . . . . . . . . . 31

4.4 Atomic force microscopy . . . . . . . . . . . . . . . . . . 33

4.5 Dynamic light scattering . . . . . . . . . . . . . 34

4.6 Ink material properties . . . . . . . . . . . . . . 35

4.6.1 Ink permittivity measurement . . . . . . . . . . . 36

4.6.2 Ink conductivity measurement . . . . . . . . . . . . . . 37

4.7 Porous medium structure . . . . . . . . . . . . . . . . 37

5 NUMERICAL SIMULATION 40

5.1 Model setup and information . . . . . . . . . . . . . 41

5.2 Effect of nanodroplet ink concentration on display contrast . . . . . . 41

5.2.1 Nanodroplet concentration at the viewing plane . . . . . . . . 43

5.2.2 Electric potential vs. distance from electrode . . . . . . . . . 44

5.2.3 Steric compensation ................. . . 44

5.3 1-D vs. 2-D: Role of geometry in simulation . . . . . . . . . . . 48

5.3.1 3-D computed tomography . . . . . . . . . . . . . . 49

5.4 Transient analyses of display switching . . . . . . . . . . . 50

5.4.1 Effects of voltage on display switching . . . . . . . . . . 50

5.4.2 Effect of nanodroplet ink concentration on display switching . 51

5.5 Parametric simulation of display switching . . . . . . . . . . 54 
6 EXPERIMENTAL TESTING 57

6.1 Luminosity measurement . . . . . . . . . . . . . . . 57

6.2 Ink concentration experiment . . . . . . . . . . . 58

6.3 Voltage experiment . . . . . . . . . . . . . . . 60

7 RESULTS AND DISCUSSION 61

7.1 Image contrast . . . . . . . . . . . . . . . . . 61

7.2 Switching speed . . . . . . . . . . . . . . 61

7.3 Spatial variability . . . . . . . . . . . . . 63

7.4 Comparison with simulation . . . . . . . . . . . 64

7.5 Voltage experiments ... . . . . . . . . . . . 64

8 CONCLUSIONS AND FUTURE WORK 68

$\begin{array}{ll}\text { REFERENCES } & 70\end{array}$

APPENDIX

A STEADY STATE DATA FROM CONCENTRATION EXPERIMENT 84

B TRANSIENT DATA FROM CONCENTRATION EXPERIMENT 92

$\begin{array}{lll}\text { C } & \text { DATA FROM VOLTAGE EXPERIMENT } & 97\end{array}$

D DATA ANALYSIS OF THE TRANSIENT VOLTAGE EXPERIMENT 106 


\section{LIST OF FIGURES}

Figure

1 Reverse-emulsion droplet. . . . . . . . . . . . . . . . 3

2 The operating principle of a single-pixel reverse-emulsion electrophoretic display. . . . . . . . . . . . . . . . . . 5

3 Illustration of the 2 extreme concentration scenarios. . . . . . . . . 7

4 Hypothesized concentration effect on display contrast outcome. . . . . 8

5 The operating principle of a microencapsulated electrophoretic display. 12

$6 \quad$ Power consumption comparison among different display technologies. 13

7 The operating principle of an electrowetting display. . . . . . . . . . . 14

8 The operating principle of a liquid powder display . . . . . . . . . . 16

9 The operating principle of an electrochromic device. . . . . . . . . . . 17

10 Microcup ${ }^{\circledR}$ profile of SiPix electrophoretic display. . . . . . . . . . . . 18

11 Microcup ${ }^{\circledR}$ display and driving voltage. . . . . . . . . . . . . . . . 18

12 Examples of electrokinetic display technology. . . . . . . . . . . . . . 19

13 Visualization of the forces acting on a ink nanodroplet. . . . . . . . 25

14 Visualization of an electric double layers. . . . . . . . . . . . . 26

15 Atomic force microscopy of dried nanodroplets. . . . . . . . . . . . . 34

16 Oblique view of dried nanodroplets via atomic force microscopy. . . . 35

17 Nanodroplet size distribution by dynamic light scattering . . . . . . . 35

18 Current-voltage plot for a reverse-emulsion electrophoretic display. . . 38 
19 Scanning electron microscopy image of dry powder, and bonded substrates. . . . . . . . . . . . . . . . . . . . 38

20 Concentration effect on display contrast 1-D simulation informations

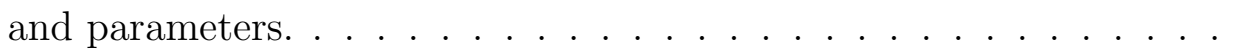

21 Concentration as a function of distance from electrode, for nanodroplets and counterions, at different levels of bulk concentration. . . . . . . . 44

22 Electric potential versus distance from electrode, for a range of bulk concentration. . . . . . . . . . . . . . . . . . 45

23 Poisson-Nernst-Planck model of nanodroplet concentration near the viewing plane in dark state and light state . . . . . . . . . . 46

24 A unit cell as the base of the 2-D hexagonal porous lattice. . . . . . 48

25 Electrostatic screening comparison at the electrode of 1-D and 2-D hexagonal porous models. . . . . . . . . . . . . . . . . . . . . 49

26 List of information and parameters for the 1-D and 2-D voltage effects on display switching models. . . . . . . . . . . . . . . . . . . . 51

27 Ink concentration as a function of time and potential voltage at the electrode for 1-D (left) and 2-D (right) simulations. . . . . . . . 51

28 List of information and parameters for the 1-D and 2-D concentration effects on display switching models. . . . . . . . . . . . . . . 52

29 Ink nanodroplet concentration as a function of time and bulk concentration at the electrode for a 1-D and 2-D simulation. . . . . . . . 53

30 Ink nanodroplet concentrations at the electrode reaching steady-state for different bulk concentrations with the same voltage potential. . . . 54

31 Particle Trajectories Simulation. . . . . . . . . . . . . 56 
32 Distance normalized device response comparison for different combi-

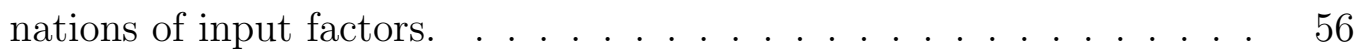

33 Circuit connections for the data acquisition device. . . . . . . . 58

34 Luminance testing aperture with light source and photodiode. . . . . 59

35 Relative luminance versus ink concentration. . . . . . . . . . . . 62

36 Contrast versus ink concentration. . . . . . . . . . . . . . . . 62

37 Relative luminance versus time for two light-to-dark switching transitions, using different ink concentrations. . . . . . . . . . 63

38 Variability in relative luminance at three distinct locations per device, for each concentration level. . . . . . . . . . . . . 65

39 Comparison of the simulation prediction and experimental result. . . 66

40 Relative luminance versus applied voltage with devices at the same

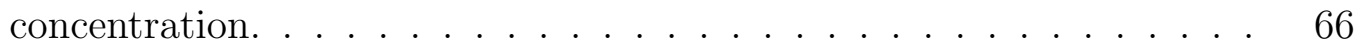




\section{LIST OF SYMBOLS}

k Boltzmanns constant . . . . . . . . . . . . . . 22

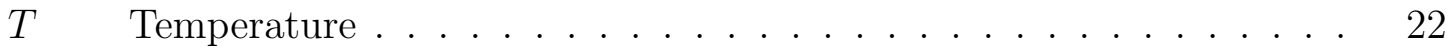

a Representative particle length . . . . . . . . . . . . . 22

$\mathrm{A}_{\text {eff }}$ Hamaker constant . . . . . . . . . . . . . . 22

$\mu \quad$ Dynamic viscosity . . . . . . . . . . . . . . 23

U Particle velocity . . . . . . . . . . . . . . 23

$\rho \quad$ Fluid density . . . . . . . . . . . . . . . . . 23

$q_{1} \quad$ Charge of particle $1 \ldots \ldots \ldots 23$

d Distance between charged particles . . . . . . . . . . . 23

$k_{e} \quad$ Distance between charged particles . . . . . . . . . . 23

$\varepsilon_{r} \quad$ Relative permittivity .................... 24

$\varepsilon_{0} \quad$ Permittivity of space ...................... 24

$\zeta \quad$ Zeta potential/ electrokinetic potential .......... 24

$\mu_{m} \quad$ Ion mobility . . . . . . . . . . . . . . . . . . . . . . . 24

$\Psi_{0} \quad$ Applied potential . . . . . . . . . . . . . . 26

$\Psi_{s} \quad$ Effective potential . . . . . . . . . . . . . 26

$\varphi \quad$ Electrostatic potential . . . . . . . . . . . . 28

$Z_{i} \quad$ Charge number of species $i \ldots \ldots \ldots . \ldots 28$

$J_{i} \quad$ Flux of ion specie $i \ldots \ldots \ldots$. . . . . . . . . . . . . 29

$D_{i} \quad$ Diffusivity of the ion specie $i \ldots \ldots$. . . . . . . . . . . . 29

R Universal gas constant . . . . . . . . . . . . . . . 29

$\mathrm{N}_{\mathrm{A}} \quad$ Avogadro's number ...................... 29 
r Nanodroplet radius . . . . . . . . . . . . . . . . . . 29

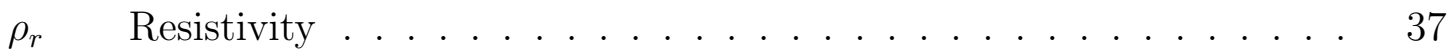

$R_{r} \quad$ Electrical resistance . . . . . . . . . . . . . 37

$A_{s} \quad$ Cross-sectional area . . . . . . . . . . . . . . . . . . 37

l Material length . . . . . . . . . . . . . . . 37 


\section{CHAPTER 1}

\section{INTRODUCTION}

\section{$1.1 \quad$ Electronic paper}

In recent years, electronic paper displays have played a significant role in the display market, because of their low power consumption and their paper-like appearance with ambient-light readability and wide viewing angles. The most common electronic paper technology on the market is the microencapsulated electrophoretic display. This technology is used in e-readers, e.g., Amazon Paperwhite and the Barnes \& Noble NOOK [1]. However, electronic paper displays based on microencapsulated electrophoretic particle technology are incapable of streaming videos because of the low refresh rates and the ghosting effect (shadows from previous image) $[2,3,4,5]$. Also, it is difficult to introduce vivid full color through microencapsulated electrophoretic capsules without increasing power consumption or reducing the resolution. Enabling colors in current microencapsulated electrophoretic technology must also solve the problem of color desaturation $[6,7,8]$. The reverse-emulsion electrophoretic display technology developed by Zikon, Inc. (Sunnyvale, California, USA) has inherent functional advantages that may enable it to overcome these limitations. A reverse-emulsion electrophoretic display is fundamentally similar to conventional paper and ink. The two main functional components of a reverse-emulsion electrophoretic display are ink and a white porous medium, which produce contrast similar to that of conventional paper. A reverse-emulsion electrophoretic display device costs less to manufacture compared to competing technologies (e.g., liquid crystal display [LCD] and other 
electronic paper displays). Some commercial applications of the reverse-emulsion electrophoretic display technology include e-readers, traffic signage, digital medical records, retail and advertising labels, and transparent and flexible displays.

\subsection{Reverse-emulsion electrophoretic displays}

Reverse-emulsion electrophoretic display technology, developed by Zikon Inc., was first introduced in 1998 [9]. The technology revolves around a porous matrix and reverse-emulsion ink nanodroplets powered by electrophoresis. The ink consist of a polar fluid and dissolved dye stabilized by surfactants. The nanodroplets are suspended in a transparent non-polar fluid, thus creating a reverse-emulsion. Figure 1 shows an idealized rendering of nanodroplet morphology and the stabilizing surfactants. The reverse micelles are hydrophobic to the surrounding solution, with the hydrophobic tails pointing outward and the hydrophilic heads facing inward, surrounding the polar dye $[10,11]$. Individual micelles are responsive to an applied electric potential caused by charge generation from the nanodroplet dye and surfactants. The details of nanodroplet charge is investigated in section 4.2. In this study reverse micelles in a reverse-emulsion electrophoretic display are referred to as nanodroplets. The nanodroplets are thermodynamically stable, low-cost, and environmentally friendly, and they self-assemble with mix-and-stir manufacturing. Nanodroplets are an order of magnitude smaller than conventional microencapsulated electrophoretic capsules, so their response to an electric field is expected to be relatively faster. The white porous matrix can be manufactured at low-cost, using reel-to-reel and spray deposition methods on flexible substrates. Studies have shown that a reverse-emulsion electrophoretic display can provide a transmissivity in excess $70 \%$, a wide viewing angle, bright colors, and low power 
consumption $[12,13]$.

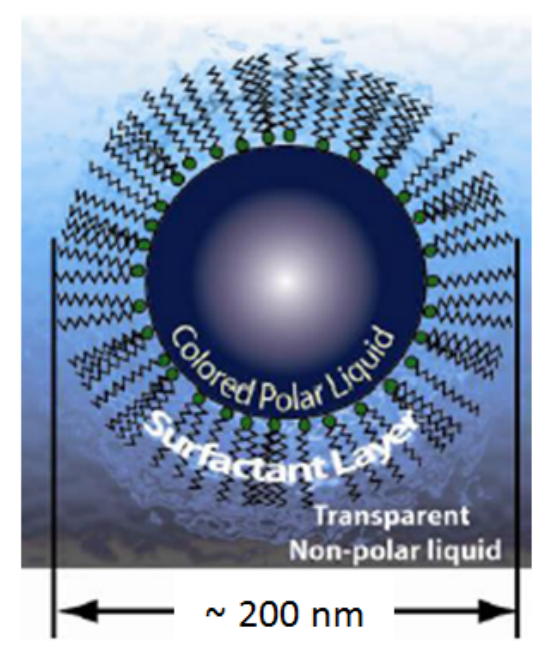

Figure 1: Illustration of reverse-emulsion droplet. Image courtesy of Zikon Inc. [14]

The functionality of a reverse-emulsion electrophoretic display ink device is tested by placing the ink nanodroplets within 2 glass slides coated with indium tin oxide (ITO) (Delta Technologies, Ltd., Loveland, Colorado, USA). As the electric fields are established between the 2 electrodes, ink nanodroplets respond to the electric fields similar to a charged particle driven by electrostatic force. The ink nanodroplets are an order of magnitude smaller than microencapsulated capsules that are used in current e-paper displays. Zikon Inc. has estimated the response time of a reverse-emulsion electrophoretic display to be less than $30 \mathrm{~ms}$ [15], based on the fact that the ink nanodroplets are an order of magnitude smaller than microencapsulated capsules. The faster response time gives the reverse-emulsion electrophoretic display significant advantages over microencapsulated electrophoretic displays. Furthermore, unlike the microencapsulated electrophoretic display and other emissive displays, a reverse-emulsion electrophoretic display requires only low-cost, environmental-friendly, nontoxic materials. 
Similar to a sheet of white paper, contrast is achieved by the difference in color between dark ink and a white porous matrix. The white porous matrix, in which the ink is loaded, is formed by packing micron-scale $\mathrm{TiO}_{2}$ particles. Between $\mathrm{TiO}_{2}$ particles, void spaces are formed. In the dark state, dye-containing nanodroplet ink can be seen at the viewing plane when the electric field drives the ink toward it through the porous matrix. In contrast, when the voltage current drives the ink away from the viewing surface, the white porous matrix allows the ink to become obfuscated within the porous matrix. Ideally, the device is at its best lightness when only the white porous matrix is visible. Recent studies have demonstrated bistability and good uniformity achieved at low voltage, depending on the composition of the porous matrix [13]. Figure 2 illustrates a cross-sectional view of a single-pixel display switching from a light to dark state.

\subsubsection{Commercial merit}

Low-cost electronic paper is favorable for applications such as electronic shelf labels (ELSs) [16]. Besides providing new and fresh goods, grocery stores must stay competitive by responding quickly to consumer needs, by becoming more innovative toward bringing in new products, and by being more efficient with accommodating store growth [17, 18]. In 2012, the Food Marketing Institute counted more than 37,000 supermarkets that provide a full line of groceries, meat and produce. These stores can carry anywhere from 15,000 to 60,000 stock keeping units (SKUs).

Typically, each product requires one ESL. This corresponds to a potential market of 1.6 billion ESLs in U.S. grocery supermarkets alone [19]. The digital pricing provides shops with the ability to change prices more quickly and efficiently [20]. If the paper labels were to be replaced by ESL, managers would be able to review and update thousands of products instantly at their desks. Store employees would be 

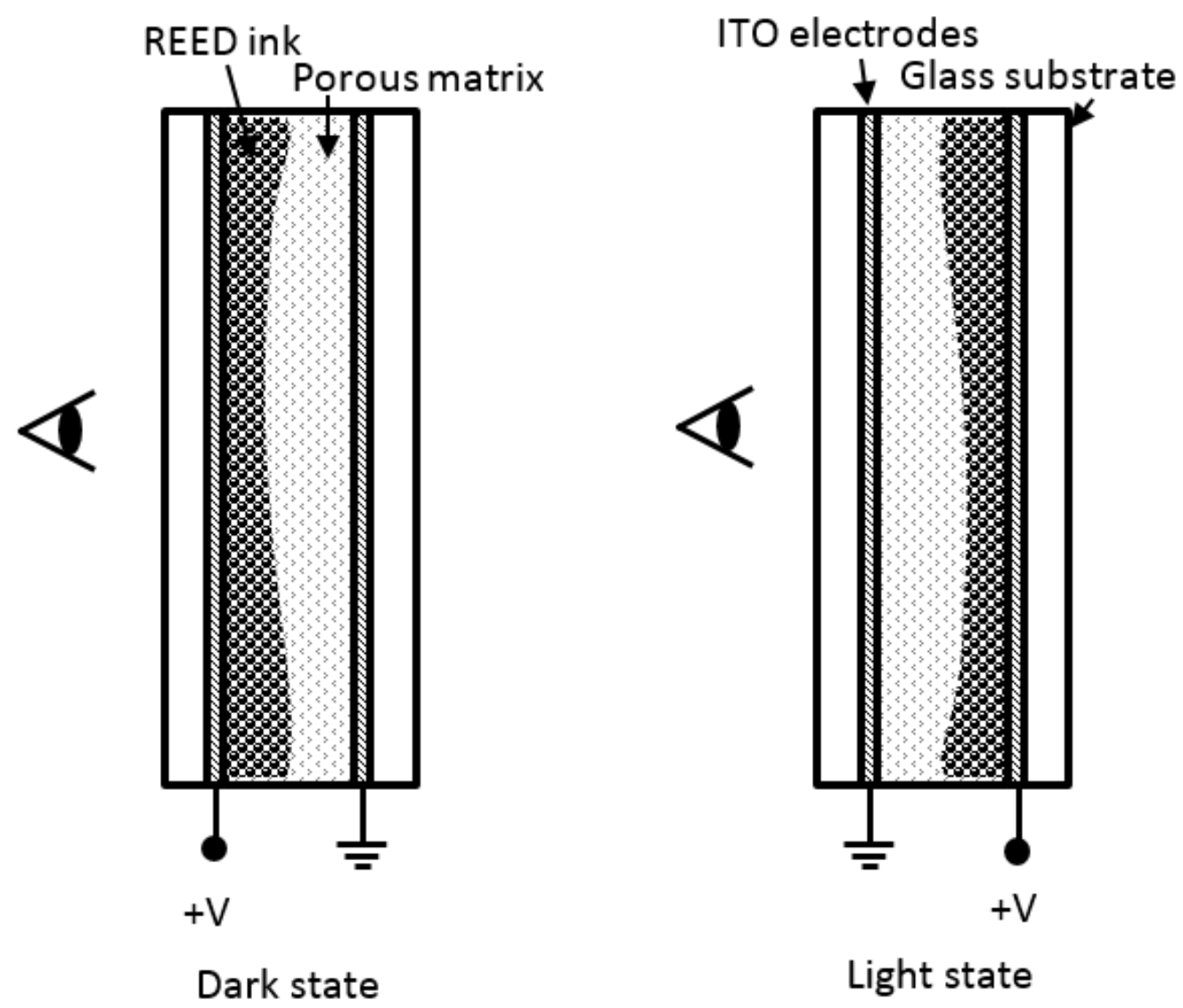

Figure 2: The operating principle of a single pixel reverse-emulsion electrophoretic display. Left of the image illustrates the device at dark state, with ink nanodroplets driven toward the viewing plane by voltage potential. While the right of the image illustrates the light state, with ink nanodroplets driven away from the viewing plane by reversing the voltage potential.

able to provide more personal help and directly interact with customers. Smart electronic shelf labels provide more information about products and inventory in real time. Flexible and strategic pricing provide the ability to start and end promotions at the exact time to maximize sales. 


\section{$1.3 \quad$ Hypothesis}

Contrast is an important aspect of every visual display system. An image with low contrast is difficult to see, and may cause eye strain. Studies have shown that a reverse-emulsion electrophoretic display, similar to most electronic paper displays, is capable of displaying excellent contrast [15]. To this researcher's knowledge, no research has been done to optimize the contrast of the display nor to understand the physics behind improving the contrast and switching response. This study addresses and identifies the scientific gap in knowledge regarding concentration effect on image contrast in a reverse-emulsion electrophoretic display. The hypothesis of this research is as follows: Concentration of the nanodroplet ink within a reverse-emulsion electrophoretic display is the primary factor that determines maximum contrast. Image contrast in a nanodroplet electrophoretic display is governed by steric effects that limit the performance of the dark state.

Simultaneously, both the steric effects and electrostatic screening limit the performance of the light state.

When a device has very low ink concentration, ink nanodroplets are able to withdraw into the porous matrix in the light state. The resulting image at the viewing plane is as white as the $\mathrm{TiO}_{2}$ layer. However, as figure 3(a) illustrates, the device has low contrast in the dark state because of insufficient ink nanodroplets (within the ink solution) to occupy the viewing plane. In the case of very high ink concentration, the concentration of the ink occupies all the void cavities, which makes it impossible for the nanodroplets to migrate and diffuse into the microchannels. The dark state is dark because of the high density of dye-carrying ink nanodroplets spreading everywhere within the device. Figure 3(b) illustrates the switching to a light state by driving the dye-carrying ink nanodroplets away from 
the viewing plane.

Thus, the hypothesis suggests that when a pixel is set to the dark state an optimal ink concentration can achieve effective saturation of the viewing plane. If the hypothesis is correct, this work will provide knowledge on nanodroplet ink concentration for optimum contrast in a reverse-emulsion electrophoretic display system.

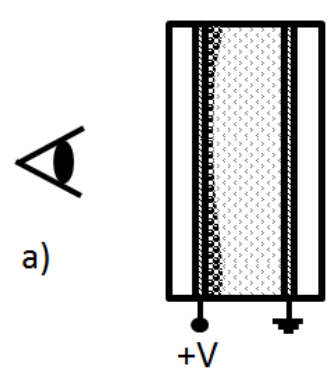

Dark

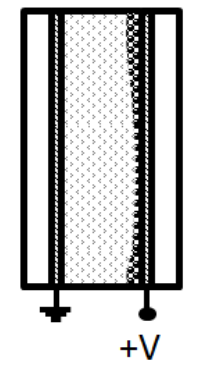

Light

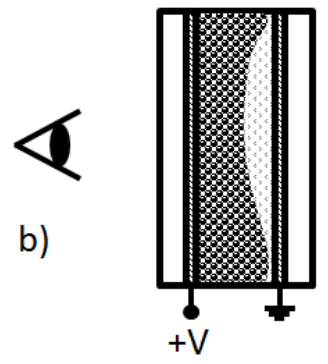

Dark

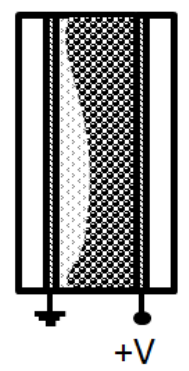

Light

Figure 3: Illustration of the 2 extreme concentration scenarios. (a) Extreme low concentration. (b) Extreme high concentration.

A anticipated outcome based on the steric effects and electrostatic screening is shown in figure 4 based on the hypothesis statement. The best contrast in the display can be found somewhere between the first and second extreme concentration scenarios. The maximum darkness is limited by ink nanodroplets that can be packed within the void matrix. Increasing concentration beyond the packing limit will not improve darkness. Excess ink nanodroplets within the device result in total electrostatic screening, which hinders maximum lightness. 


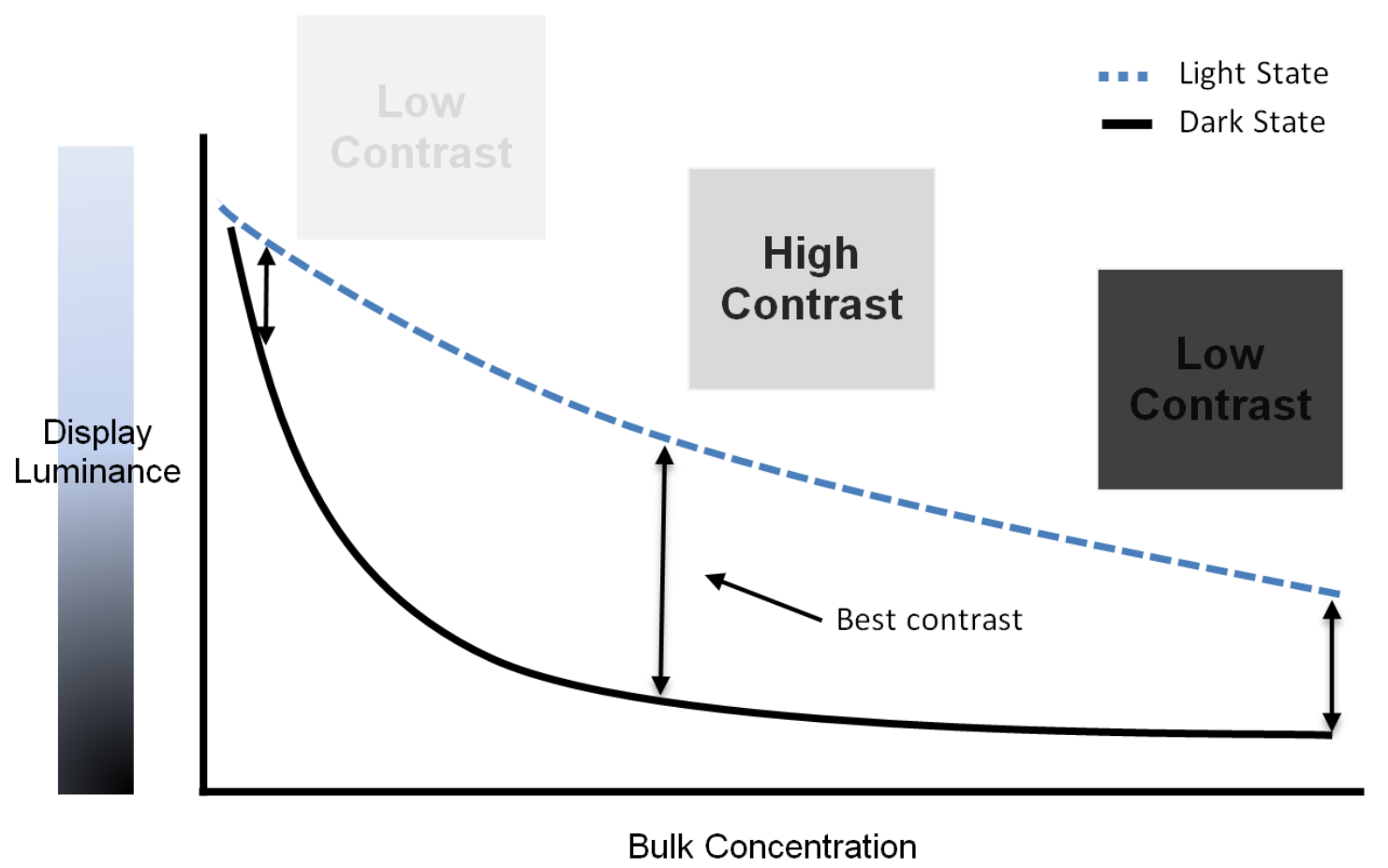

Figure 4: Predicted contrast behaviors based on the hypothesis at different ink concentrations are illustrated by the ratio of peak light and dark states.

\subsection{Significance}

The extent to which this hypothesis is true will improve the ability to design and develop electronic paper displays with high contrast. The benefit of developing this type of display will provide a viable alternative that has lower environmental impact than conventional displays, most of which require resource-intensive microfabrication processes. E-paper devices consume much less power than most conventional displays and, if more widely adopted, can reduce energy demand and consumption. Intuitively, emissive displays can't be good when exposed for long periods of time. However, in today's society, children are keen on them at a much younger age. This has resulted in a surge in nearsightedness and other related eye diseases [21]. 


\subsection{Research approach}

Characterization of the ink-carrying nanodroplets is the first step in supporting the predictive models with accurate information. A predictive model of charged nanodroplet response in a porous medium under an applied electric field is explored using multiphysics software simulation. Empirical data are collected from single-pixel test specimens, and the data are used to refine and verify the predictive model. Steady-state analysis is sufficient for determining the peak contrast of the display from values obtained in the peak light and dark states. Transient studies of the display switching response are included in both simulation and experimental data. The transient analysis of the display switching is included in the chapter on future work so that the switching response can be carefully studied in the future. 


\section{CHAPTER 2}

\section{RELATED WORK}

The related work chapter is divided into three sections. The technical merit of microencapsulated electrophoretic display technology is investigated in the first section. The second section discusses alternative electronic paper display technologies that are under research and development. The third section covers the research of electrophoretic displays similar to the reverse-emulsion electrophoretic display.

2.1 Related research on microencapsulated electrophoretic display technology

Microencapsulated electrophoretic display technology was first introduced in a patent by researchers from Massachusetts Institute of Technology in 1998 [22, 23]. The orientation of electrophoretic material can be determined by applying voltage across the microencapsulated capsules. When a voltage is applied on a pixel, the negatively charged white particles inside the microencapsulated capsules arrange themselves to become closer to the positively charged electrode. Positively charged black particles rearrange themselves toward the negatively charged electrode, similar to the negatively charged white particles. When viewed on the positively charged electrode, only the white particles attracted to the positively charged electrode are seen. Viewing from the same spot, a dark pixel can be seen by reversing the voltage. An image is formed based on how the voltage is applied. The operating principle of a microencapsulated device is shown in figure 5 .

Electronic paper displays mimic the best aspects of conventional ink and 
paper [24]. Unlike emissive displays, electronic paper displays deliver information using contrast difference by varying the reflectance of different frequencies of light. Since backlight is not needed in reflective displays, the energy consumption of electronic paper display is far less than that of emissive display technology (e.g., CRT and LED). An electronic paper display with microencapsulated electrophoretic technology can also achieve bistability without additional power support, which is another distinct advantage. The capsules within the microencapsulated devices do not rearrange their orientation easily after the image is formed. This, in turn, makes bistability ideal for e-readers and many other applications. Power is no longer needed to maintain the image once a page is loaded. Bistable reflective displays consume much less power than emissive displays; often, a device can function for months before recharging [25]. Figure 6 shows power consumption comparison among different display technologies. Reflective displays require power only when switching between pages or frames, whereas emissive displays consume energy continuously even for stationary images.

In contrast, electronic paper display images are created by lights reflecting off the active matrix. Image contrast and viewing angle improve as the ambient light increase [27]. Studies have been done on eye fatigue for different displays to demonstrate that electronic paper display is the best for reading [28, 29]. A study in Japan concluded that there is no significant difference in the level of visual fatigue between reading with an electronic paper display versus reading with conventional paper [30]. This is the main quality that electronic paper displays has to offer to consumers. Electronic paper display can serve as a rewritable and long-lasting alternative to conventional wood-based paper to reduce global consumption of natural resources.

Electronic paper displays also have disadvantages. Bistability of 


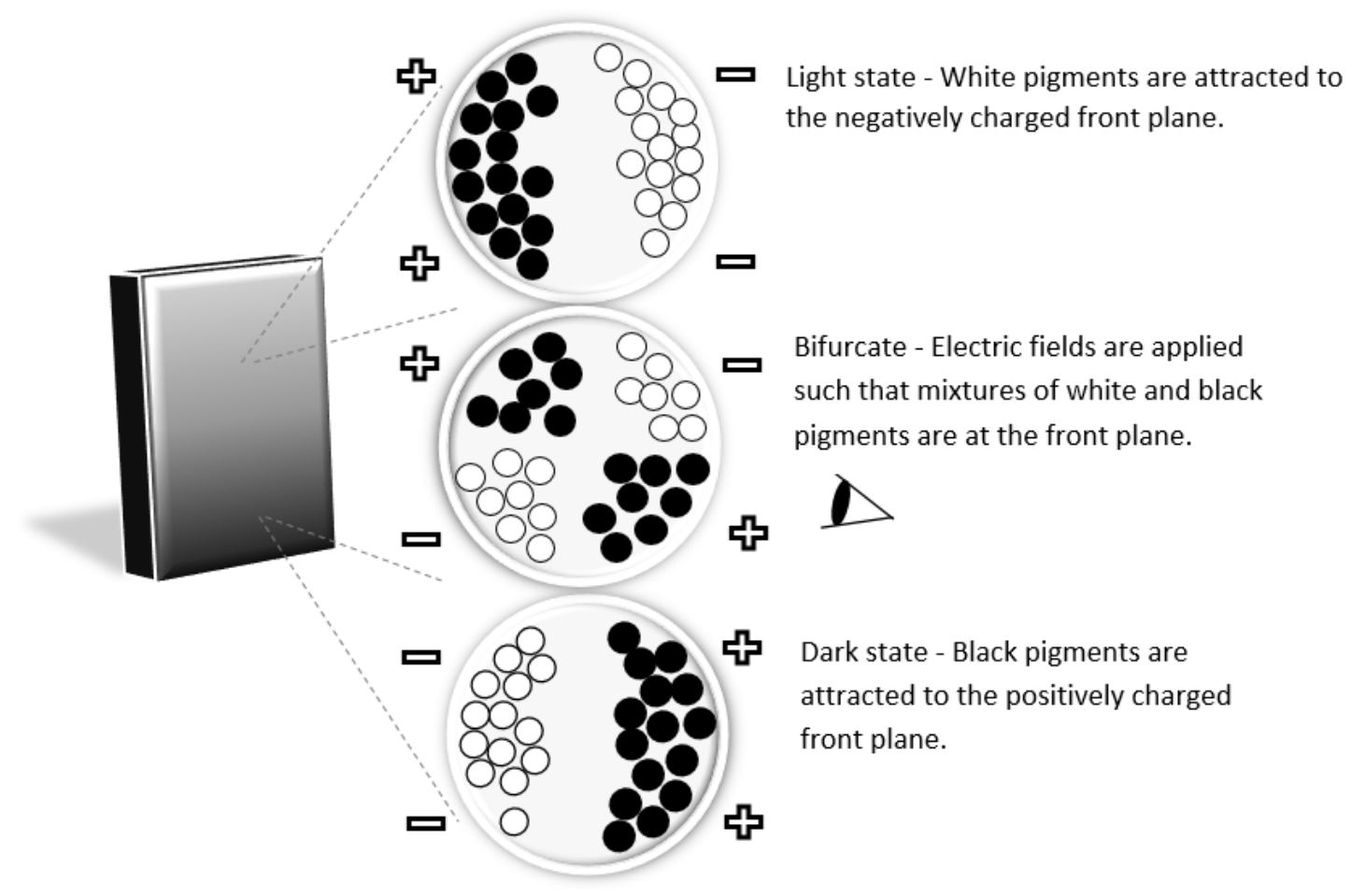

Figure 5: Author's rendition base E-ink microencapsulated electrophoretic technology on [26]. The operating principle of a microencapsulated electrophoretic display.

microencapsulated devices has a downside to image quality. The device has to cycle between light and dark to fully refresh itself for the next frame. If this cycling is not done correctly, the residual of the previous image will remain on the new frame. This problem is commonly referred to as the ghosting effect. In recent years, many researchers have not found a fast and efficient solution to overcome this problem without refreshing the display every time in order to prepare the device for the next frame $[5,3,4]$. Also, the ghosting effect hinders the ability for the display to play video. Device cycling between light and dark takes time, and without cycling, the residual from previous frames remains [31]. 


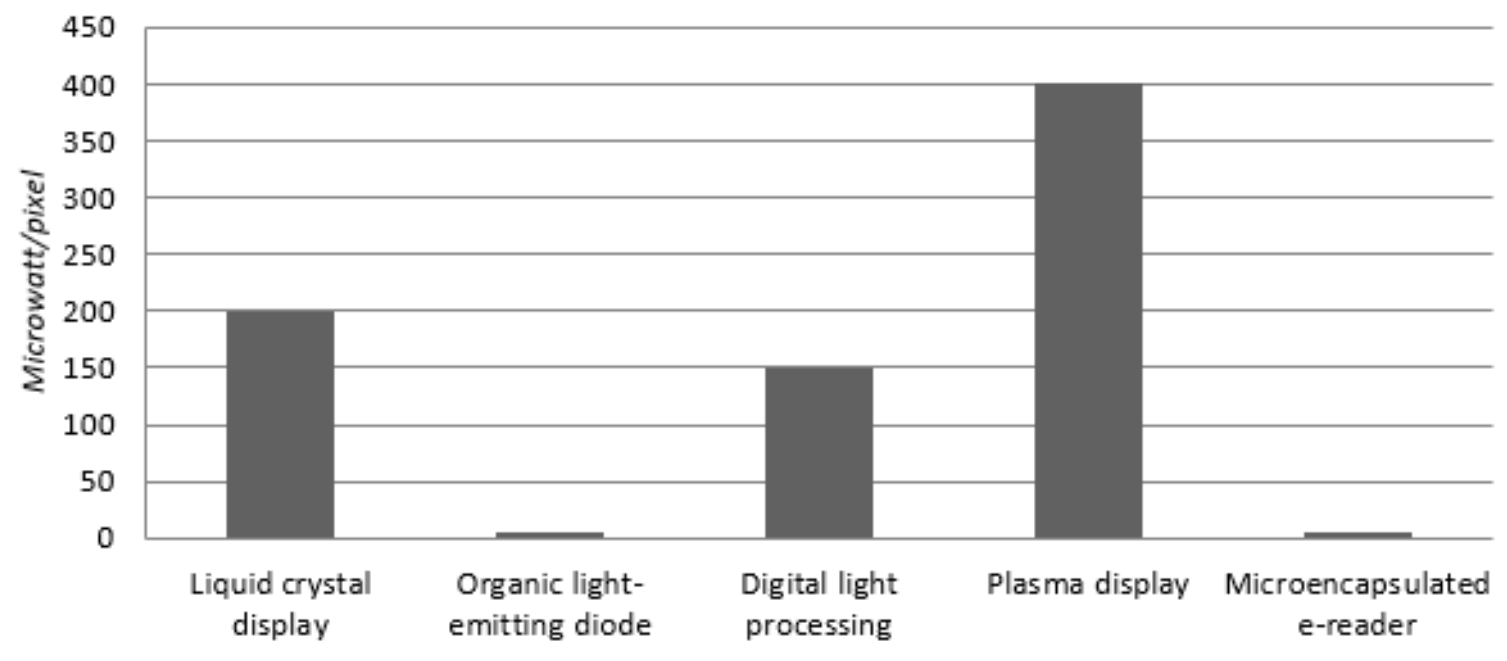

Figure 6: Power consumption comparison among different display technologies. Note that the power indicated for electronic paper display is the power needed to switch between pages, while other displays have to stay on. Values compiled from Display technology overview [25].

\subsection{Alternative electronic display technologies}

Since 1999, microencapsulated electrophoretic display technology has come a long way to make e-readers readily available to consumers. Some developers have been able to introduce colors in microencapsulated devices using multichromatic subpixels on flexible substrates $[32,33,34]$. E Ink is the largest patent holding company for microencapsulated electrophoretic display technology, and many others have invested their effort and money into the technology [26]. The slow response time and the ghosting effect remain the 2 biggest drawbacks.

\subsubsection{Electrowetting display technology}

Electrowetting is one of the most appealing alternative technologies for electronic paper displays. An image is presented by manipulating surface wetting properties of a droplet. Figure 7 shows the principle of an electrowetting display, 
where a strong voltage is applied between the liquid and surface interface.

Researchers have demonstrated a video capable of rapid switching with a response time of 10 milliseconds $[35,36]$.

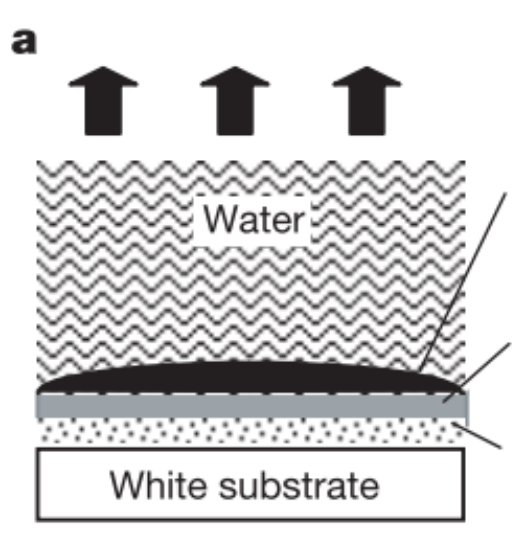

Homogeneous oil film

\section{Coloured oil \\ Hydrophobic \\ insulator \\ Transparent electrode}

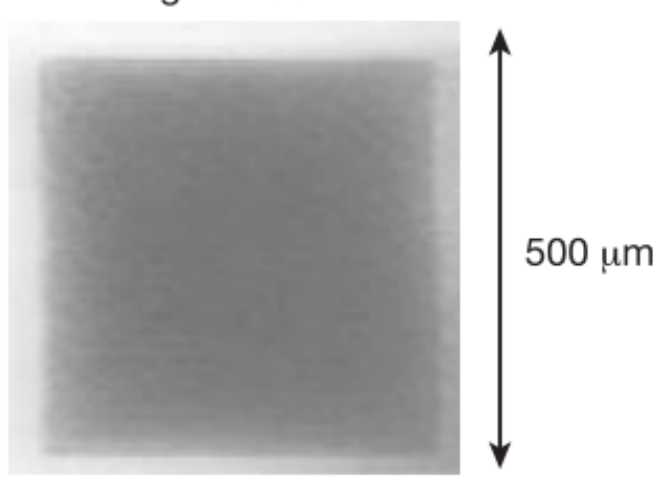

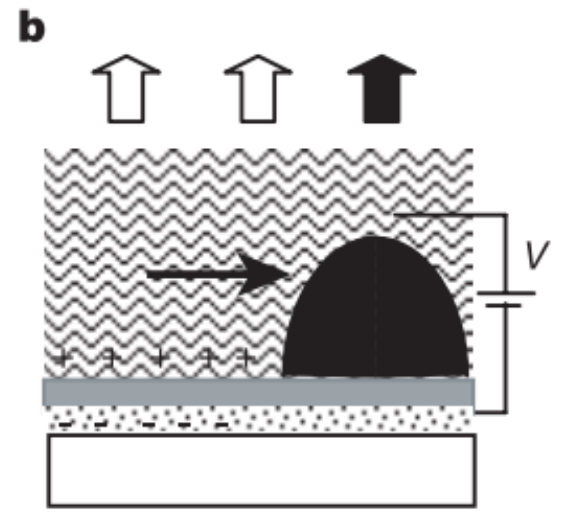

Oil pushed aside

Figure 7: The operating principle of an electrowetting device. (a) Oil is spread out when no voltage is applied. (b) Surface tension between the liquid and solid interface is high; oil contracts when voltage is applied. Reprinted with permission from Macmillan Publishers Ltd: [Nature] ([35]), copyrighted (2003).

In the corner of figure 7, colored oil on top of a hydrophobic insulator with no applied power is shown. At first, the oil is spread out because of low surface tension between two hydrophobic interfaces. However, when the voltage is applied, the surface tension increases as the hydrophobic insulator becomes hydrophilic. Thus, the oil is pushed to the side, and the ink is more concentrated to show a darker 
image. The advantage of electrowetting devices is their fast response time. However, contrast of the electrowetting device is not yet optimized. It is also more difficult to introduce colors in electrowetting devices [37].

\subsubsection{Liquid powder display technology}

Liquid powder display technology was first presented in SID 2003 by a team of researchers at Kyushu University and Bridgestone Corp. [38, 39]. Figure 8 illustrates the composition and cross-sectional view of a liquid powder display. The colorant powders in a liquid powder display are driven up or down within a 50-micron gas gap. The investigators recorded an excellent speed of 0.2 milliseconds for liquid powder display to switch between pixels. Liquid powder displays are also bistable because of electrode polarization and Londonvan der Waals interactions. The challenge that researchers faced regarded the optical performance of the device. The light state of the liquid powder display is not yet optimized, and thus, the contrast is limited. Liquid powder display technology requires roughly 5 times more energy than microencapsulated electrophoretic devices [40].

\subsubsection{Electrochromic display technology}

In the automobile industry, the electrochromic displays are often used to tint rear view mirrors automatically. Electrochromic displays are also used in window glass for users that want privacy, choosing between transparency and translucency. The electrochromic displays use a background color to distinguish between light and dark contrast. Electrochromic displays use color change in chemical reaction. If a reaction for which its reactant and product have different colors, and the direction of the relation can be altered with an external source, then they serve as a potential candidate for the electrochromic display. There are various materials that fit this 


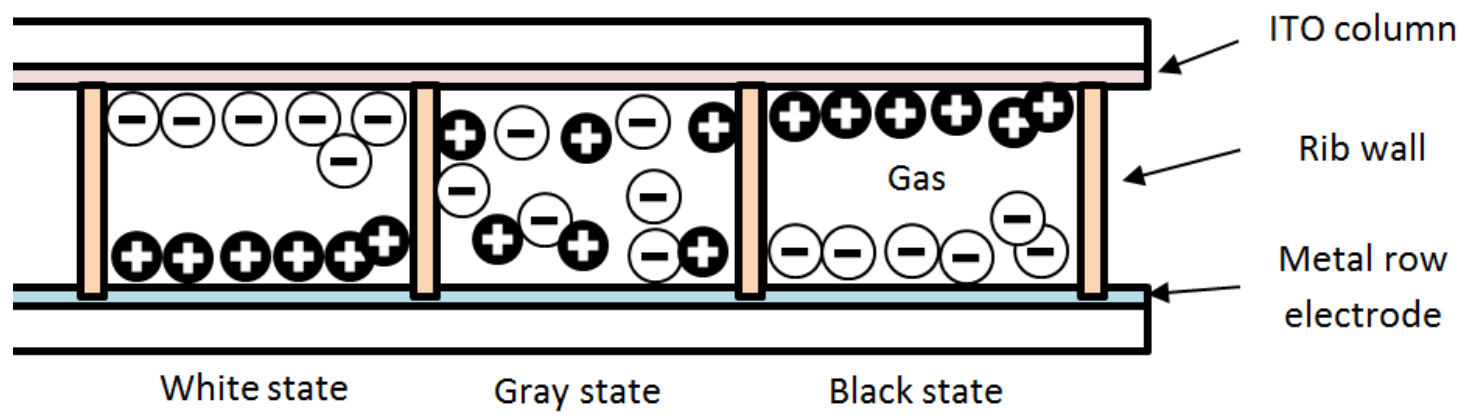

Figure 8: Author's rendition base on Liquid powder display article [40]. Cross section of a liquid powder display switching between states. Permission from John Wiley and Sons: [SID] ([40]), copyrighted (2012).

description, but reactions with the desired color spectrum are not common.

Viologen, which reflects blue when oxidized, can provide superior contrast when used with a white background porous matrix such as $\mathrm{TiO}_{2}[41,42,43]$. Figure 9 shows the crosssection of a electrochromic display with viologen-absorbed $\mathrm{TiO}_{2}$ thin film. The response time of the device depends on the reaction rate. Finding a composition of materials that satisfies the above conditions is difficult. [44, 45] Many researchers are investigating and developing materials that have a fast reaction speed and desired color when undergoing a redox reaction. A group of scientists found that poly(3,4-ethylenedioxythiophene) (PEDOT) and similar compounds can provide a switching rate less than 10 milliseconds in a nanotube-based ultrafast electrochromic display. However, this high switching rate can only be obtained in a $20 \mathrm{~nm}$ thin film, which is too thin for display purposes [46]. 

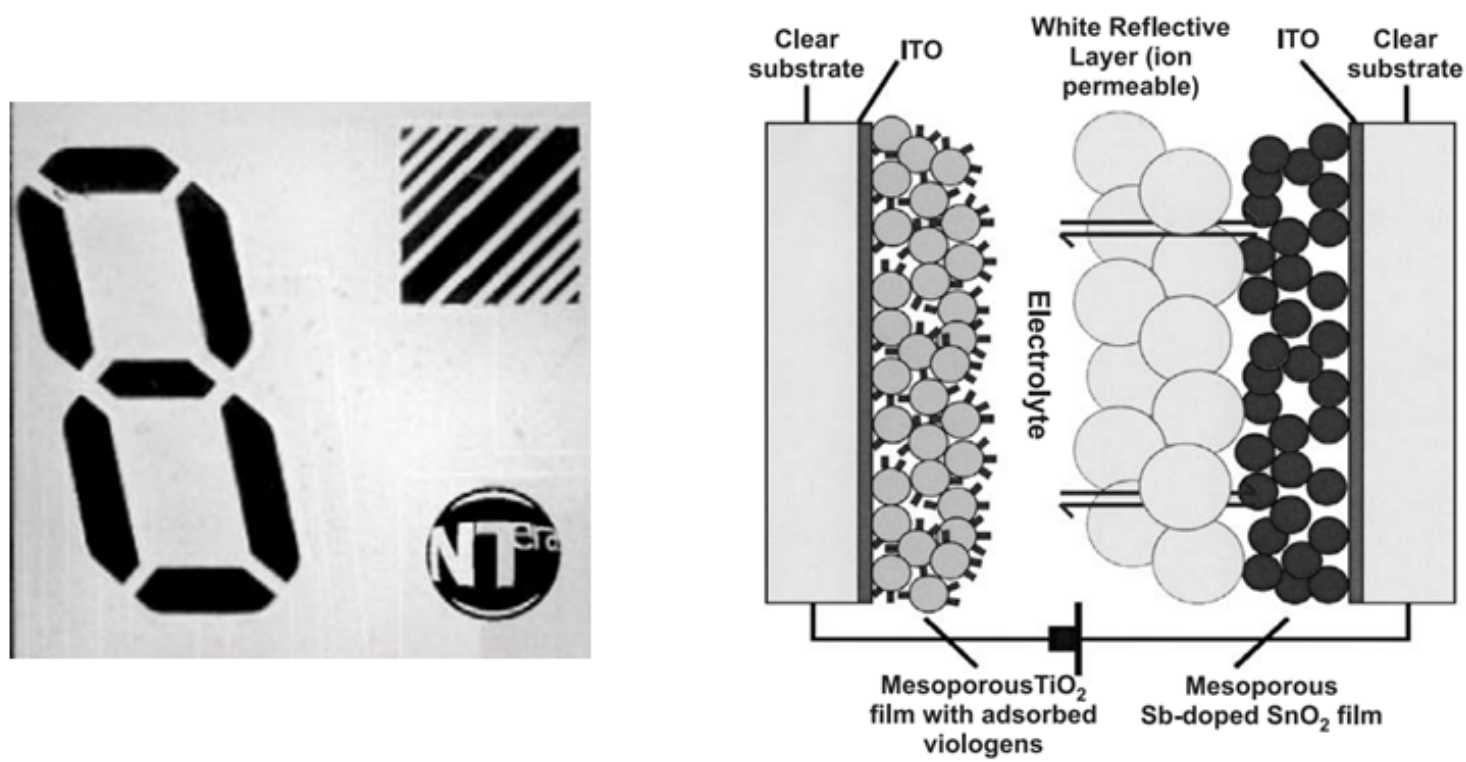

Figure 9: The operating principle of an electrochromic device. Reprinted from Solid State Ionics 165/14, D. Corr, U. Bach, D. Fay, M. Kinsella, C. McAtamney, F. OReilly, S. N. Rao, N. Stobie, Colored electrochromic paper-quality displays based on modified mesoporous electrodes / 316, 2003, with permission from Elsevier.

\subsubsection{Microcup ${ }^{\circledR}$ electrophoretic technology}

Microcup ${ }^{\circledR}$ technology, originally developed by SiPix Technology, Inc., uses roll-to-roll synchronized lithographic manufacturing process that can be used to pattern rectangular cups in an array on a flexible substrate to manufacture flexible electronic paper displays. Similar to microencapsulated electrophoretic devices, the polarized dark and light particles are sealed in these microcups. The profiles of these rectangular cups are shown in figure 10 [47].

These arrays have shown to provide excellent response time, brightness, image uniformity, and longevity with the optimization of particle-particle and particle-environment interactions. Microcup ${ }^{\circledR}$ also provides high temperature stability, and the device can still function even if parts of it are cut or sliced (as shown in figure 11). However, the driving voltage is nearly double that of the 


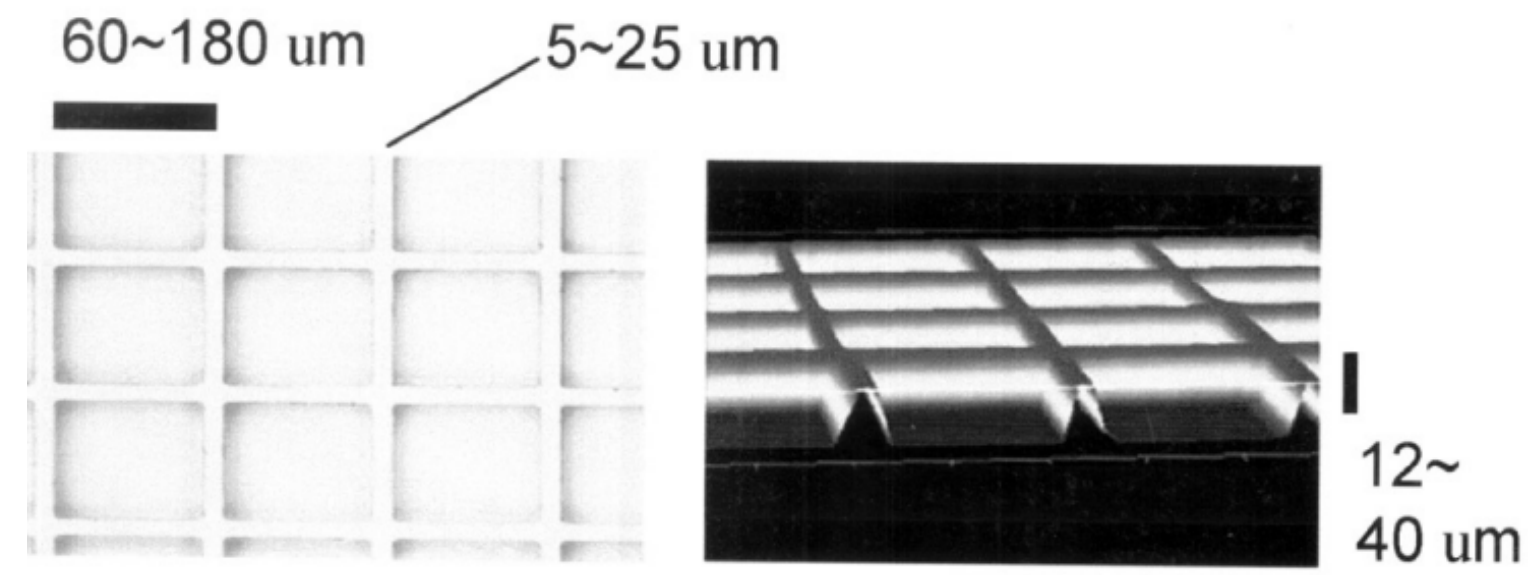

Figure 10: Microcup ${ }^{\circledR}$ profile of SiPix electrophoretic display. Reprinted by permission from John Wiley and Sons: [SID Symposium Digest of Technical Papers] ([48]), copyrighted (2012).

microencapsulated electrophoretic devices [48, 49].
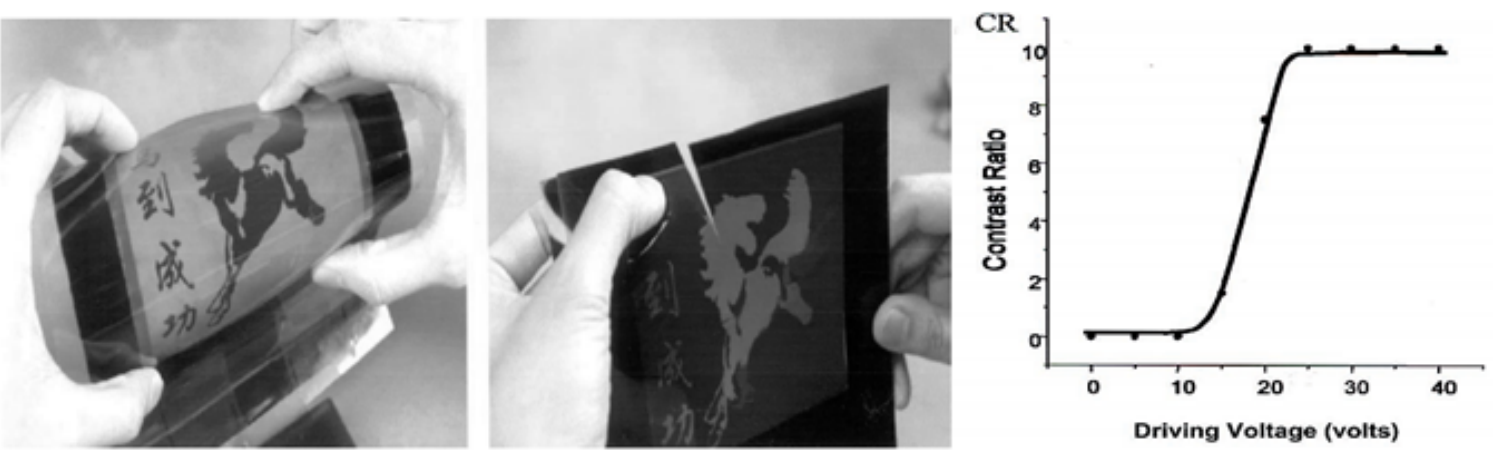

Figure 11: A Microcup ${ }^{\circledR}$ electrophoretic display presenting an image after it is cut. The right shows the driving voltage of Microcup electrophoretic display. Reprinted by permission from John Wiley and Sons: [SID Symposium Digest of Technical Papers] ([48]), copyrighted (2012).

\subsubsection{Electrokinetic display technology}

In 2009 Koch et al, Hewlett-Packard Laboratories (HP Labs) presented their

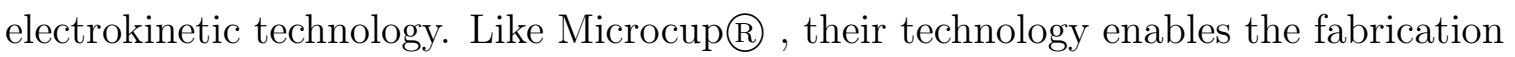


of durable and flexible electronic paper displays [50]. Similar to the ink used in the reverse-emulsion electrophoretic display technology, ink used in electrokinetic display technology also consists of reverse-emulsion nanodroplets. Yeo et al at HP Labs presented a grayscale pixelation with an active-matrix backplane using electrokinetic technology [51]. In 2012, the researchers at HP Labs announced the
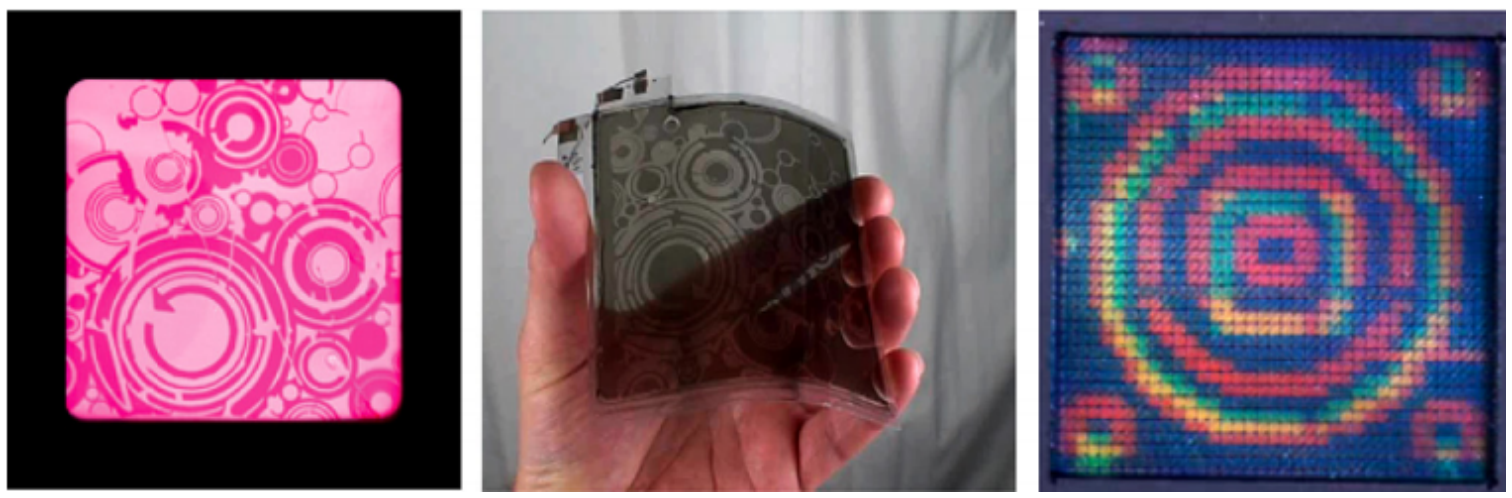

Figure 12: Color electronic paper displays using electrokinetic display technology. Reprinted by permission from Cambridge University Press: [MRS Bulletin Online Proceedings Library] ([51, 52]), copyrighted (2011).

first low-power, paint-like, color reflective display utilizing their electrokinetic display technology. Figure 12 illustrates color electronic paper displays using electrokinetic technology [53].

\subsection{Related research work on nanodroplet electrophoretic devices}

In the context of electronic paper, a complete theoretical modeling of concentrated systems of charged particles dispersed in a liquid medium has been elusive [54]. The physics between electrostatics, particle motion, and transport of diluted species includes particle-particle interactions, particle-electric field interactions, and electric field distortion by a porous medium. Original theories are based on ionic transport, where the ions are modeled as point charges. For this 
study, evaluating the physics within the porous matrix is important. Therefore, this part of the literature review summarizes research on similar systems.

Electrokinetic transport in a porous medium is widely studied. However, systems are usually analyzed in macroscale for civil and chemical engineering. P.J. Barz concluded that the electrokinetic flow velocity of a non-polar liquid traveling through a porous medium increases as the applied voltage increases in Determination of the Zeta Potential of Porous Substrates by Droplet Deflection by Dominik [55]. This study suggests that the response of the particle is proportional to electrostatic force. However, results obtained in macroscale often do not translate directly when the length scale decreases.

Researchers from Belgium conducted a steady-state current test on an electrophoretic image display. The devices tested were similar to the reverse-emulsion electrophoretic display, but without the porous medium. Reverse micelle dissociations in higher voltage environments were observed. Dissociated surfactants lowered the mobility of the ink nanodroplets [56]. A study conducted by the same investigators showed agreement with that of the previous researchers. Both findings saw stronger electrostatic screening of the electric field and a faster decay of transient current in their devices. In a later study, at high voltage, the approximated analytical solution still showed effective electrostatic screening by the high charge density [57]. Their simulation model is based on a previous 1-D numerical model that includes drift, diffusion, generation, recombination, and voltage drop of the electric double layers (EDL) within the planar electrode [58]. This study indicates that above a certain driving voltage, the ink performance will become unstable.

Electrostatic screening at electrodes by the ionic species in fluids is widely studied because of its significance for capacitors [59, 60, 61]. Even in an electrolyte 
environment where the cations are immobilized, the mobilized anions are still capable of screening the electric field that is applied [62]. Original analytical models of electric double layers are based on point charge assumption. There are two drawbacks of point charge assumption. First, when the applied potential is high, point charge assumption will result in the unrealistic accumulation of ion concentration near the electrode. In reality, the actual driving voltage needed for a reverse-emulsion electrophoretic display is too high to model with point charge assumption [63]. Second, even though point charge assumption is reasonable for size orders of magnitude smaller than nanoscale, when the transported species are an order of magnitude larger than angstroms, the steric effect will be significant. For example, in biological science that involves charged nanodroplets in electric double layers, steric effects will have to be included [64]. In chapter 5 of this study, a coarse steric compensation method was implemented to overcome the two drawbacks.

Ink-carrying agents in HP's electrokinetic display technology are also reverse-emulsion nanodroplets [65]. Researchers and developers in Hewlett-Packard were able to use small-angle X-ray scattering to detect several different compositions of micelle core size plus shell size. With dynamic light scattering and transient current measurements, they were able to verify the hydrodynamic radius of their reverse-emulsion. They concluded that increasing polyamines in the surfactant head were able to increase their micelle size. Nanodroplet charge is an important variable that determines the mobility of the particles. Prior investigators of similar nanodroplets measured high-concentration zeta potential in their systems using light scattering technique [66]. Researchers were also able to measure the electrostatic and hydrodynamic forces using optical tweezers [67]. The results measured using optical tweezers were in agreement with the Helmholtz-Smoluchowski and Debye-Hückel models. 


\section{CHAPTER 3}

\section{THEORY}

The content of the theory chapter is divided into three sections. First, the order of magnitude comparisons of cross-discipline forces are studied to determine the main driving force of a reverse-emulsion electrophoretic display system. Second, electrokinetic force, the main driving force of ink nanodroplets in a reverse-emulsion electrophoretic display system is studied. Lastly, the modeling of electric double layers is reviewed with a focus on the Poisson-Nernst-Planck model.

\subsection{Force order of magnitude comparison}

It is important to study the order of magnitude of representative forces between the different interactions to understand the relative importance of interactions within a reverse-emulsion electrophoretic display. Brownian motion is the random molecular motion governed by thermal energy and representative particle length. Brownian force can be interpreted as follows: $O\left(\frac{\mathrm{k} T}{\alpha}\right)$. In this order

of magnitude analysis, $\mathrm{k}$ is Boltzmann's constant, $1.381 X 10^{-23} \frac{\mathrm{J}}{\mathrm{K}} . T$ is temperature, and $\alpha$ is the representative particle length. Isothermic room temperature assumption of the operating device causes Brownian motion to be insignificant. London-van der Waals forces are characterized by the interaction between polar particles in a solution. London-van der Waals forces can be represented by $O\left(\frac{\mathrm{A}_{e f f}}{\alpha}\right)$. In this equation, $\mathrm{A}_{\text {eff }}$ is the Hamaker constant, which relates to the solvent fluid surrounding the particle, the geometry of the particle, and the potential charge-carrying nature of the particles. The assumption of non-polar ink 
nanodroplets is valid based on the spherical morphology of the ink nanodroplets. Therefore, London-van der Waals forces within a reverse-emulsion electrophoretic system are negligible. Hydrodynamic interactions, including the viscous and inertial forces, relate nanodroplets' mobility to the fluid medium and its morphology. The viscous force can be represented by $O(\mu \alpha U)$. In this equation, $\mu$ is the viscosity of the fluid. $U$ is the particle velocity. The inertial force can be characterized with $O\left(\alpha^{2} \rho U^{2}\right)$, where $\rho$ is the density of the fluid. In a microsystem, where the representative length is in microscale and the velocities are low, viscous force prevails. This is shown by the ratio between the inertial and viscous force. The gravitational body force is represented as $O\left(\alpha^{3} \Delta \rho g\right)$, where $\Delta \rho$ is the difference between the density of the particle and fluid. Gravitational body force decreases rapidly as the length scale decreases; thus, it is also negligible in microsystems [68].

\subsection{Electrostatic force and electrophoresis phenomena}

Based on the characteristics and assumptions of a reverse-emulsion electrophoretic display, minor interactions such as Brownian motion, London-van der Waals interactions, and inertial and gravitational interactions are negligible. This leaves the major interactions in electrophoresis, viscous and electrostatic interactions, to be discussed. Electrostatic interactions, including long-range and short-range interactions, are the most dominant interactions in reverse-emulsion electrophoretic devices. Electrostatic phenomena are responsible for the motion of charged species in an electric field. Long range interaction is derived based on Coulomb interaction in a vacuum. From Coulomb's law, the two charges $q_{1}$ and $q_{2}$ in a vacuum exert a force on each other that is inversely proportional to their distance squared $d$, as shown in equation 1 , where $k_{e}$ is the Coulomb's constant. In 
a vacuum, the theoretic force between particles with charges $q_{1}$ and $q_{2}$ decays as the distance between the charges increases. Despite this fact, the theoretic force will never actually reach zero.

$$
F=k_{e} \frac{q_{1} q_{2}}{d^{2}} \quad \text { Equation } 1
$$

This phenomenon plays the same role with respect to the nanodroplets. Long-range interaction is the reason why charged electrodes attract or repel the nanodroplets. Species are in motion until their attraction and repulsion forces become equal and opposite. In reality, solely studying long-range interaction in a reverse-emulsion electrophoretic display is insufficient. As mentioned above, the ink nanodroplets are like dispersed particles in a non-polar organic solvent. The resulting solution is heterogeneous. This means that it contains either or both multiphase and dissimilar characteristics and properties. Interactions that account for particle interaction, solvent interaction, and field interaction are considered short-range interactions. Along with viscous interactions, they are known as the electrophoretic retardation force [69]. Short-range interaction in a dielectric solvent follows $\varepsilon_{r} \varepsilon_{0} \zeta$ where $\varepsilon_{r}$ is the relative permittivity of the fluid, $\varepsilon_{0}$ is the permittivity of free space $8.85 \times 10^{-12} \frac{C}{V} m$, and $\zeta$ is the electrokinetic potential of the particles. Figure 13 shows a visualization of long-range, short-range, and viscous forces acting on an ink nanodroplet and its resulting direction.

The most widely used model for charged species mobility, $\mu_{m}$, in an electrophoretic system that has taken both viscous and electrostatic interactions into consideration was developed in 1903 by Smoluchowski [70]. Equation 2 shows that the mobility of the transporting species is greatly influenced by the electrostatic potential of the particles and the dynamic viscosity of the fluid medium. When the 


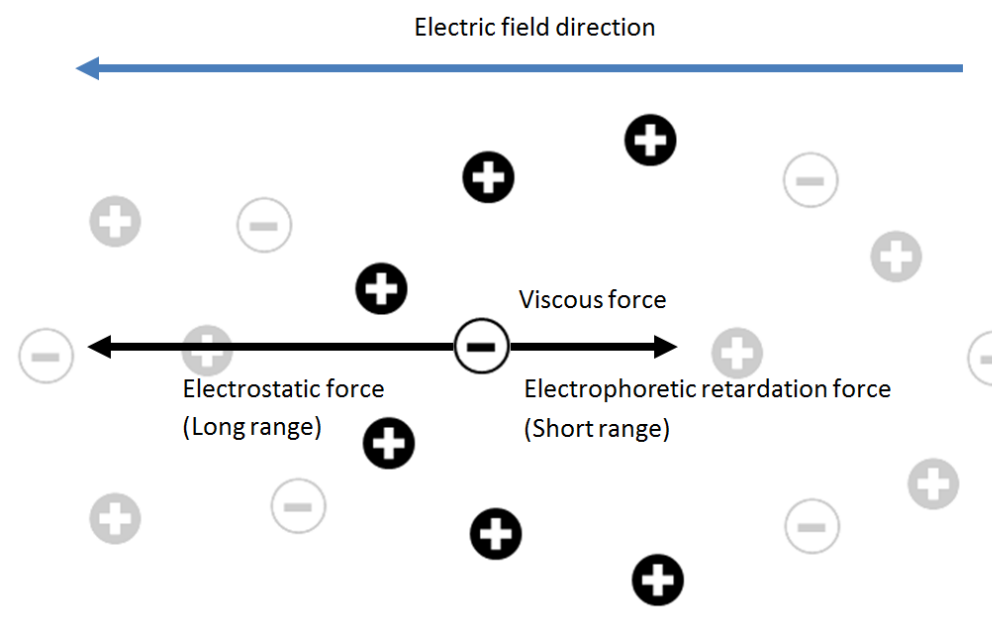

Figure 13: Visualization of the forces acting on an ink nanodroplet in an ionic solution.

electrostatic potential of a particle increases, the mobility of the particle increases; when the viscosity of the system increases, the mobility of the system decreases.

$$
\mu_{m}=\frac{\varepsilon_{r} \varepsilon_{0} \zeta}{\eta}
$$

Equation 2 reveals just the mobility of ink nanodroplets in a reverse-emulsion electrophoretic system. To gain a broader understanding, equations modeling the electric double layers are reviewed in the next section.

\subsection{Electric double layers}

Electrostatic screening, not to be confused with the shielding effect in electron shells, is a phenomenon of the electric double layers $[71,72,73,74,75]$. The electric double layers consist of a compact counterion layer (for which this layer forms a high charge density opposite to the electrode), followed by a diffuse layer at the interracial surface of the charged electrode. The high charge density opposite to the electrode acts as a barrier for which it screens the applied potential on the 
electrode. Effective potential in the diffuse layer is low or close to being nonexistent. Ink nanodroplets that are in the diffuse layer are free to migrate, disperse, and diffuse into the porous medium. A schematic representing electric double layers in equilibrium with equal and opposite charge is shown in figure 14.

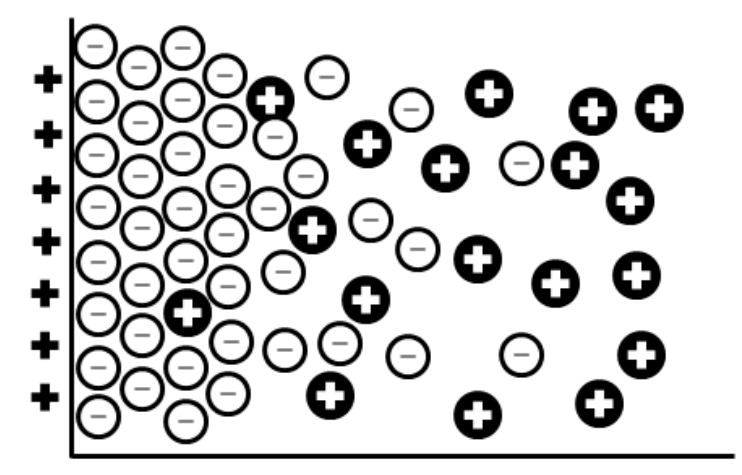

Distance from electrode

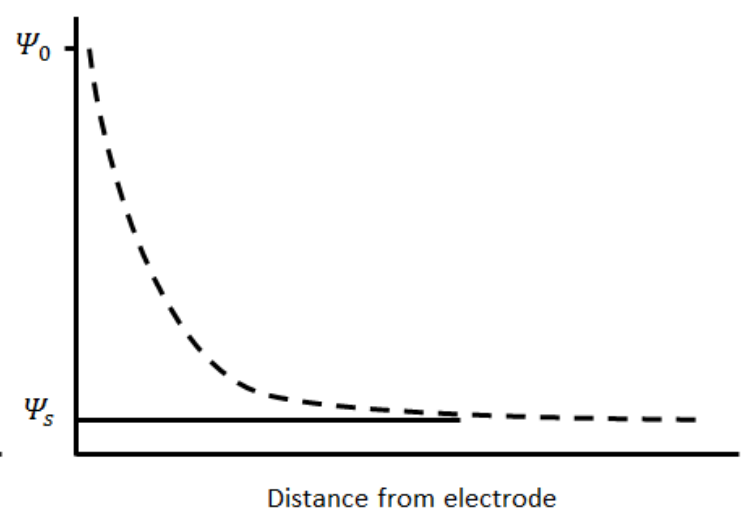

Figure 14: Visualization of electric double layers with positively and negatively charged particles and the resulting internal potential near an electrode.

$\Psi_{0}$ is the applied potential at the electrode, while $\Psi_{s}$ is the effective potential at the outer diffuse layer. Electrostatic screening is illustrated by the exponential decay of the electric potential within the device. It is very different from what is expected in a non-electrolytic solution or metallic interface where the electric potential between the two planes decreases linearly. Study of charged interfaces started in the 1800s [76]. The first experimentally confirmed theory was formulated by Walther Nernst. The Nernst equation observes the conservation of matter, conservation of charge, and conservation of energy [77, 78]. The first model for electric double layers was formulated by Helmholtz [79, 80]. The theory by Helmholtz accounted for the differential capacitance of the Helmholtz layer, the dense layer of counterions attracted by the charging interface, and the capacitance due to a metal interface. In the early 1900s, Gouy and Chapman, who worked 
independently, were credited for their modeling of the electric double layers known as the Gouy-Chapman theory. Capacitance alteration due to solvent dipoles and diffuse layer are considered in the Gouy-Chapman theory [81, 82]. Combining the two theories developed by Gouy-Chapman and Helmholtz, German physicist and Nobel laureate Otto Stern developed an improved version known as the Stern layer. The Stern layer accounted for the capacitance of the charging interface, metal interface, solvent dipoles, and diffuse layer capacitance [83]. Poisson-Boltzmann equations are widely used in steady-state electric double layers modeling $[84,85,86]$. Poisson-Boltzmann equations are known to represent the Gouy-Chapman theory in electrochemistry [87, 88], the Debye-Hückel theory in solution chemistry [89], the Derjaguin-Landau-Verwey-Overbeek (DLVO) theory in colloid chemistry [90, 91], and the Poisson-Boltzmann theory in biophysics [92, 93]. To use the Poisson-Boltzmann equations, thermodynamic equilibrium and ionic distributions that are not affected by fluid flows have to be assumed. The last assumption is inadequate for more complex scenarios (including this paper). Poisson-Nernst-Planck equations are more complex and computationally intensive, but they are widely used in situations where the electroosmotic flow is through rapidly changing dimensions or narrow microchannels [94, 95, 96, 97, 98]. The rapid advancement in computational techniques in recent years have allowed more complicated electric double layers models to be developed and examined. For example, the interactions between the transported species and their size accommodation are studied with size-modified Poisson-Boltzmann and size-modified Poisson-Nernst-Planck equations [99, 100, 101]. However, limitations of these models arise when the electrical voltage applied is no longer in microscale. This was also observed in the simulation section where the model fails to converge because of the unrealistic concentration accumulation at the charging surface when the applied 
voltage is too high.

\subsection{Poisson-Nernst-Planck modeling}

Under an applied electric field, the concentration of these nanodroplets near the positively charged electrode will increase until steady-state is reached. An electrostatic screening then occurs, by which the locally high concentration of negatively charged nanodroplets near the electrode repels other nanodroplets from the bulk solution. One can visualize it as a competition between the electric field (pulling the opposite charged nanodroplets toward the charged electrode) and the concentration of the charges (the dense charges build up via the high concentration of nanodroplets). If the concentration of nanodroplets is high enough, the electric field is screened heavily, and the electric field in the middle layer approaches to zero. On the other hand, if the electric field is strong and there are not enough nanodroplets to screen the electric field completely, the charge density of the ink nanodroplets near the repelling electrode approaches zero [57]. The electrostatic screening phenomena for charged particles can be described by the steady-state Poisson-Nernst-Planck equations that have been well established for modeling ionic species in biochemistry and electrochemistry $[68,102,103,96]$. The Poisson-Nernst-Planck equations consist of the Poisson equation and the Nernst-Planck equation. The Poisson equation is shown below in equation 3 , and it describes the electrostatic potential in the ionic solution, where $\varphi$ is the electrostatic potential, $\varepsilon_{0}$ is the permittivity of vacuum, $\varepsilon_{r}$ is the relative permittivity of the solution, $n_{i}$ is the reference density of ionic species $i, Z_{i}$ is the charge number of the ions in the solution, and $e$ is the elementary charge. 


$$
\nabla^{2} \varphi=-\frac{1}{\varepsilon_{0} \varepsilon_{r}} \sum_{i} Z_{i} e n_{i}
$$

Equation 3

The Nernst-Planck equation is shown below in equation 4 , and it describes the transport of ionic species in a fluid medium. The flux of the ionic species $J_{i}$ depends on variables also seen in the Poisson equation, as well as on diffusivity $D_{i}$ of the ionic species, the Boltzmann constant $k$, and absolute temperature $T$. The Nernst-Planck equation accounts for diffusive flux because of an electric field, thermal energy, and ionic migration in an electric field [104, 105].

$$
J_{i}=-D_{i}\left(\nabla n_{i}+\frac{z_{i} e n_{i}}{k T} \nabla \varphi\right) \quad \text { Equation } 4
$$

The diffusivity of the ionic species is estimated based on the Stokes-Einstein equation shown below in equation $5[106,107]$, where $R$ is the gas constant, $T$ is absolute temperature, $\mathrm{N}_{\mathrm{A}}$ is Avogadro's number, $\mu$ is dynamic viscosity, and $r$ is the nanodroplet radius. For example, the Stokes-Einstein equation can be applied to particles with a $90 \mathrm{~nm}$ radius at room temperature, and with a dynamic viscosity of $2.012 \mathrm{cP}$ for the organic solvent tetralin (1,2,3,4-tetrahydronaphthalene) [108]. Given these constraints, the calculated diffusivity would be $D=1.2 \times 10^{-12} \mathrm{~m}^{2} / \mathrm{s}$.

$$
D=\frac{R T}{N_{A}} \frac{1}{6 \pi \mu r}
$$

The diffusivity term influences the mobility of transported species in a reverse-emulsion electrophoretic display. For transient analyses, diffusivity of the species can be refined by adding influential terms in simulation to achieve a more accurate switching response. However, contrast of a reverse-emulsion electrophoretic display at steady state does not have a direct correlation with diffusivity or mobility. As mentioned above, the Poisson-Nernst-Planck model of the electric 
double layers couples nanodroplet concentration and electric potential within the device at steady state. However, the Poisson-Nernst-Planck model does not account for the steric effect - that size relevance to space occupied [109, 110, 63]. The concentration of ink nanodroplets is (unrealistically) free to build up at the electrode without bounds. This limits the usefulness of the Poisson-Nernst-Planck equations for predicting saturation thresholds. The steric effect has to be included, and this necessitates the characterization of the ink, beginning with nanodroplet size, as presented in chapter 4. 


\section{CHAPTER 4}

\section{INK CHARACTERIZATION}

\subsection{Nanodroplet ink concentration}

The most important parameter for this investigation is the concentration of nanodroplets in the tetralin solvent. The concentration is estimated based on the weight of dried material, solution density, and assumption of spherical nanodroplet geometry. The amount of dry material used to synthesize the ink is proportional to the number of nanodroplets formed. Knowing the mass of dry material and nanodroplet size, the concentration of the ink can be estimated based on volume fraction. The batch R129 used for this study had an estimated concentration of $1.40 \times 10^{-4} \frac{\mathrm{mol}}{\mathrm{m}^{3}}$. Dilutions were made from this $100 \%$ concentrated sample by adding more solvent.

\subsection{Nanodroplet charge estimation}

Typically, in pure non-polar solvents, the total charge of nanodroplets are only a few elementary charges $[111,112]$. After introducing surfactants, the nanodroplets are stabilized to the charge of either $+\mathrm{e}$ or $-\mathrm{e}[113,114,115]$.

\subsection{Nanodroplet size measurement and morphology}

Although beyond the immediate study of concentration effects in this investigation, nanodroplet size and morphology are important because size and morphology may influence other factors, such as bistability and spatial uniformity, 
in an assembled display (e.g. using a bonded porous matrix, as in A Thin Porous Substrate Using Bonded Particles for Reverse-emulsion Electrophoretic Displays [13]). Structurally, the nanodroplets are reverse micelles, which form different shapes based on the environment, composition, and stabilization [65]. The morphology of the reverse micelles affects transport and retention behaviors in the porous matrix. Transmission electron microscopy (TEM) has a resolution as fine as 0.47 Aand has been used to image reverse micelles $[116,117]$. Researchers have recorded up to six different types of stable morphologies for reverse micelles by TEM $[118,119,120,121]$. Atomic force microscopy (AFM) can also be used to image reverse-micelles with sub-nanometer resolution [122, 123, 124]. However, AFM requires the use of dry samples. Researchers successfully imaged reverse-micelle with AFM and concluded that structure of the micellar aggregation is strongly influenced by the surface conditions [125]. Other techniques such as flow cytometry, dynamic light scattering, and small-angle x-ray scattering can measure the size of the ink nanodroplets in liquid; however, these techniques cannot provide a visual image. Flow cytometry is widely used in life sciences to analyze multiple characteristics of a sample, where the sample passes through an extremely tube, aided further by hydrodynamic focusing. Samples are forced to pass through the inspection point with narrow confinement. Laser imaging is used to scan the profile of the objects passing by the inspection point. This idea was first introduced in 1953, described as a flow particle separator [126]. Modern flow cytometry instruments can examine heterogeneous solutions and report characteristics such as size and structure [127]. The particle detection range is typically between $1 \mu \mathrm{m}$ and $15 \mu \mathrm{m}$. Subnanometer resolution can be achieved with specialized systems. However, the compatibility of the instrument is also an issue. Usually, flow cytometers are calibrated in an aqueous environment for biological systems but have 
also been used in non-aqueous environments [128, 129]. Dynamic light scattering (DLS) is a fast and accurate tool used to characterize both emulsions and particles. When emulsions and particles disperse or dissolve in a liquid, random particle motion enables a laser to trace the profile of the specie within the sample. Dynamic light scattering is particularly advantageous for soft materials, because the emulsion and particle can stay in the aqueous/non-aqueous state $[130,131,132,133]$. The resolution of a modern DLS instrument can measure a size smaller than 1 nanometer, and the required sample volume can be as small as 2 microliters. The particular instrument that was available for this study is the Zetasizer Nano ZS (Malvern Instruments Ltd., Worcestershire, UK). This instrument is capable of measurement ranging from 0.3 nanometer to 100 microns in diameter. The measurement type includes particle size, molecular size, and zeta potential [134].

\subsection{Atomic force microscopy}

The size and morphology of the micelles were measured by atomic force microscopy. Specimens were prepared by diluting the most concentrated ink with the solvent tetralin. Diluted ink was dispensed onto a glass substrate with a pipette. The sample was then dried at $50^{\circ} \mathrm{C}$ for 2 hours to evaporate the solvent. The remaining solvent was then spin-dried for 5 minutes. Spin-drying helps centrifuge the rest of the solvent, leaving a thin layer of reverse micelles on the substrate. A nondestructive temperature of $50^{\circ} \mathrm{C}$ was chosen to evaporate the solvent slowly. The samples were then placed on the measurement stage in the atomic force microscope. Scans were performed in (non-contact) tapping mode for $2 \mathrm{~m} x 2 \mathrm{~m}$ scan fields. Nanodroplet diameters were estimated from atomic force microscopy data using open-source surface probe analysis software (Gwyddion, Czech Metrology 
Institute, Czech Republic). Figure 15 shows a representative scan, revealing discrete entities with a lateral dimension of approximately 100 nanometers and a maximum thickness of $20 \mathrm{~nm}$. The vertical resolution of the probe is 1 nanometer. The glass substrate had a root-mean-square (RMS) surface roughness below $3 \mathrm{~nm}$. Dilution of

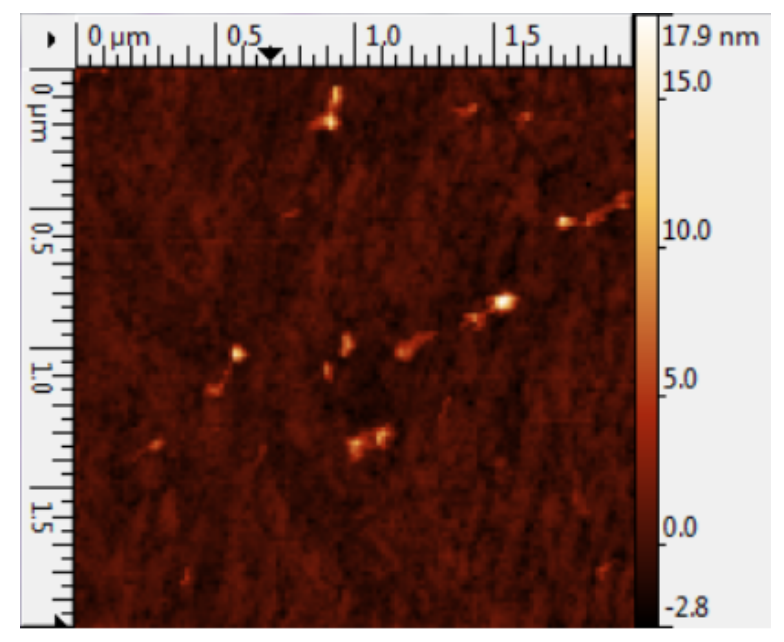

Figure 15: Atomic force microscopy normal incident view of the dried nanodroplets.

the ink to $1 / 800$ th of its full concentration provided the best results when compared with all the other samples. Figure 16, showing the peak area in white, is a 3-D atomic force microscopy image at this level of dilution. For this particular image, there are 3-D features in the z-direction that are distinct from the glass substrate surface profile. These features are approximately $20 \mathrm{~nm}$ in height above the substrates, and most have lateral dimensions on the order of $100 \mathrm{~nm}$.

\subsection{Dynamic light scattering}

Dynamic light scattering was used to quantify the hydrodynamic radius and size distribution of the ink nanodroplets. The output from Zetasizer Nano ZS (Malvern Instruments, Ltd., Worcestershire, UK) indicates that the nanodroplet radius has a single peak at $176 \mathrm{~nm}$. The narrow size distribution from the dynamic 


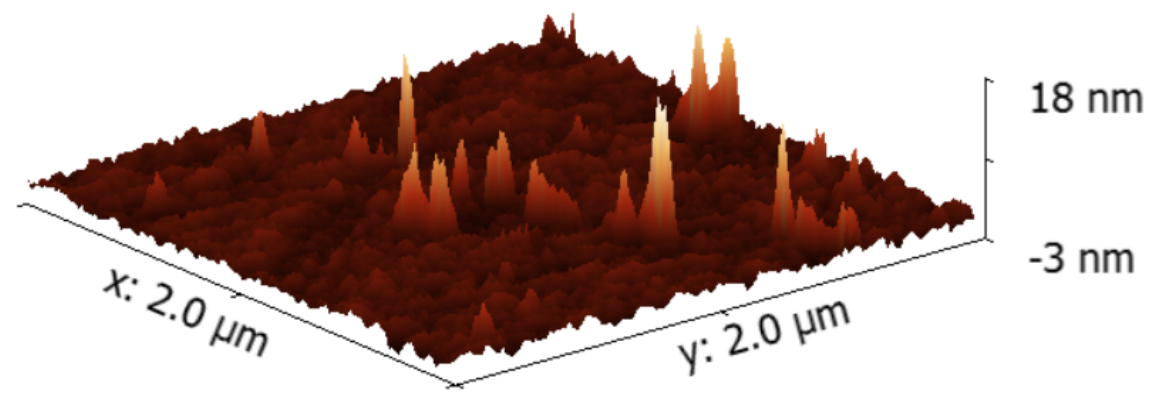

Figure 16: Atomic force microscopy oblique view of the dried reverse micelles.

light scattering result also shows that the nanodroplet is stable.

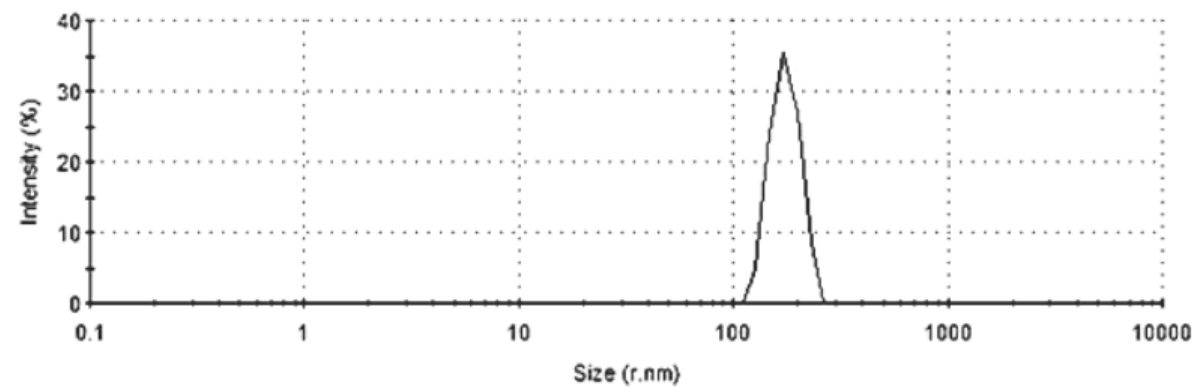

Figure 17: Nanodroplet size distribution, measured by dynamic light scattering. Image courtesy of W. Schulkins.

The size of the ink nanodroplets determined by DLS is in agreement with results measured by AFM. The nanodroplet atomic force microscopy image with the dry sample is flatter than that of the wet sample. The atomic force microscopy values are about half the size of dynamic light scattering, which is not surprising since the atomic force microscopy samples are dry.

4.6 Ink material properties

To model behavior for simulation, material properties of the ink need to be determined. The solvent used for the ink is tetralin. Tetralin 
(1,2,3,4-tetrahydronaphthalene) is a hydrocarbon transparent non-polar liquid with the chemical formula $\mathrm{C}_{10} \mathrm{H}_{12}$. It has a viscosity of $1.968 \times 10^{-3} \mathrm{Ns} / \mathrm{m}^{2}$ at $298.15 \mathrm{~K}$ [135]. The relative permittivity of tetralin at $23^{\circ} \mathrm{C}$ is 2.77 , and the electrical conductivity is $1.35 \times 10^{-8} \frac{S}{m}[136,137]$. A highly diluted ink would have properties similar to its solvent. The following subsection describes the experimental approach that was used to determine the material properties of the ink.

\subsubsection{Ink permittivity measurement}

In an electrophoretic system, relative permittivity, $\varepsilon_{r}$, influences particle mobility, as shown in equation 2. A test device consisting of the ink was prepared between two ITO-coated glass electrodes. A few glass microspheres were inserted into the gap in order to ensure uniform spacing of $10 \mu \mathrm{m}$. This test device acted like a capacitor, placed in parallel with a resistance, $R$, and the cutoff frequency was noted where the output power is one-half of the input power. Resistance was measured in direct current mode and factored out of the resistance-capacitance time constant (in alternating current mode) to reveal the capacitance based on equation 6. Relative permittivity was determined from the capacitance of the device, as shown in equation 7 , where $\varepsilon_{0}$ is the permittivity of the vacuum, $A$ is the plate area, and $d$ is the spacing between plates. Using such a method, the relative permittivity of the ink was determined to have a value of $\varepsilon_{r}=3$. As expected, this value is close to the reported value of 2.77 for the solvent tetralin [138]. The method to determine the relative permittivity is summarized below.

$$
f_{c}=\frac{1}{2 \pi R C}
$$

Equation 6

The cutoff frequency was measured at $97 \mathrm{kHz}$, following equation 6 , to reveal the capacitance of the device. After the value of capacitance was found, the parallel 
plate capacitor model shown in equation 7 was used to determine the relative permittivity.

$$
C=\varepsilon_{0} \varepsilon_{r} \frac{A}{d}
$$

The resulting solution with nanodroplets had higher permittivity than the pure solvent tetralin, and can be explained by the higher relative permittivity of the nanodroplet solute.

\subsubsection{Ink conductivity measurement}

The conductivity of the ink was determined by experimentation of an RC circuit with a test device. Since the internal resistance is known, the relationship between resistance and resistivity is shown in equation 8, where the conductivity is the reciprocal of resistivity, $\rho_{r}$ is the resistivity, $R_{r}$, is the electrical resistance, $A_{s}$ is the cross-sectional area, and $l$ is the material length.

$$
\rho_{r}=R_{r} \frac{A_{s}}{l}
$$

Ohm's law, $V=I R$, was applied to measure the resistance in a reverse-emulsion electrophoretic display cell. DC voltage ranging from $1 \mathrm{~V}$ to $15 \mathrm{~V}$ are applied to the two electrodes, while a multimeter measures the current passing through the circuit. The result is plotted with potential on the x-axis and current as the y-axis in figure 18. By Ohm's law, the slope is the resistance of the cell.

\subsection{Porous medium structure}

The structure of the porous medium is imaged by scanning electron micrsocopy. The porous medium is prepared using $\mathrm{TiO}_{2}$ powder. Figure 19 shows 


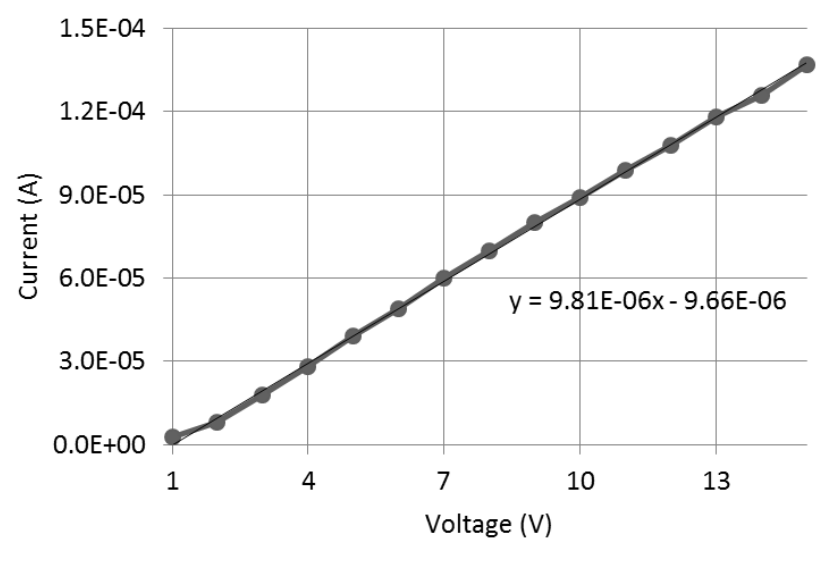

Figure 18: Current-voltage plot for a reverse-emulsion electrophoretic display.

the scanning electron microscopy images of the paste vs. a bounded $\mathrm{TiO}_{2}$ porous medium.
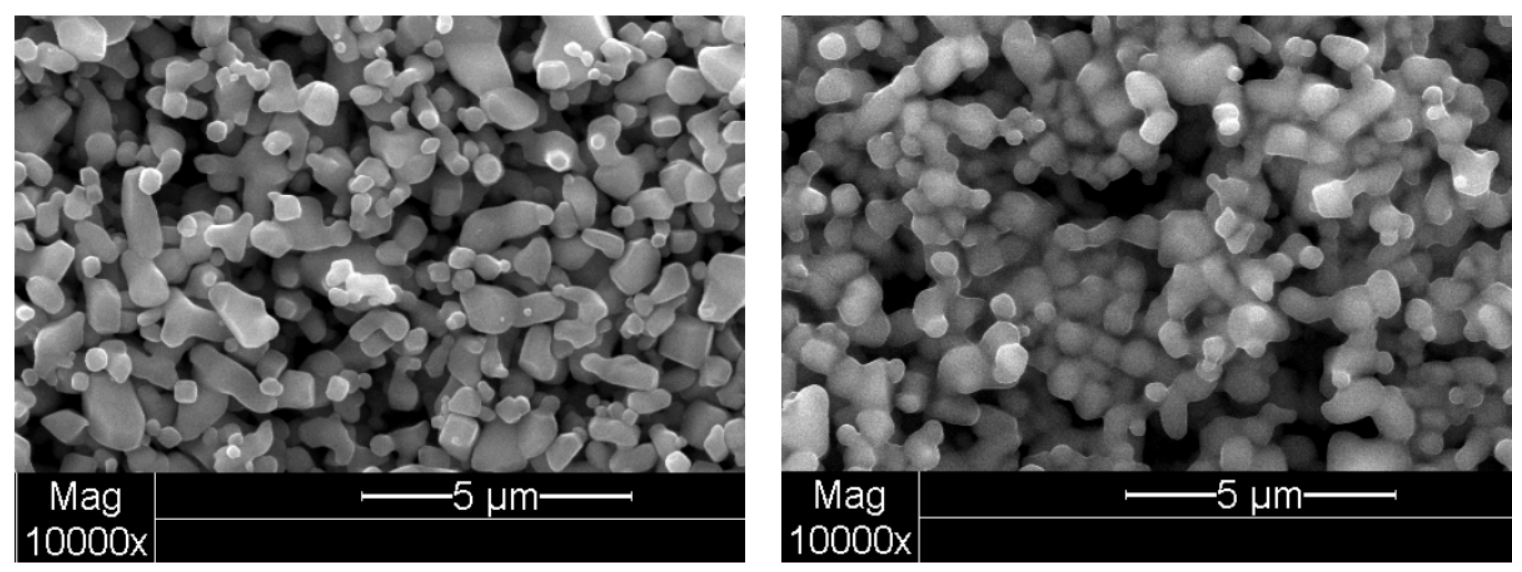

Figure 19: Surface layer comparison of a dry powder (left), and an adhesive bonded substrate (right) with $\mathrm{TiO}_{2}$ powder [13].

These scanning electron microscopy image in figure 19 show that the powder specimen has a rougher structure, whereas the bounded specimen has fillers (adhesives) between particles. Prior studies [13] determined that using a micron-size $\mathrm{TiO}_{2}$ powder bonded with $10 \%$ adhesive can produce a display and with the most consistent layer with the most uniform opacity. The comparison study of bonded 
versus paste (no adhesive) layers have shown that the $25 \%$ paste devices performed with excellent contrast initially, but after long cycles, the display suffers degradation. On the other hand, $25 \%$ bonded display has consistent contrast after cycling; however, the peak contrast is not as good as the paste. Higher volume fractions of $\mathrm{TiO}_{2} 40 \%$ paste showed a promising consistency after cycling; however, the peak dark state performance is between $25 \%$ paste and $25 \%$ bonded display. For this study, the experimental cycling time of each device does not exceed 5 minutes. Accordingly, the $40 \% \mathrm{TiO}_{2}$ paste device that delivers uniformity and good contrast is chosen to be used in this investigation. 


\section{CHAPTER 5}

\section{NUMERICAL SIMULATION}

In the first few sets of simulations, Poisson-Nernst-Planck equations were used to model the device's electric double layers at equilibrium. The equations are applied to simulate $10 \mu \mathrm{m}$ depth electric plates in planar configurations with nanodroplet ink in 1-D simulation. Bulk nanodroplet concentration and its effect on the display contrast performance are addressed. Steric effect is then compensated externally by finding the saturation threshold for the device. A 2-D porous structure built with hexagonal outlines is used to form a uniform microchannel domain for fluid and ink nanodroplets to travel through. A representation of a 2-D hexagonal unit cell is shown in section 5.3. The 2-D geometry is used to compare the difference between the 1-D and 2-D simulations. Parametric simulations with particle tracing provide a visual idealization of how nanodroplet ink moves in the 2-D hexagonal porous structures. Lastly, the transient analyses run in both 1-D and 2-D simulations provide insight into porous medium geometry complications. In order to run the simulation, a few assumptions were made. The first assumption is that nanodroplets behave as a point charge. Second, ink contains two species of nanodroplets, the positive and the negative 1 elementary charge species, equal in concentration and opposite in charge. Third, the ink nanodroplets are spherical and without dipoles. Lastly, the ink nanodroplets are stable and operate at an isothermal operating temperature. 


\subsection{Model setup and information}

The Poisson-Nernst-Planck equations for ion transport is a continuum model widely used in theoretical and computational studies where electrostatic interaction with ion flow is examined. The boundary conditions of the model are shown in figure 20. In multiphysics simulations, time step can also affect the outcome since each time step is computed based on the previous time step. COMSOL Multiphysics (COMSOL, Inc., Natick, Massachusetts, USA) uses the computed values from the previous step, starting from initial boundary conditions to calculate the next step. The convergence tolerance of the model is set at $1 \times 10^{-6}$. The initial condition of each model is specified to be at $0 \mathrm{~V}$ between the two electrodes and when the device is powered up. The voltage is applied at the left end of the model electrode, whereas the right electrode is kept at ground. The concentration of the positive and negative species were set to equal half of the bulk concentration. Initially, the concentration of both species were uniformly dispersed within the models. Over variables such as dynamic viscosity, relative permittivity, charge number, size of the ink nanodroplets, and temperature were kept constant throughout the simulation.

\subsection{Effect of nanodroplet ink concentration on display contrast}

The applied potential on the electrode is assumed to be uniform, and the model of the electric double layers can be simplified to a 1-D simulation. This simulation of the electric double layers using the Poisson-Nernst-Planck model (with an equal charge ratio between $+/$ - ions) does exhibit the anticipated electrostatic screening by the ink nanodroplets. The type of simulation applied here can be described as dilute species because the model is confined to point charges (i.e., disregarding size dimensions). The 1-D simulation of the device with the 
Poisson-Nernst-Planck equation includes the electrokinetic charged particle migration, diffusion via electric field, and electrostatic screening by the charged particles. The diffusion term in the Poisson-Nernst-Planck equation can be modified for specific models to represent different scenarios. The diffusion coefficient in this study is based on the Stokes-Einstein equation because it is suitable for modeling transport of charged spherical particles through liquid. For steady-state analyses, the diffusion coefficient plays a lesser role. However, in a transient analysis where the response speed of the display is desired, picking the right model for the diffusion coefficient is important.

The simulation is specifically used to investigate how the bulk level of nanodroplet concentration in the ink affects nanodroplet concentration near the viewing plane, resulting in electrostatic screening. The modeling parameters can be found in figure 20. For higher precision, the mesh for all models was generated with an element length less than $0.02 \mathrm{~nm}$.

Three simulation outputs were generated: one showed the nanodroplet and repulsive ion concentration at the viewing region, another one displayed the electric potential profile near the viewing region, and the last one compared different levels of bulk concentration when the device is set to either a dark or light state. Steric correction is added to account for nanodroplet saturation in the diffuse layer. The simulated levels of bulk concentration range from $1.74 \times 10^{-5} \mathrm{~mol} / \mathrm{m}^{3}$ to $1.40 \times 10^{-4}$ $\mathrm{mol} / \mathrm{m}$. This range of bulk concentrations is chosen based on the dilution of the original ink concentration, where $1.40 \times 10^{-4} \mathrm{~mol} / \mathrm{m}^{4}$ represents the most concentrated ink available. 
Simulation information

\begin{tabular}{|l|l|}
\hline Name of the model & $\begin{array}{l}\text { Simulation of a reverse-emulsion electrophoretic device as } \\
\text { an electric double layers system }\end{array}$ \\
\hline Model type & 1-D steady state \\
\hline Multiphysics modules & Electrostatic coupled with transport of diluted species \\
\hline Variable & $\begin{array}{l}\text { Concentration } \\
\left(1.40 \cdot 10^{-4} \mathrm{~mol} / \mathrm{m}^{3} \text { as } 100 \% \text { concentrated to } 12.25 \%\right)\end{array}$ \\
\hline
\end{tabular}

\section{Simulation parameters}

\begin{tabular}{|l|l|}
\hline Voltage & $0.5 \mathrm{~V}$ \\
\hline Distance between electrodes & 10 microns \\
\hline Relative permittivity & 3 \\
\hline Temperature & $300 \mathrm{~K}$ \\
\hline Dynamic viscosity & $2.012 \mathrm{cP}[2.012 \mathrm{mPa} \cdot \mathrm{s}]$ \\
\hline Particle radius & $100 \mathrm{~nm}$ \\
\hline
\end{tabular}

Figure 20: Concentration effect on display contrast 1-D simulation informations and parameters.

\subsubsection{Nanodroplet concentration at the viewing plane}

The first simulation explores how bulk concentration of nanodroplets affects the spatial distribution of nanodroplets and counterions in the ink solution. The counterions are those that have opposite charge $(+)$ with respect to the ink nanodroplets (-) and are thereby repelled away from the viewing plane. The scenario simulates concentrations in the dark operating state, when nanodroplets are attracted to the electrode at the viewing plane.

For higher levels of bulk concentration, the steady-state concentration of nanodroplets outside of the electric double layers also increases, as shown in figure 21. The increase of nanodroplet ink concentration in the diffuse layer is undesirable when the display is switched to light state. Ideally, in an electrophoretic display, almost all of the (dark) dye-containing nanodroplets would be in the diffuse layer, where voltage has a strong influence. Likewise, for higher levels of bulk 

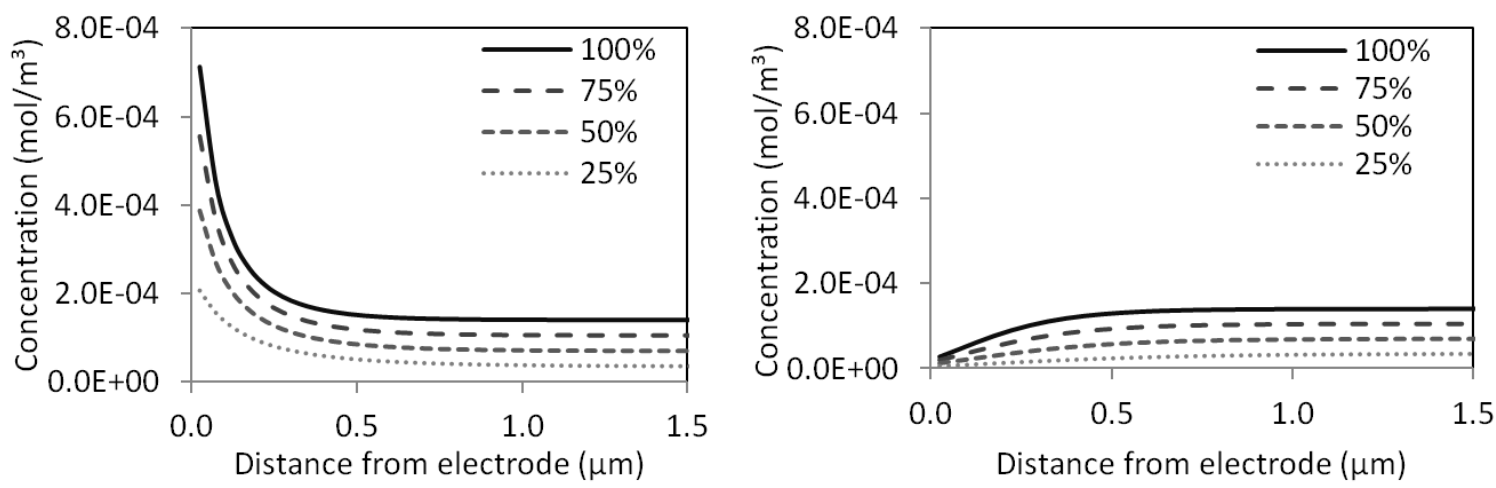

Figure 21: Concentration as a function of distance from electrode, for nanodroplets (left) and counterions (right), for different levels of bulk concentration.

concentration, the steady-state concentration of counterions outside of the electric double layers also increases. Ideally, in an electrophoretic display, it is desirable to have the counterions absent from regions closest to the viewing plane, so that more nanodroplets responsible for color can occupy that viewing region. In the dark state setting (positive charge at the electrode), the counterions close to the viewing plane indeed approach zero.

\subsubsection{Electric potential vs. distance from electrode}

Figure 22 shows the simulated electrical potential near the electrode at the viewing plane. Beyond approximately $1.5 \mu \mathrm{m}$, the potential reaches a very small value, close to zero. This simulation also shows that as the bulk concentration increases, the distance at which electrostatic screening occurs is reduced. Conversely, when the ionic solution is less concentrated, the electrostatic screening is less severe.

\subsubsection{Steric compensation}

"Steric compensation" refers to accounting for the space occupied by charged particles within an electric double layer system that otherwise would treat the ionic 


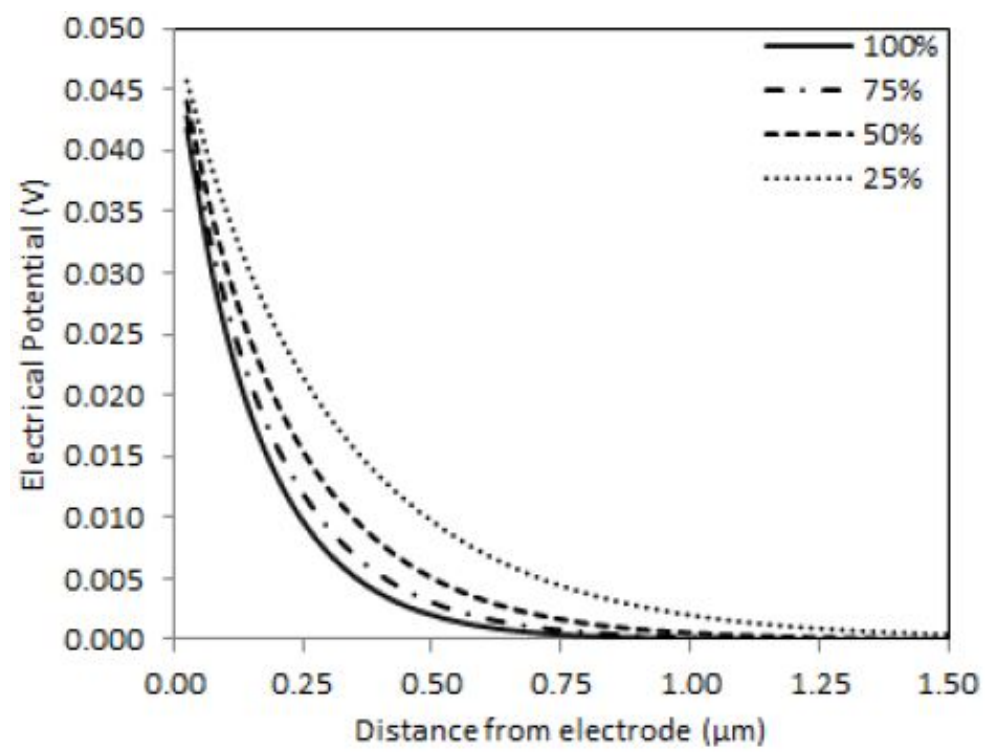

Figure 22: Electric potential versus distance from electrode, for a range of bulk concentration.

particles as merely point charges. When including the steric effect, however, assumptions such as packing structure, diffuse layer thickness, and effective transport volume are needed. An upper limit to nanodroplet concentration for the diffuse layer is calculated based on volume occupancy, assuming a hexagonal close packed (HCP) arrangement. Reverse micelles can occupy different packing densities in different phases. It is consistent with the findings of other published research that in a densely packed situation, the reverse micelles have an HCP arrangement $[139,140,141,142]$.

Knowing the diffuse layer thickness, device volume, packing efficiency, and available void space (accounting for $\mathrm{TiO}_{2}$ occupancy), the remaining space available inside the diffuse layer can be calculated. By dividing the space available inside the diffuse layer with nanodroplet volume, the maximum number of nanodroplets within the diffuse layer can be roughly estimated. A conservative estimate of $1 \mu \mathrm{m}$ is used for the thickness of the region close to an electrode, in which it is assumed that 
nanodroplets can achieve dense packing. Using a size estimate of roughly $200 \mathrm{~nm}$ (from dynamic light scattering), this represents a thickness approximately 5 monolayers deep.

Using these assumptions and rough estimates, figure 23 plots the calculated nanodroplet concentration in both dark and light states near the viewing plane. The upper curves represent the device operating in the dark state (i.e. the electrode having an opposite charge as that of nanodroplets), and the lower curves represent the light state.

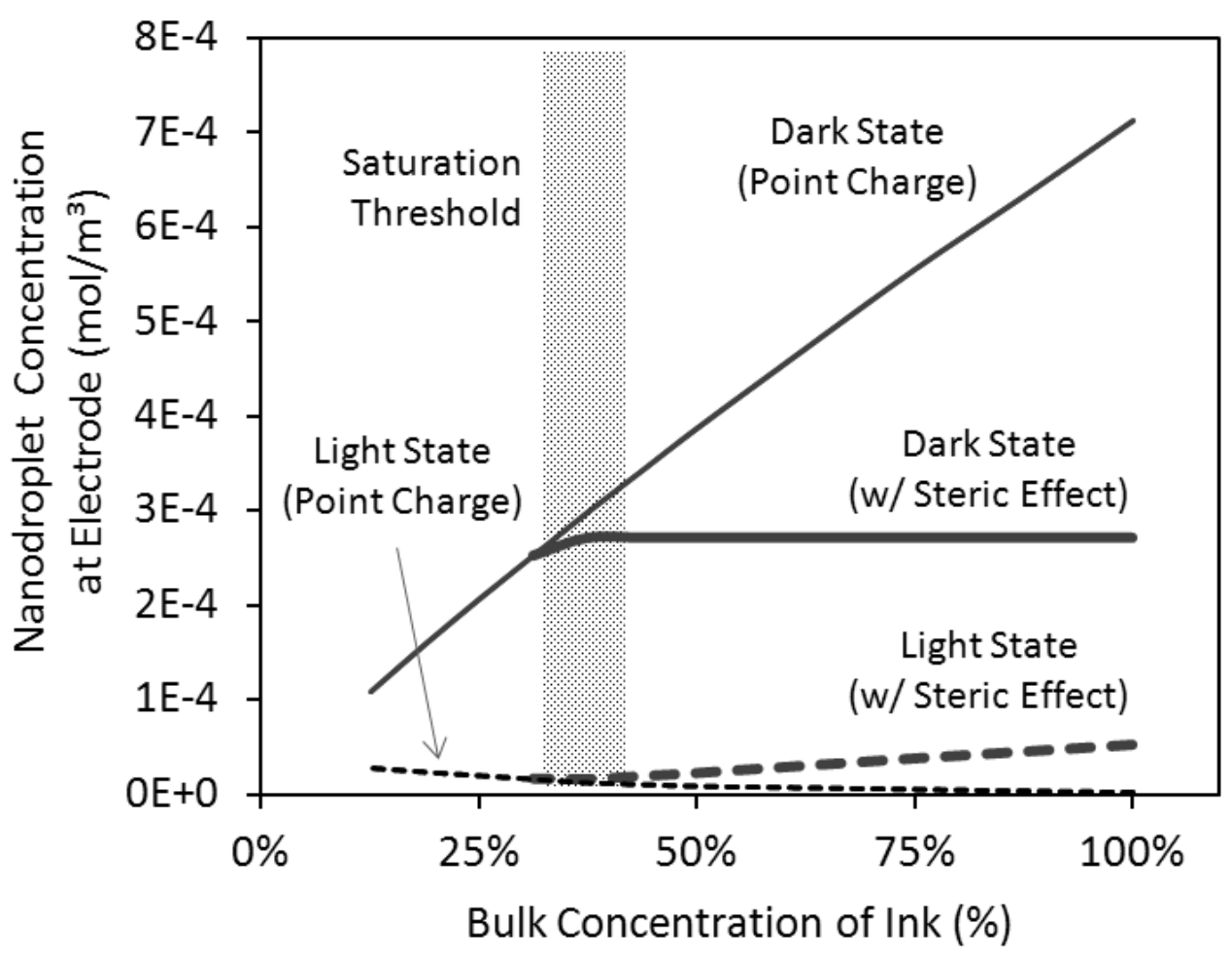

Figure 23: Poisson-Nernst-Planck (point charge) model of nanodroplet concentration near the viewing plane in dark state (solid line) and light state (dashed line).

Without steric effect and being in the dark state, the concentration of nanodroplets near the viewing plane increases proportionally to the concentration in 
the bulk, as expected. In the light state, the nanodroplet concentration decreases linearly with increasing bulk concentration and applied voltage. However, in reality, the concentration of nanodroplets near the electrode cannot increase indefinitely, and the steric effect must be considered.

From the lowest bulk concentration to a critical bulk concentration level at approximately $38 \%$, the nanodroplet concentration near the electrode increases. On the other hand, from the lowest bulk concentration to the same critical level, the nanodroplet concentration near the electrode decreases as the electric field drives the nanodroplet away from the viewing plane. Beyond this critical level, the Stern and diffuse layers are saturated. As figure 22 shows, the electric potential outside the diffuse layer approaches zero. Nanodroplets outside of the Stern and diffuse layers no longer feel the electric potential at the electrode. With steric effect considered, the nanodroplets' concentration near the viewing plane must be corrected because increasing the concentration in bulk past the critical concentration will only increase the free-flowing nanodroplet outside the Stern and diffuse layers. As shown in figure 23, the nanodroplet concentration increases after approximately $38 \%$ bulk concentration in the light state because of the ineffectiveness of the driving potential in the diffuse layer.

This simulation suggests that image contrast, depending on the ratio between the light and dark states, is highest at some intermediate concentration level. In other words, there is an optimal bulk concentration of nanodroplets in the ink that will permit the highest difference in concentration (and thus, highest difference in luminance) near the viewing plane. The existence of such optimum is proven experimentally, using the experimental test methods explained in the next chapter. 


\subsection{1-D vs. 2-D: Role of geometry in simulation}

In order to test the accuracy of the 1-D model, the nanodroplet concentration migration because of an electric field in a 2-D hexagonal porous model is simulated. The 1-D model overlooks the electric field distortion caused by geometry boundaries and the different dielectric properties between solids and fluids. Items within the dashed lines in figure 24 form a unit cell in the 2-D hexagonal porous lattice. This figure shows that volume fraction can be varied by changing the side length of the unit cell. As long as the side lengths stay the same, the channels created by these hexagonal structures will remain uniform. The model used in later simulations were of $40 \%$ porous volume fraction. Additionally, the 2-D model enables visual tortuosity of the porous lattice. Studies have shown that in the field of biomechanics, losses attributed to tortuosity, non-planarity and complex geometry can no longer be accounted for using a 1-D model as the system gets smaller and more complex [143].

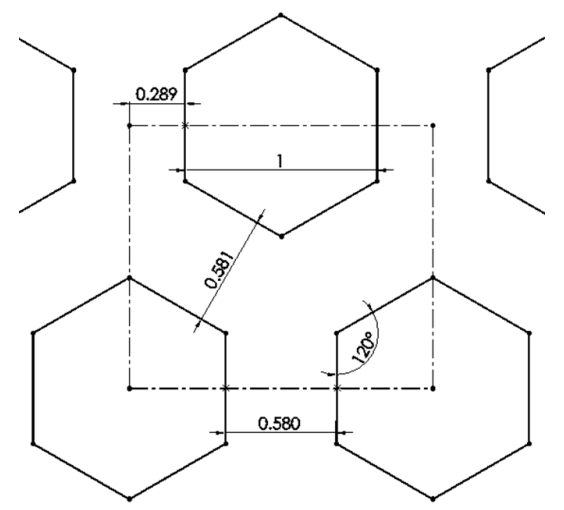

Figure 24: A unit cell as the base of the 2-D hexagonal porous lattice.

The simulation shows that electrostatic screening in the 1-D model presents little difference in value when compared with the 2-D model, even when the 


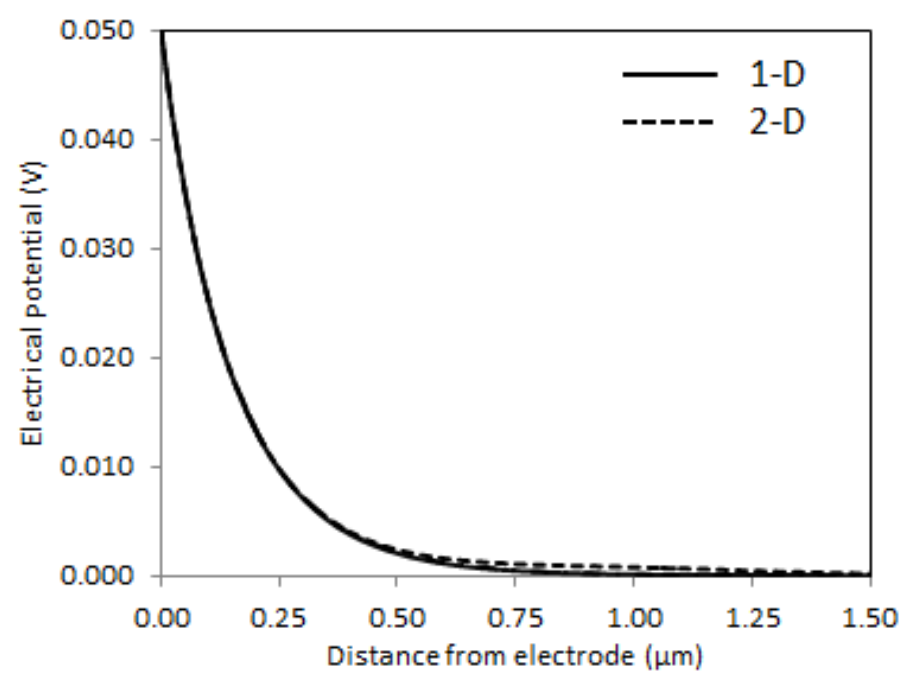

Figure 25: Electrostatic screening comparison at the electrode of 1-D and 2-D hexagonal porous models.

material and geometric properties are taken into account. This justifies the use of 1-D simulation in this particular system.

\subsubsection{3-D computed tomography}

Further simulation refinement can upgrade the 2-D model to a 3-D porous matrix structure with 3-D X-ray computed tomography (CT). With recent improvements of X-ray CT and robust modeling tools, it is possible to import 3-D specimen-specific geometry into finite element analysis or computational fluid dynamic software. Tomography is capable of imaging detailed 3-D solid objects by scanning the object section by section through penetrating waves [144, 145]. Studies done by John R. Izzo Jr. et al. incorporate the use of a reflective condenser optic lens to provide a spatial resolution in the tens of nanometers for a 3-D CT scan $[146,147]$. The 3-D structure can then be transferred into a multiphysics simulation program, to perform simulation. Similar methods have been used to study chemical 
transport in cracked concrete. The use of a 3-D image constructed by CT scans is a popular way to analyze heterogeneous microstructures [148]. The scope of this thesis does not include 3-D simulation, but subsequent studies for finer detail may take advantage of this capability.

\subsection{Transient analyses of display switching}

Transient analysis is needed to reveal the response of the display. To analyze the influence of geometry in device switching, both 1-D and 2-D hexagonal porous models are simulated. In the first sets of simulation, all the parameters are kept the same, but voltage is varied to test the response time of the display. The second set of simulations includes the transient aspect of the concentration effect on response time. Lastly, the parametric simulation of the display switching with particle tracking can reveal particle motion and other functional dependencies within the 2-D device.

\subsubsection{Effects of voltage on display switching}

In this study, simulation protocols are kept the same in most of the parameters, including concentration, material property, and boundary conditions. The only thing that is varied is the nanodroplet ink driving voltage potential. Voltage potential is varied from $0.01 \mathrm{~V}$ to 0.5 volt. The voltage effects on display switching are simulated with both the 1-D and 2-D models. The parameters for both tests are listed in figure 26, and the results are shown in figure 27 .

Figure 27 shows that at $10 \mathrm{mV}$, the concentration of the nanodroplet ink at the viewing plane eventually plateaus at $20 \mathrm{~mol} / \mathrm{m}^{3}$. And at $50 \mathrm{mV}$, the concentration at the viewing plane shoots up past the saturation threshold within 5 seconds. 
Simulation information

\begin{tabular}{|l|l|}
\hline Name of the model & $\begin{array}{l}\text { Simulation of a reverse-emulsion electrophoretic device } \\
\text { as the electric double layers system. }\end{array}$ \\
\hline Model type & 1-D and 2-D transient. Interval [0 to -200 seconds] \\
\hline Multiphysics modules used & Electrostatic coupled with transport of diluted species. \\
\hline Variable & $0.01 \mathrm{~V}, 0.05 \mathrm{~V}, 0.1 \mathrm{~V}, 0.5 \mathrm{~V}$ \\
\hline
\end{tabular}

Simulation parameters

\begin{tabular}{|l|l|}
\hline Concentration & $7.00 \cdot 10^{-5} \mathrm{~mol} / \mathrm{m}^{3}, 50 \%$ concentrated ink \\
\hline Distance between electrodes & $10 \mathrm{microns}$ \\
\hline Relative permittivity & 3 \\
\hline Temperature & $300 \mathrm{~K}$ \\
\hline Dynamic viscosity & $2.012 \mathrm{cP}[2.012 \mathrm{mPa} \cdot \mathrm{s}]$ \\
\hline Particle radius & $100 \mathrm{~nm}$ \\
\hline Diffusion coefficient & $1.2 \cdot 10^{-12} \mathrm{~m}^{2} / \mathrm{s}$ \\
\hline
\end{tabular}

Figure 26: List of information and parameters for the 1-D and 2-D voltage effects on display switching models.
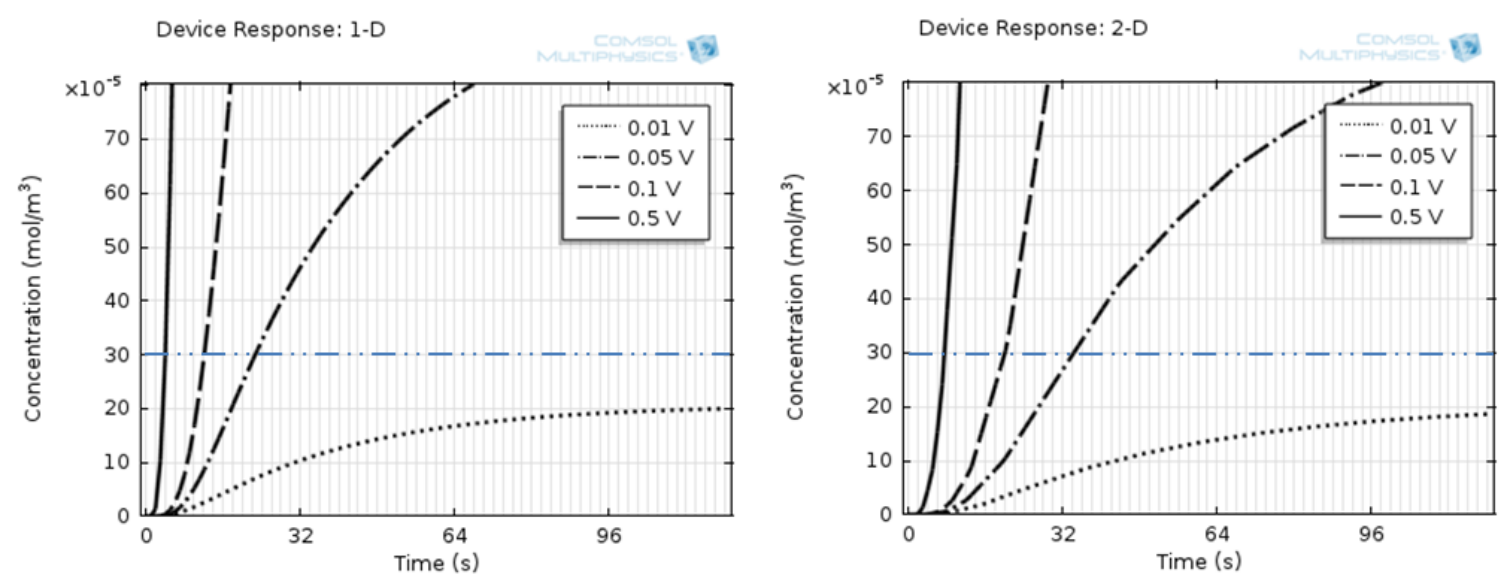

Figure 27: Ink concentration as a function of time and potential voltage at the electrode for 1-D (left) and 2-D (right) simulations.

5.4.2 Effect of nanodroplet ink concentration on display switching

In this study, voltage, material property, and boundary conditions are kept the same, while the nanodroplet ink concentration is varied between $12.5 \%$ and $100 \%$. 
The effect of concentration on device switching is inconclusive. Concentration is varied between $100 \%$ concentrated and $12.5 \%$. The concentration effects on display switching are simulated with both the 1-D and 2-D modes. Both sets of simulation parameters are listed in figure 28, and the results are shown in figure 29 and figure 30. Figures 29 and 30 also show that the ink nanodroplet concentration can vary the switching response in a reverse-emulsion electrophoretic display.

Simulation information

\begin{tabular}{|l|l|}
\hline Name of the model & $\begin{array}{l}\text { Simulation of a reverse-emulsion electrophoretic device } \\
\text { as the electric double layers system }\end{array}$ \\
\hline Model type & 1-D and 2-D transient, interval [0 to -200 seconds] \\
\hline Multiphysics modules & Electrostatic coupled with transport of diluted species \\
\hline Variable & $\begin{array}{l}\text { Concentration } \\
\left(1.40 \cdot 10^{-4} \mathrm{~mol} / \mathrm{m}^{3} \text { as } 100 \% \text { to } 12.25 \%\right)\end{array}$ \\
\hline
\end{tabular}

Simulation parameters

\begin{tabular}{|l|l|}
\hline Voltage & $0.5 \mathrm{~V}$ \\
\hline Distance between electrodes & 10 microns \\
\hline Relative permittivity & 3 \\
\hline Temperature & $300 \mathrm{~K}$ \\
\hline Dynamic viscosity & $2.012 \mathrm{cP}[2.012 \mathrm{mPa} \cdot \mathrm{s}]$ \\
\hline Particle radius & $100 \mathrm{~nm}$ \\
\hline Diffusion coefficient & $1.2 \cdot 10^{-12} \mathrm{~m}^{2} / \mathrm{s}$ \\
\hline
\end{tabular}

Figure 28: List of information and parameters for the 1-D and 2-D concentration effects on display switching models.

The simulation shows that the higher the concentration, the faster the switching response for the display. Figure 29 shows that at the same driving voltage, a device equipped with $100 \%$ concentrated ink reaches the saturation threshold within 10 seconds, whereas the $12.5 \%$ concentrated device took three times as long.

Figure 30 shows each device with various concentrations as the devices each reach their own steady state at different times. Devices with a lower concentration 

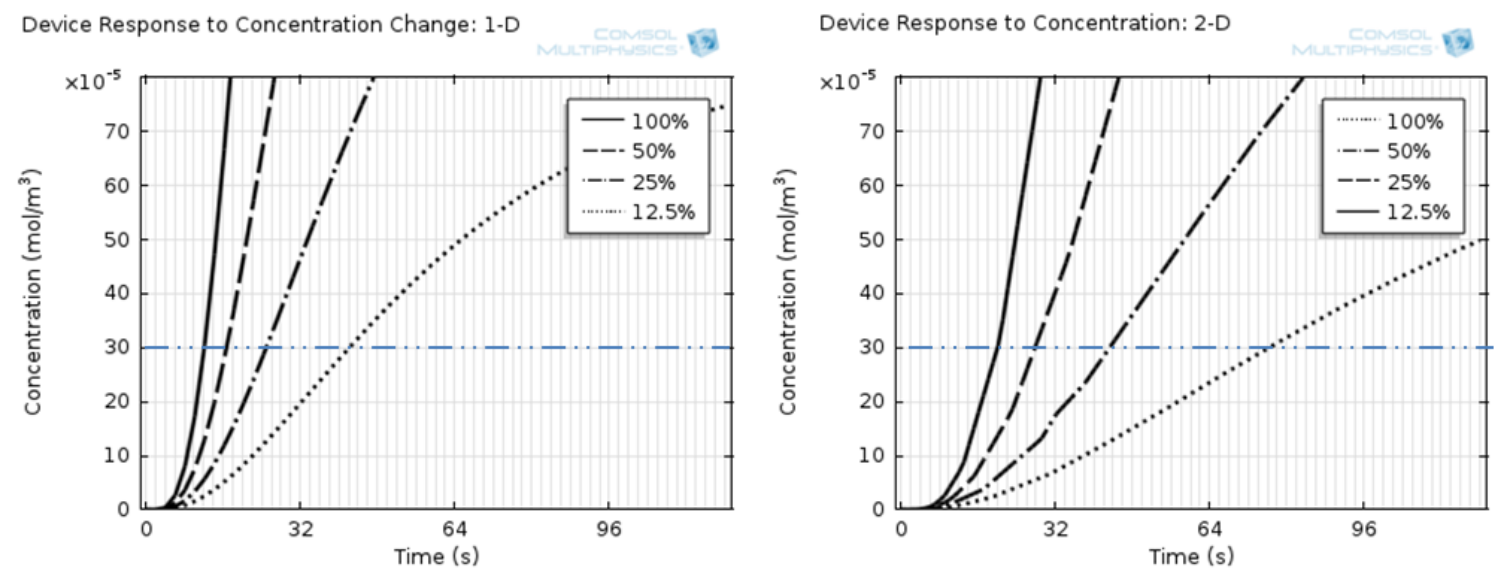

Figure 29: Ink nanodroplet concentration as a function of time and $\%$ bulk concentration at the electrode for the 1-D (left) and 2-D (right) simulations.

approach their steady state values slower than the devices with a higher concentration. Figure 30 also shows that each display has its own steady state concentration at the viewing plane. However, if this is not the actual case, the devices should have reached their maximum concentrations at $3.0 \times 10^{-} 4 \mathrm{~mole} / \mathrm{m}^{3}$ because of steric effect shown in section 5.2.3. The difference between voltage and concentration influence on device switching can also be shown from comparing figure 27 and figure 29. Voltage potential plays a bigger role than the concentration of the device on device switching. This can be seen by the switching speed variance from $0.01 \mathrm{~V}$ to $0.05 \mathrm{~V}$.

Differences between 1-D and 2-D models can also be seen from figure 27 and figure 29. A consistency of switching response lagging in 2-D models is apparent in both cases. This result verifies that the porous geometry within the device indeed provides obstructions to the mobile ink nanodroplets. 


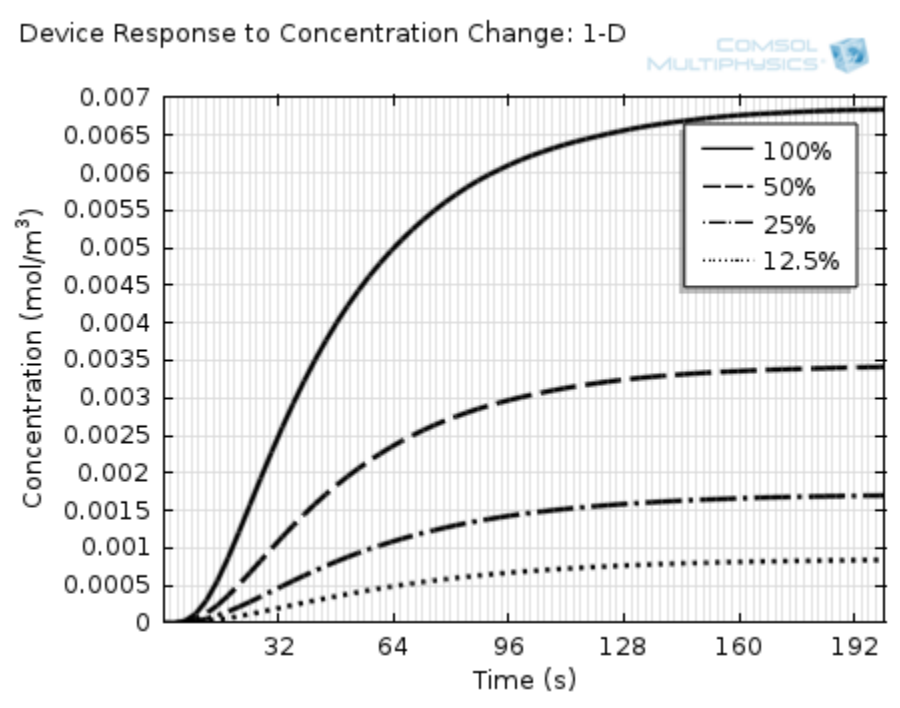

Figure 30: Ink nanodroplet concentrations at the electrode reaching its steady state for different $\%$ bulk concentrations with the same voltage potential.

\subsection{Parametric simulation of display switching}

Parametric performance modeling is conducted to provide insight into the functional dependencies between characteristic device parameters and response time. Geometry parameters such as the electrode gap and the solid fraction packing ratio of the porous matrix are inspected. For these simulations, response time is examined using a specialized particle-tracing module in the commercial multiphysics simulation software (COMSOL Multiphysics, COMSOL, Inc., Burlington, Massachusetts, USA). Normalized response time is quantified by the time required for a designated number of particles to travel through a 2-D hexagonal porous matrix from a source plane to a destination (i.e., viewing) plane divided by the thickness of the porous matrix. The simulated physics phenomena included electroosmotic flow in a porous medium and viscous drag. Particle-particle and particle-field interactions were not included in the simulations. The input factors were porous matrix thickness, solid volume fraction, and applied voltage. The solid 
volume fraction (dimensionless) is the proportion of space occupied by the $\mathrm{TiO}_{2}$ particles that comprise the porous matrix and is equal to unity minus porosity. The applied voltage, when divided by the layer thickness, can also be expressed as the electric field strength (in $\mathrm{V} / \mu \mathrm{m}$ ). Geometry constraint such as the size and morphology of the $\mathrm{TiO}_{2}$ particles were chosen to best match the scanning electron microscopy image. Initial attempts involved using an obstruction array of one-micron pillars within the simulated 2-D device. Solid volume fraction was determined by the spacing between each pillar, as particles could bounce off the structure as they came in contact with it when driven by the electric field created by the electric potential. Hexagons were used for the 2-D cross-sectional representation of the 3-D porous lattice. Each hexagon of the uniform porous matrix was designed to inscribe a 1 micron sphere by changing the channel gap. The solid volume fraction was determined using trigonometry. Hexagonal arrays were used instead as circular arrays because of a few distinct advantages. Circular arrays do not increase the similarity with scanning electron microscopy imaging. Many studies have also shown that in the case of nanocrystallization, it is common to have a densely packed hexagonal cell distribution $[149,150]$. And in finite element analysis, the mesh generated around circular objects tends to increase the computational power needed.

Figure 32 shows that the device-normalized response time is approximately linear with voltage. There is a diminishing effect on the normalized response time with the porous matrix thickness decreasing. And the device response time has no direct correlation with either $30 \%$ or $40 \%$ solid volume fraction. 


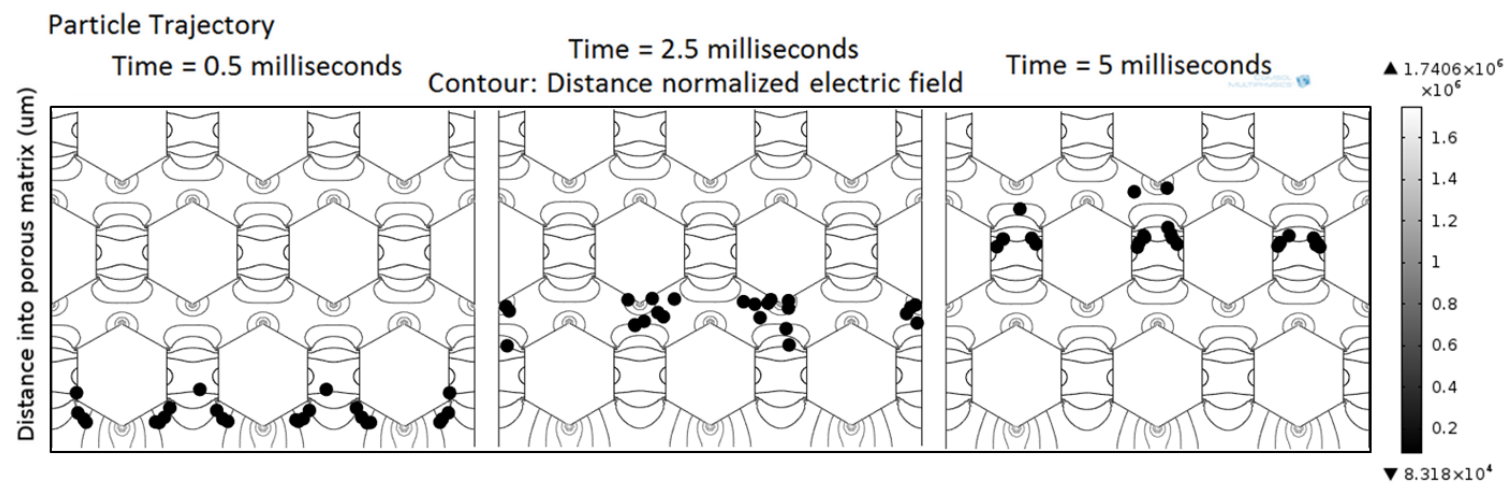

Figure 31: Particle trajectories simulation (30\% area fraction $\mathrm{TiO}_{2} 3 \mathrm{~V}$, particle charge number 1).
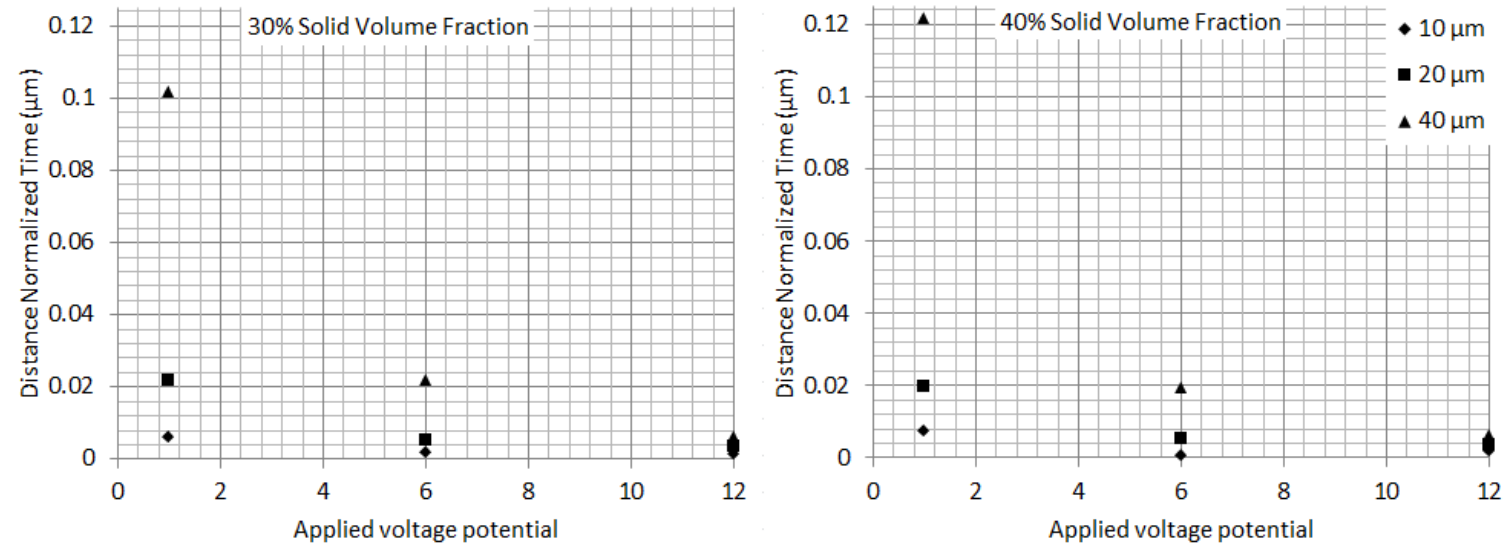

Figure 32: Comparison of distance normalized device response, for different combinations of input factors. 


\section{CHAPTER 6}

\section{EXPERIMENTAL TESTING}

The effects of ink concentration on image contrast and switching speed are determined by measuring the luminance of test devices loaded with different concentration levels. Luminance is defined as the luminous flux emitted from a surface per unit solid angle per unit area in a given direction and is therefore the luminous intensity per unit area. Luminance is measured in candela per square meter $(\mathrm{cd} / \mathrm{m})$ [151]. Relative luminance (used throughout this paper) is luminance in relation to a light standard and varies within a range from 0 (dark) to 1 (light). Different display manufacturers use different standards to describe the contrast of their device. However, the contrast of a device can generally be described by its contrast ratio or dynamic range. The International Committee for Display Metrology defines the peak contrast of a device as the ratio of the peak light luminance measurement, $L_{p}$, divided by the maximum full screen dark luminance, $L_{k}$, and is represented by the variable $C_{p}[152]$.

\subsection{Luminosity measurement}

The photodiode used is OPT101 from Texas Instruments. OPT101 is a monolithic photodiode with an on-chip transimpedance amplifier. The output voltage increases linearly with light intensity. The photodiode is prepared with basic circuit connections provided by the manufacturer, as shown in figure 33 . The apparatus used in this investigation measures light intensity with a photodiode interfaced to the data acquisition software LabVIEW (National Instruments, 


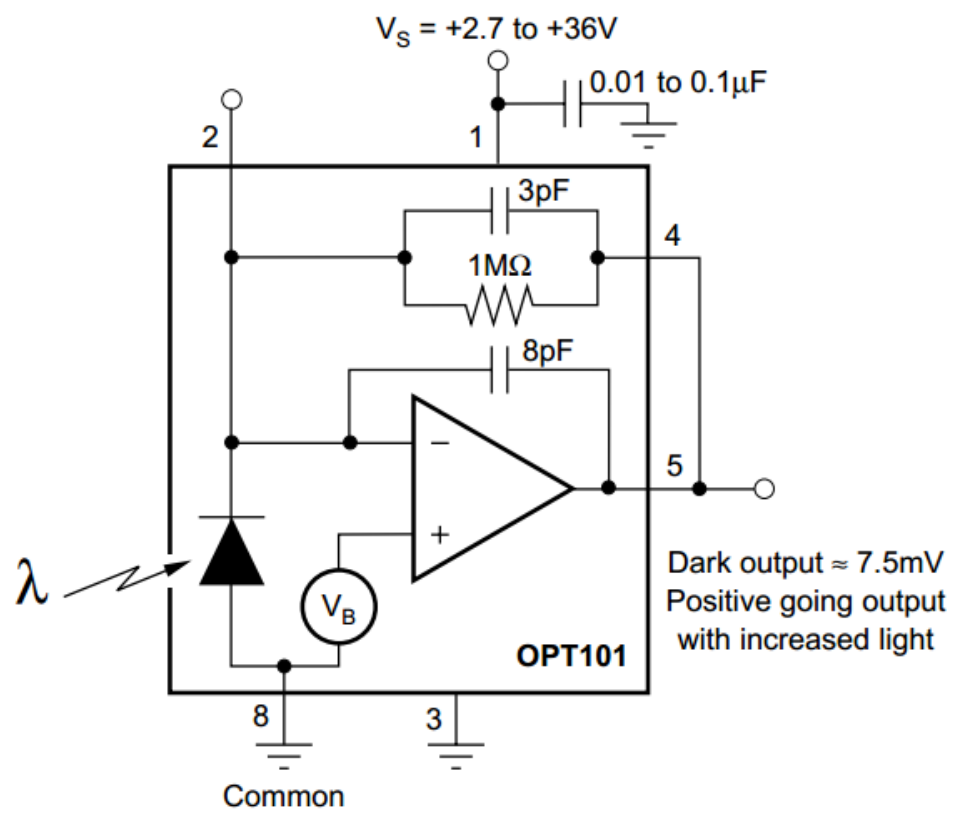

Figure 33: Circuit connections for the data acquisition device.

Austin, Texas, USA) and supporting hardware. Photodiode signals are calibrated to luminance using a grayscale calibration palette (X-Rite, Inc., Grand Rapids, Michigan, USA). As shown in figure 34, devices are held in a test fixture for consistent lighting and orientation as reflected intensity from the surface of the device is measured. Also shown in the figure is a $5 \mathrm{~mm}$ aperture to mask smaller regions of the test device, using opaque vinyl tape. The isolated regions are used to check spatial uniformity.

6.2 Ink concentration experiment

Test devices were fabricated by mixing the ink with $\mathrm{TiO}_{2}$ powder into a paste-like form. This paste was interposed between $40 \mathrm{~mm} \times 25 \mathrm{~mm}$ glass slides with ITO. A uniform gap was established by mixing a few $10 \mu \mathrm{m}$ glass spheres (Duke Standards 9000 Series, Thermo Fisher Scientific, Waltham, Massachusetts, 


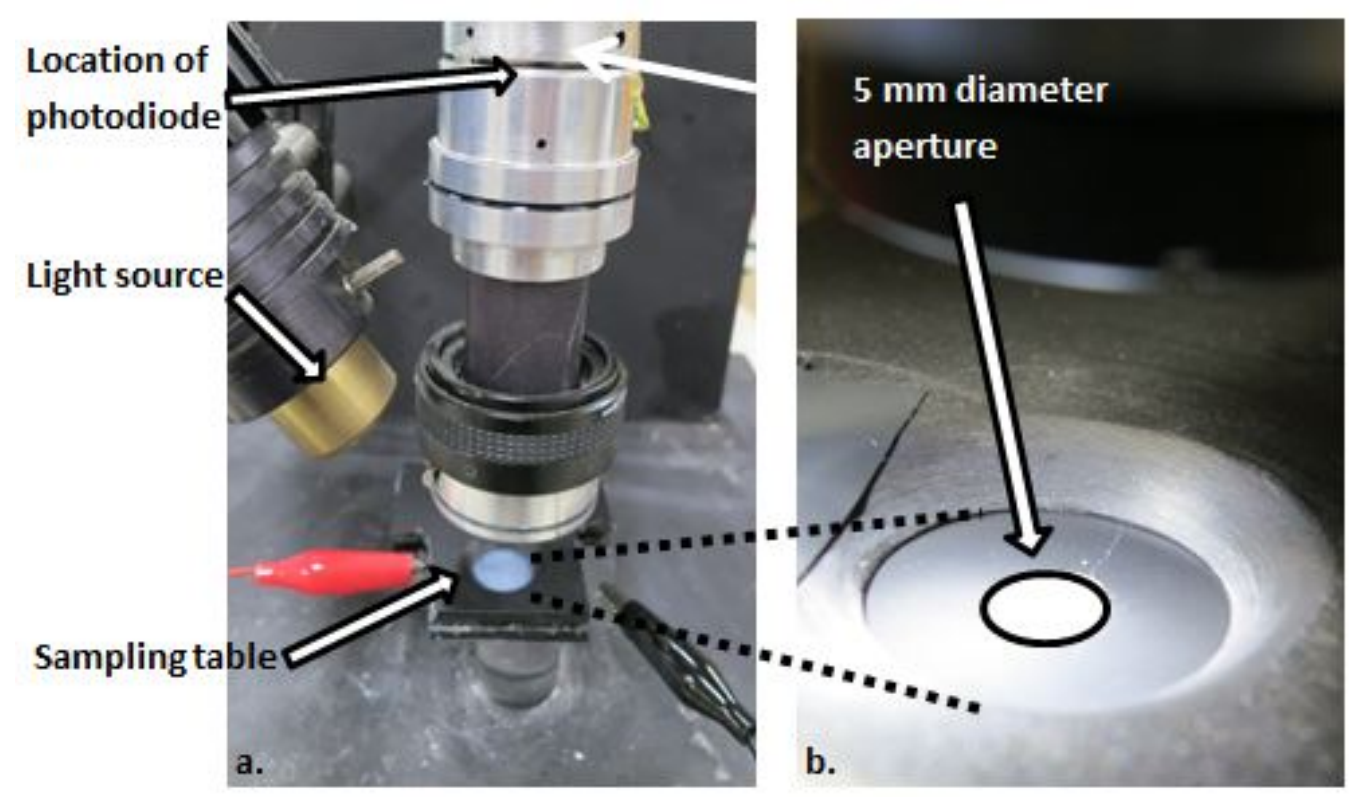

Figure 34: Test fixture with light source and photodiode (left), and close-up of the aperture (right).

USA) into the ink- $\mathrm{TiO}_{2}$ paste. The highest loading at full concentration (i.e., 100\%) is estimated to have $8.4 \times 10^{10}$ nanodroplets per $\mu \mathrm{L}$, which is equivalent to $1.4 \times 10^{-4} \mathrm{~mol} / \mathrm{m}$. The ink was prepared and tested at full concentration and 5 other dilutions in tetralin at concentrations of $50 \%, 37.5 \%, 25 \%, 18.75 \%$, and $12.5 \%$.

As a newly assembled device is operated repeatedly, relative luminance may not have the same exact value over sequential state changes. To deal with these varying values, each device is first conditioned by switching several times (typically 5 to 10 transitions) until the luminance between light and dark states becomes stable. The transient response, indicative of switching speed, is measured by plotting luminance as a function of time. The devices are switched by reversing polarities over a $2.5 \mathrm{~V}$ potential difference between electrodes. The polarity is reversed every 10 seconds, which is equivalent to a square-wave frequency of $0.05 \mathrm{~Hz}$. 


\subsection{Voltage experiment}

The concentration experiment is able to reveal the contrast behavior at different levels of concentration. However, even if the experimental results are similar to the predicted hypothesis, it would not verify that the observations are caused by concentration alone. To further reinforce the hypothesis, a voltage experiment is conducted. The hypothesis states that the contrast of the device is dictated by concentration and steric effects. If the hypothesis is correct, a higher-than-needed voltage should not strongly influence the contrast of the display. If the voltage experiment results show that maximum contrast improves as voltage increases indefinitely, then voltage has a significant role compared to concentration; steric effects, in this case, is inadequate for accurately representing behavior of the system.

Three devices are loaded with the same concentration. The devices are cycled between the light and dark states at 20-second intervals to ensure that even at low voltage the devices have the chance to reach their respective peak lightness and darkness. Each device is tested at three different locations for spatial variability. The voltage is then varied from $1 \mathrm{~V}, 3 \mathrm{~V}, 5 \mathrm{~V}$, and lastly, $8 \mathrm{~V}$. At each voltage level, a minimum of three cycles between the light and dark states are recorded with a photodetector. 


\section{CHAPTER 7}

\section{RESULTS AND DISCUSSION}

\subsection{Image contrast}

To examine the effect of ink concentration on image contrast, figure 35 plots relative luminance as a function of ink concentration for both the light and dark states. The plotted data includes measured values collected over 50 seconds of a square-wave alternating signal, and the relative luminance values from all three locations on a given device are averaged. The dense plotting of values at each higher extreme of luminance corresponds to the device when held in its light state, and the dense plotting of values at the lower extreme corresponds to its dark state. The intermediate data points at each concentration are from the transitions between the light and dark states. As indicated in the figure, the greatest difference between the light and dark states is observed at an intermediate concentration (25\%), revealing that there is indeed an optimum concentration for image contrast.

Contrast is expressed here in terms of peak contrast, defined by the International Committee for Display Metrology as the ratio of peak light luminance

divided by maximum full screen dark luminance [151]. Figure 36 plots contrast as a function of concentration, reinforcing the observation that the best contrast is achieved using $25 \%$ concentration.

\subsection{Switching speed}

Transient response of the devices is measured to examine the effect of ink concentration on switching speed. Figure 37 plots relative luminance versus time for 


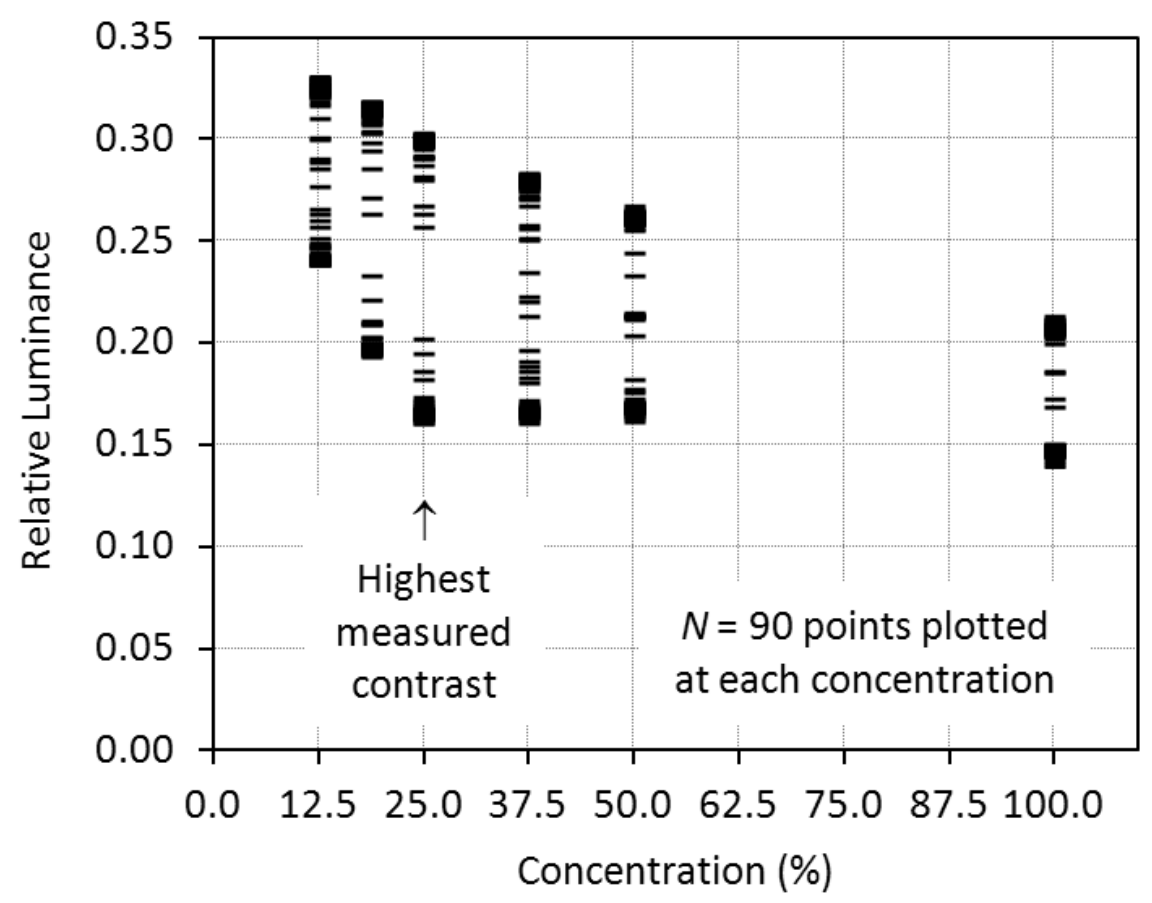

Figure 35: Relative luminance versus ink concentration.

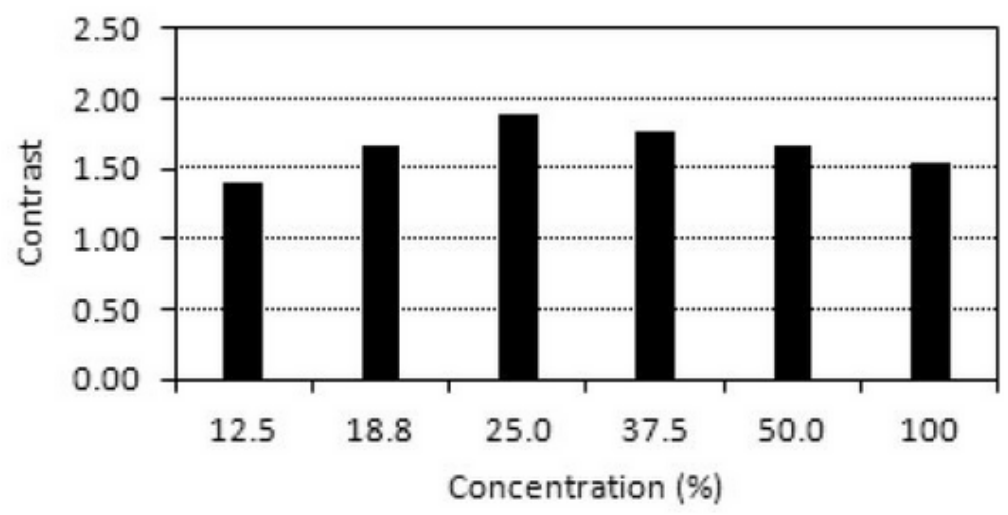

Figure 36: Contrast versus ink concentration.

two light-to-dark switching transitions, for each of the different ink concentrations. The applied potential difference is $2.5 \mathrm{~V}$ in all cases, and the luminance values from all three locations on a given device are averaged. Differences in switching speed based on concentration are relatively small compared with the more significant 
differences observed in image contrast. As shown here, the intermediate case of $25 \%$ ink concentration has the best contrast, and its transient characteristics are similar to the time response of the other concentrations.

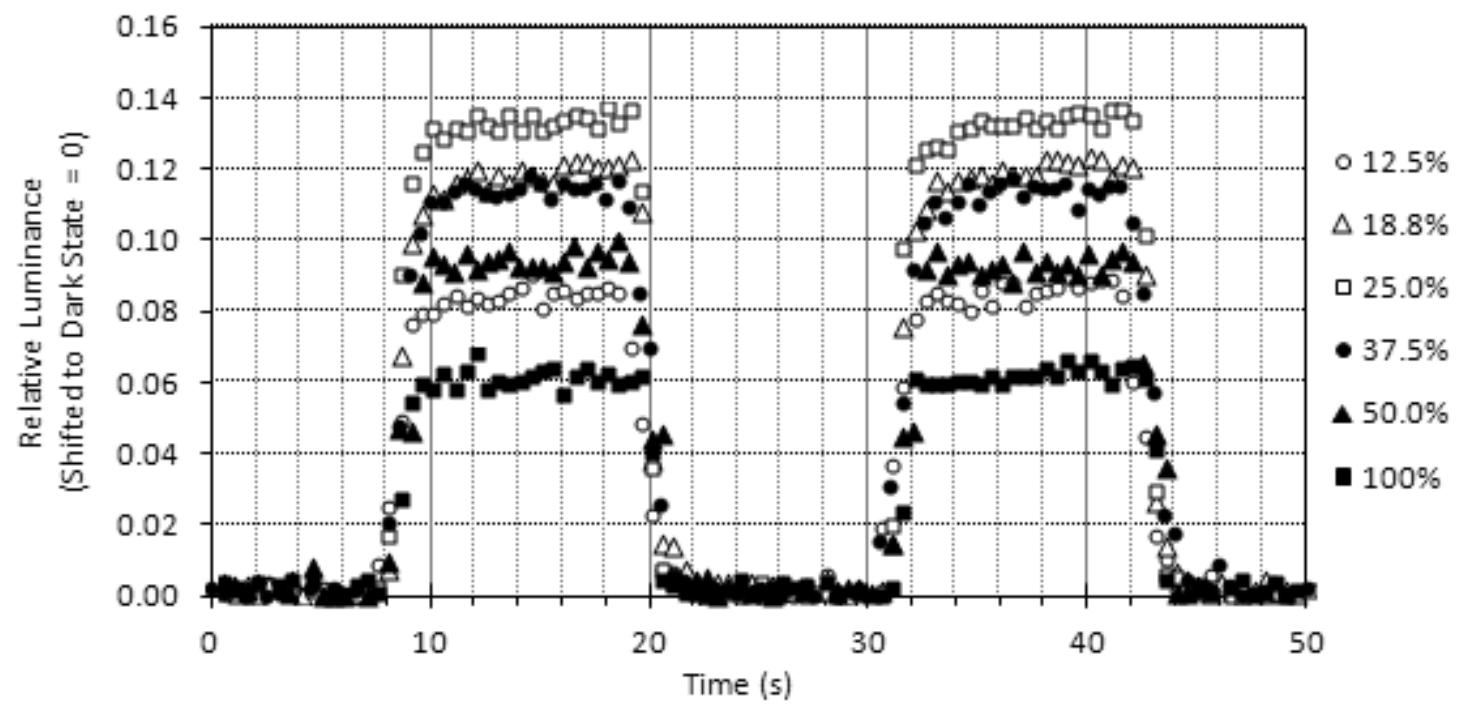

Figure 37: Relative luminance versus time for two light-to-dark switching transitions, using different ink concentrations.

Although some of the plotted data has a slight upward trend even after 10 seconds, all of the different concentration levels reveal very similar steady-state plateaus beginning within the first 2 seconds. This suggests that with respect to ink concentration, switching speed is substantially less dependent on concentration, relative to how strongly image contrast depends on concentration. From a device design perspective, this means that the ink concentration may be selected to achieve the best contrast, with little or negligible adverse effect on switching speed.

\subsection{Spatial variability}

Each device was tested at three different locations using a circular 5-millimeter-diameter mask to isolate test regions. Figure 38 shows the spatial 
variability for the devices at each of the concentration levels. The vertical axes on all six plots have the same relative luminance range of 0.2 but have been offset from zero luminance to focus on differences based on imaging location. The $12.5 \%$ and $50.0 \%$ concentration levels exhibited the largest spatial variability, while the $18.8 \%$ and $25 \%$ concentration levels showed good spatial uniformity.

\subsection{Comparison with simulation}

In the multiphysics simulation, the bulk concentration of droplets within the device was varied over the same range that was used experimentally in order to observe the effect on device contrast. Steric effect is added to the model of electric double layers. Peak contrast achieved at the $25 \%$ concentration level is reasonably close to the $38 \%$ saturation value suggested by the simulation. Gray lines in figure 39 provide an alternative orientation of the simulation prediction in figure 23. This shows the concentration effect on device luminance, with darkness increasing as the concentration level at the viewing plane increases. In figure 39, the result predicted by the simulation overlaps with the experimental result in figure 35. Both show similar concentration saturation behavior as darkness cannot be increased once the concentration saturation threshold is reached. The light state (upper portion of figure 35) darkens as the nanodroplet bulk concentration increases.

\subsection{Voltage experiments}

Single-pixel devices were loaded with ink that had the same concentration. These devices were cycled at voltages leveling from $1 \mathrm{~V}$ to $8 \mathrm{~V}(1 \mathrm{~V}, 3 \mathrm{~V}, 5 \mathrm{~V}, 8 \mathrm{~V})$. This experiment was performed to examine the voltage effects on device contrast and response time. Similar to the data recorded in the concentration study in 


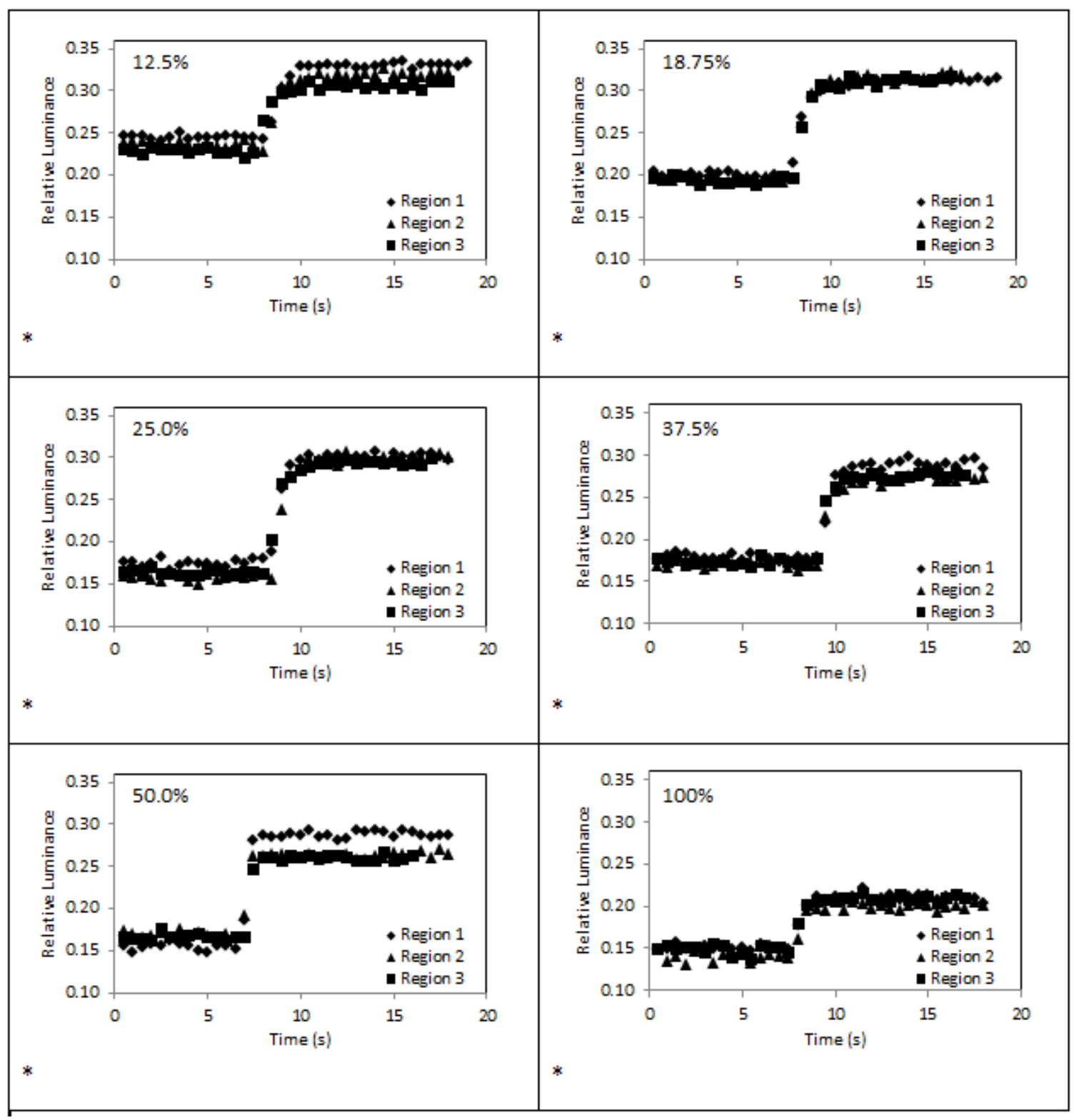

Figure 38: Variability in relative luminance at three distinct locations per device, for each concentration level.

section 7.2 , the gathered luminance data showed the device cycling between the light to the dark state. To extract contrast at different voltage values, the data were organized with a similar technique in the dense plotting performed in figure 35.

Figure 40 shows the dense plotting of a single-pixel device switching between 


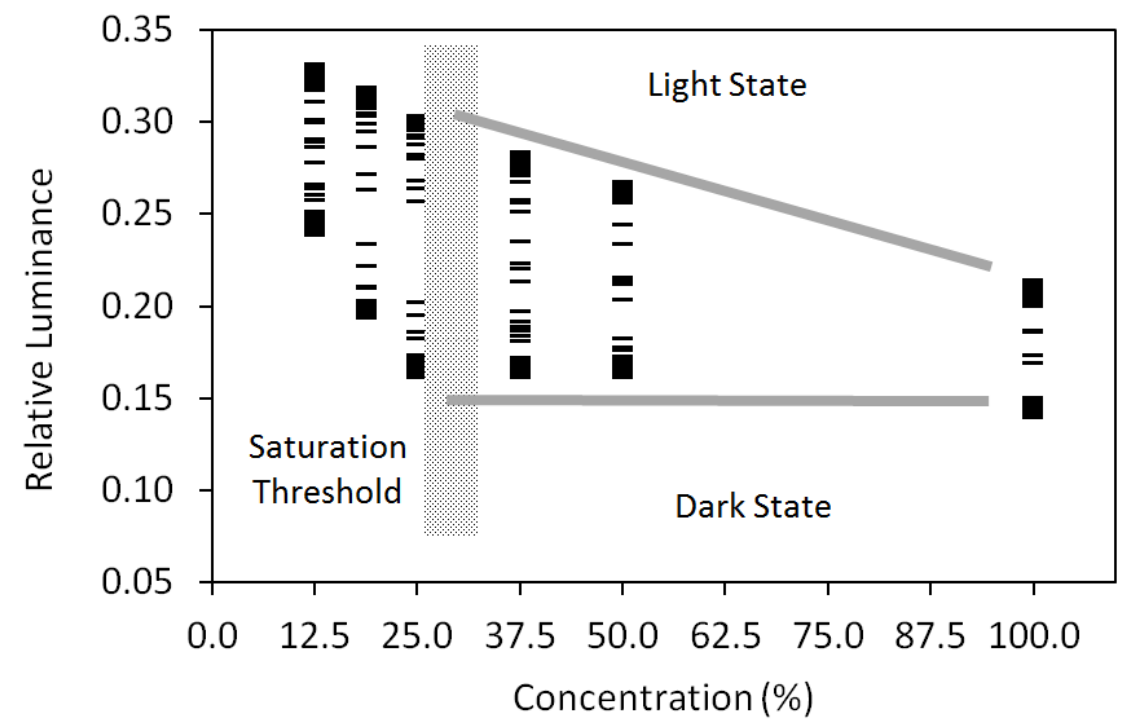

Figure 39: Comparison of the simulation prediction and experimental result. Plot of experimental relative luminance versus concentration, with characteristics similar to calculated nanodroplet concentration near the viewing plane (as in figure 23).

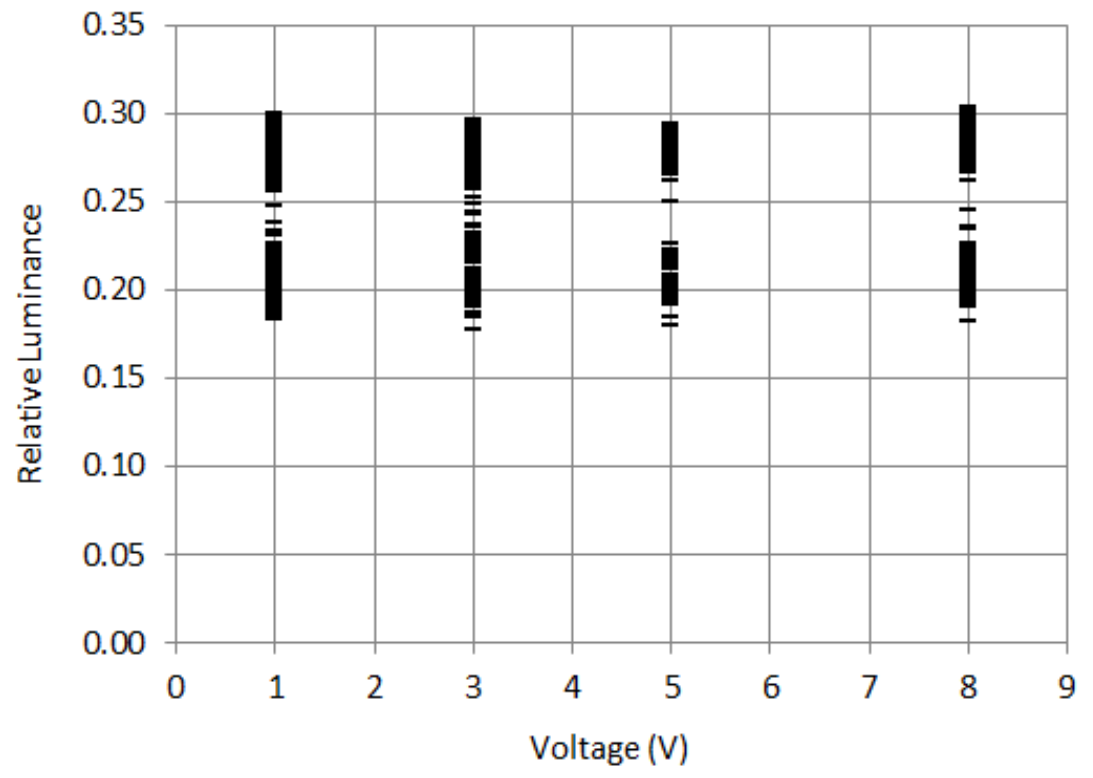

Figure 40: Relative luminance versus applied voltage with devices at the same concentration. 
the light state to the dark state at different voltages. The devices show consistent contrast throughout all four voltages tested regardless of the increase in driving voltage. The result further concluded that even at $1 \mathrm{~V}$, the voltage applied to the device was sufficient for driving a reverse-emulsion electrophoretic display. 


\section{CHAPTER 8}

\section{CONCLUSIONS AND FUTURE WORK}

The findings of this study support the hypothesis that bulk concentration of nanodroplets in the ink is a crucial parameter for optimizing device contrast in a reverse-emulsion electrophoretic display. Simulations of nanodroplet concentration at the viewing plane agreed with experimentally measured values of luminance, specifically in terms of saturation threshold. Simulation and experimental evidence both show that merely increasing the bulk concentration of nanodroplets in the ink will not categorically achieve higher contrast, because contrast is limited by steric effects and electrostatic screening. Nanodroplets reach a saturation level in the Stern and diffuse layers. Increasing bulk concentration excessively only increases free-flowing nanodroplets outside of the diffuse layer, providing no further benefits in the dark state. These free-flowing nanodroplets also diminish the ability to achieve maximum luminance in the light state.

A higher voltage would be needed to overcome electrostatic screening. However, a higher voltage may cause adverse effects, such as, dissociation of the nanodroplets. Furthermore, increasing voltage only compensates for electrostatic screening and does not bypass the steric effect. This explains why the results showed no significant improvement of contrast even at a higher voltage, further supporting the hypothesis that concentration and steric effects dictate maximum contrast.

In the characterization portion of the study, dynamic light scattering was used to determine nanodroplet size, resulting in an average radius of approximately 176 nm. This value was almost twice as much as the dry samples prepared for atomic 
force microscopy. The relative permittivity of the ink was determined to be 3.0, measured by RC circuit experiment. As expected, this value is close to the relative permittivity of tetralin, which is the solvent used for the ink.

There is additional investigation that can be done to further support the hypothesis. This includes possibly working with a modified Poisson-Nernst-Planck equation to simulate the steric effect with a finer resolution and establishing a quantitative correlation between nanodroplet concentration and relative luminosity. Measurements with finer time resolutions can be done with transient analyses for a better quantitative understanding of the concentration effects on switching response. 


\section{REFERENCES}

[1] J. Heikenfeld, P. Drzaic, J.-S. Yeo, and T. Koch, "Review paper: A critical review of the present and future prospects for electronic paper," Journal of the Society for Information Display, vol. 19, no. 2, pp. 129-156, 2011.

[2] J. D. Albert and B. Comiskey, "Shutter mode microencapsulated electrophoretic display," Jan. 9 2001. US Patent 6,172,798.

[3] R. Zehner, K. Amundson, A. Knaian, B. Zion, M. Johnson, and G. Zhou, "20.2: Drive waveforms for active matrix electrophoretic displays," SID Symposium Digest of Technical Papers, vol. 34, no. 1, pp. 842-845, 2003.

[4] G. Feng, J. Barrus, K. F. Gudan, and S. Chemishkian, "46.2: Real-time pen tracking on electronic paper displays," SID Symposium Digest of Technical Papers, vol. 39, no. 1, pp. 689-692, 2008.

[5] G. Feng and M. J. Gormish, "46.4: Ghosting reduction using digital halftoning for electrophoretic displays," SID Symposium Digest of Technical Papers, vol. 39, no. 1, pp. 697-700, 2008.

[6] R. J. Paolini Jr, S. J. Telfer, A. Bouchard, and J. Atkinson, "Tetrachromatic color filter array for reflective display," Oct. 11 2012. US Patent $20,120,257,269$.

[7] V. Akwukwuma and F. Chete, "Electronic-paper: The electronic display of the future," The Pacific Journal of Science and Technology, vol. 13, no. 2, pp. 173-180, 2012.

[8] G. Duthaler, J. Au, M. Davis, H. Gates, B. Hone, A. Knaian, E. Pratt, K. Suzuki, S. Yoshida, M. Ueda, et al., "53.1: Active-matrix color displays using electrophoretic ink and color filters," in SID Symposium Digest of Technical Papers, vol. 33, pp. 1374-1377, Wiley Online Library, 2002.

[9] M. Bryning and R. Cromer, "37.4: Reverse-emulsion electrophoretic display (reed)," in SID Symposium Digest of Technical Papers, vol. 29, pp. 1018-1021, Wiley Online Library, 1998.

[10] C. Yu, B. Tian, J. Fan, G. D. Stucky, and D. Zhao, "Synthesis of siliceous hollow spheres with ultra large mesopore wall structures by reverse emulsion templating.," Chemistry Letters, no. 1, pp. 62-63, 2002. 
[11] H. G. Yang and H. C. Zeng, "Creation of intestine-like interior space for metal-oxide nanostructures with a quasi-reverse emulsion," Angewandte Chemie International Edition, vol. 43, no. 39, pp. 5206-5209, 2004.

[12] M. Bryning, Z. Bryning, and R. Cromer, "Novel systems, methods and compositions relating to display elements," Apr. 18 2008. US Patent App. $12 / 105,927$.

[13] M. Ahumada, M. Bryning, R. Cromer, M. Hartono, and S. Lee, "A thin porous substrate using bonded particles for reverse-emulsion electrophoretic displays," in SPIE OPTO, pp. 82800R-82800R, International Society for Optics and Photonics, 2012.

[14] J. L. Mateusz Bryning, Remy Cromer, "Low-cost, flexible electronic paper," Apr. 182011.

[15] M. Bryning and R. Cromer, "Reverse emulsion electrophoretic display - a revolutionary approach for flexible electronic paper.," in SID 26th International Display Research Conference, pp. 402-405, 2006.

[16] O. P. Gupta and R. C. Ricketson, "Shelf price label and product placement verification method and apparatus," Feb. 20 1996. US Patent 5,493,107.

[17] H. T. Keh and S. Y. Park, "To market, to market: the changing face of grocery retailing," Long Range Planning, vol. 30, no. 6, pp. 836-846, 1997.

[18] D. Graham-Rowe, "Electronic paper rewrites the rulebook for displays," Nature Photonics, vol. 1, no. 5, pp. 248-251, 2007.

[19] F. marketing institute, "Supermarket facts," cited 2013 May 142013. Avaliable.

[20] Pricer, "Benefits," cited 2013 May 12 2013. Avaliable.

[21] N. Seppa, "Urban eyes: Too much time spent indoors may be behind a surge in nearsightedness," Science News, vol. 183, no. 3, pp. 22-25, 2013.

[22] B. Comiskey, J. Albert, H. Yoshizawa, and J. Jacobson, "An electrophoretic ink for all-printed reflective electronic displays," Nature, vol. 394, no. 6690, pp. 253-255, 1998.

[23] J. Jacobson, B. Comiskey, and J. Albert, "Microencapsulated electrophoretic display," Oct. 5 1999. US Patent 5,961,804.

[24] H. Gates, R. Zehner, H. Doshi, and J. Au, "31.2: A5 sized electronic paper display for document viewing," SID Symposium Digest of Technical Papers, vol. 36, no. 1, pp. 1214-1217, 2005. 
[25] J. Gurski and L. M. Quach, "Display technology overview," Lytica Streamlining Progress, 2005.

[26] E. Ink, "Technology," cited 2013 May 12 2002. Avaliable.

[27] Y. Chen, K. Denis, P. Kazlas, and P. Drzaic, "12.2: A conformable electronic ink display using a foil-based a-si tft array," SID Symposium Digest of Technical Papers, vol. 32, no. 1, pp. 157-159, 2001.

[28] I. Shen, K.-K. Shieh, C.-Y. Chao, D.-S. Lee, et al., "Lighting, font style, and polarity on visual performance and visual fatigue with electronic paper displays," Displays, vol. 30, no. 2, pp. 53-58, 2009.

[29] Y.-T. Lin, P.-H. Lin, S.-L. Hwang, S.-C. Jeng, and C.-C. Liao, "Investigation of legibility and visual fatigue for simulated flexible electronic paper under various surface treatments and ambient illumination conditions," Applied Ergonomics, vol. 40, no. 5, pp. 922-928, 2009.

[30] H. Isono, S. Takahashi, Y. Takiguchi, and C. Yamada, "Measurement of visual fatigue from reading on electronic paper," The Journal of the Institute of Image Information and Television Engineers, vol. 59, no. 3, pp. 403-406, 2005.

[31] P. Kazlas, J. Au, K. Geramita, H. Gates, M. Steiner, C. Honeyman, P. Drzaic, K. Schleupen, B. Wisnieff, R. Horton, and R. John, "12.1: 12.1 svga microencapsulated electrophoretic active matrix display for information appliances," SID Symposium Digest of Technical Papers, vol. 32, no. 1, pp. 152-155, 2001.

[32] A. J. K. P. R. A. G. H. M. M. Chen, Y., "Electronic paper: Flexible active-matrix electronic ink display," Nature, vol. 423, no. 6936, p. 136, 2003.

[33] P. Drzaic and R. J. Wilcox, "Full color reflective display with multichromatic sub-pixels," mar 2005 2005. US Patent 6,864,875.

[34] J. D. Albert and H. G. Gates, "Microencapsulated electrophoretic display with integrated driver," Nov. 22 2005. US Patent 6,967,640.

[35] R. A. Hayes and B. Feenstra, "Video-speed electronic paper based on electrowetting," Nature, vol. 425, no. 6956, pp. 383-385, 2003.

[36] R. Sakurai, S. Ohno, S.-i. Kita, Y. Masuda, and R. Hattori, "68.2: Color and flexible electronic paper display using qr-lpd technology," SID Symposium Digest of Technical Papers, vol. 37, no. 1, pp. 1922-1925, 2006.

[37] H. You and A. Steckl, "Three-color electrowetting display device for electronic paper," Applied Physics Letters, vol. 97, no. 2, pp. 023514-023514-3, 2010. 
[38] R. Hattori, S. Yamada, Y. Masuda, and N. Nihei, "20.3: Novel type of bistable reflective display using quick response liquid powder," in SID Symposium Digest of Technical Papers, vol. 34, pp. 846-849, Wiley Online Library, 2003.

[39] R. Hattori, S. Yamada, Y. Masuda, and N. Nihei, "A novel bistable reflective display using quick-response liquid powder," Journal of the Society for Information Display, vol. 12, no. 1, pp. 75-80, 2004.

[40] R. Sakurai, R. Hattori, M. Asakawa, T. Nakashima, I. Tanuma, A. Yokoo, N. Nihei, and Y. Masuda, "A flexible electronic-paper display with an ultra-thin and flexible lsi driver using quick-response liquid-powder technology," Journal of the Society for Information Display, vol. 16, no. 1, pp. 155-160, 2008.

[41] R. J. Mortimer, "Organic electrochromic materials," Electrochimica Acta, vol. 44, no. 18, pp. 2971-2981, 1999.

[42] D. Corr, U. Bach, D. Fay, M. Kinsella, C. McAtamney, F. O’Reilly, S. Rao, and N. Stobie, "Coloured electrochromic paper-quality displays based on modified mesoporous electrodes," Solid State Ionics, vol. 165, no. 1, pp. 315-321, 2003.

[43] E. Unur, P. M. Beaujuge, S. Ellinger, J.-H. Jung, and J. R. Reynolds, "Black to transmissive switching in a pseudo three-electrode electrochromic device," Chemistry of Materials, vol. 21, no. 21, pp. 5145-5153, 2009.

[44] D. Cummins, G. Boschloo, M. Ryan, D. Corr, S. N. Rao, and D. Fitzmaurice, "Ultrafast electrochromic windows based on redox-chromophore modified nanostructured semiconducting and conducting films," The Journal of Physical Chemistry B, vol. 104, no. 48, pp. 11449-11459, 2000.

[45] M. Grätzel, "Materials science: Ultrafast colour displays," Nature, vol. 409, no. 6820, pp. 575-576, 2001.

[46] A. Henzen, N. Ailenei, F. Van Reeth, G. Vansichem, R. W. Zehner, and K. Amundson, "32.4: An electronic ink low latency drawing tablet," SID Symposium Digest of Technical Papers, vol. 35, no. 1, pp. 1070-1073, 2004.

[47] K. Amundson, J. Ewing, P. Kazlas, R. McCarthy, J. Albert, R. Zehner, P. Drzaic, J. Rogers, Z. Bao, and K. Baldwin, "12.3: Flexible, active-matrix display constructed using a microencapsulated electrophoretic material and an organic-semiconductor-based backplane," in SID Symposium Digest of Technical Papers, vol. 32, pp. 160-163, Wiley Online Library, 2001.

[48] H. Zang and R. Liang, "Microcup electronic paper by roll-to-roll manufacturing processes," The Spectrum, vol. 16, no. 2, pp. 16-21, 2003. 
[49] K. Shinozaki, "5.5 l: Late-news paper: Electrodeposition device for paper-like displays," in SID Symposium Digest of Technical Papers, vol. 33, pp. 39-41, Wiley Online Library, 2002.

[50] T. Koch, D. Hill, M. Delos-Reyes, J. Mabeck, J.-S. Yeo, J. Stellbrink, D. Henze, and Z.-L. Zhou, "49.5 l: Late-news paper: Roll-to-roll manufacturing of electronic skins," in SID Symposium Digest of Technical Papers, vol. 40, pp. 738-741, Wiley Online Library, 2009.

[51] J.-S. Yeo, T. Emery, G. Combs, V. Korthuis, J. Mabeck, R. Hoffman, T. Koch, Z.-L. Zhou, and D. Henze, "69.4: Novel flexible reflective color media integrated with transparent oxide tft backplane," in SID Symposium Digest of Technical Papers, vol. 41, pp. 1041-1044, Wiley Online Library, 2010.

[52] T. Koch, J.-S. Yeo, Z.-L. Zhou, Q. Liu, J. Mabeck, G. Combs, V. Korthuis, R. Hoffman, B. Benson, and D. Henze, "Novel flexible reflective color media with electronic inks," Journal of Information Display, vol. 12, no. 1, pp. 5-10, 2011 .

[53] B. B. Q. Liu, T. R. Koch, J. Mabeck, R. L. Hoffman, D. A. Mourey, G. Combs, Z.-L. Zhou, and D. Henze, "52.4 l: Late-news paper: Ultra-low-power reflective display with world's best color," in SID Symposium Digest of Technical Papers, vol. 43, pp. 708-710, Wiley Online Library, 2012.

[54] T. Bert and H. De Smet, "The microscopic physics of electronic paper revealed," Displays, vol. 24, no. 3, pp. 103-110, 2003.

[55] D. P. Barz, M. J. Vogel, and P. H. Steen, "Determination of the zeta potential of porous substrates by droplet deflection. i. the influence of ionic strength and ph value of an aqueous electrolyte in contact with a borosilicate surface," Langmuir, vol. 25, no. 3, pp. 1842-1850, 2009.

[56] T. Bert, F. Beunis, H. D. Smet, and K. Neyts, "Steady state current in epids," Displays, vol. 27, no. 1, pp. 35-38, 2006.

[57] K. Neyts, M. Karvar, O. Drobchak, T. Brans, F. Strubbe, and F. Beunis, "Simulation of charge transport and steady state in non-polar media between planar electrodes with insulating layers," Colloids and Surfaces A:

Physicochemical and Engineering Aspects, no. 0, pp. -, 2012.

[58] K. Neyts, F. Beunis, F. Strubbe, M. Marescaux, B. Verboven, M. Karvar, and A. Verschueren, "Charge transport and current in non-polar liquids," Journal of Physics: Condensed Matter, vol. 22, no. 49, p. 494108, 2010.

[59] B. Conway, Electrochemical supercapacitors: scientific fundamentals and technological applications (POD). Kluwer Academic/plenum. New York, 1999. 
[60] S. L. Carnie, D. Y. Chan, and J. S. Gunning, "Electrical double layer interaction between dissimilar spherical colloidal particles and between a sphere and a plate: The linearized poisson-boltzmann theory," Langmuir, vol. 10, no. 9, pp. 2993-3009, 1994.

[61] J.-P. Hansen and H. Lowen, "Effective interactions between electric double-layers," arXiv preprint cond-mat/0002295, 2000.

[62] H. Nakanishi, D. A. Walker, K. J. Bishop, P. J. Wesson, Y. Yan, S. Soh, S. Swaminathan, and B. A. Grzybowski, "Dynamic internal gradients control and direct electric currents within nanostructured materials," Nature Nanotechnology, vol. 6, no. 11, pp. 740-746, 2011.

[63] M. S. Kilic, M. Z. Bazant, and A. Ajdari, "Steric effects in the dynamics of electrolytes at large applied voltages. i. double-layer charging," Physical Review E, vol. 75, no. 2, p. 021502, 2007.

[64] B. Li and D. Y. Kwok, "Electrokinetic microfluidic phenomena by a lattice boltzmann model using a modified poisson-boltzmann equation with an excluded volume effect," The Journal of chemical physics, vol. 120, p. 947, 2004.

[65] M. E. Parent, J. Yang, Y. Jeon, M. F. Toney, Z.-L. Zhou, and D. Henze, "Influence of surfactant structure on reverse micelle size and charge for nonpolar electrophoretic inks," Langmuir, vol. 27, no. 19, pp. 11845-11851, 2011.

[66] M. Kaszuba, J. Corbett, F. M. Watson, and A. Jones, "High-concentration zeta potential measurements using light-scattering techniques," Philosophical Transactions of the Royal Society A: Mathematical, Physical and Engineering Sciences, vol. 368, no. 1927, pp. 4439-4451, 2010.

[67] B. A. Todd and J. A. Cohen, "Separability of electrostatic and hydrodynamic forces in particle electrophoresis," Physical Review E, vol. 84, no. 3, p. 032401, 2011.

[68] W. B. Russel, D. A. Saville, and W. R. Schowalter, Colloidal dispersions. Cambridge University Press, 1992.

[69] B. J. Yoon and S. Kim, "Electrophoresis of spheroidal particles," Journal of colloid and interface science, vol. 128, no. 1, pp. 275-288, 1989.

[70] S. Durand-Vidal, J.-P. Simonin, and P. Turq, Electrolytes at interfaces, vol. 1. Kluwer Academic Print on Demand, 2001. 
[71] M. Fixman, "Thin double layer approximation for electrophoresis and dielectric response@ f@f," The Journal of Chemical Physics, vol. 78, p. 1483, 1983.

[72] W. Chew, "Dielectric enhancement and electrophoresis due to an electrochemical double layer: a uniform approximation," The Journal of chemical physics, vol. 80, p. 4541, 1984.

[73] C. Z. IV and D. Saville, "The interpretation of electrokinetic measurements using a dynamic model of the stern layer: I. the dynamic model," Journal of Colloid and Interface Science, vol. 114, no. 1, pp. 32 - 44, 1986.

[74] R. O'Brien and D. N. Ward, "The electrophoresis of a spheroid with a thin double layer," Journal of colloid and interface science, vol. 121, no. 2, pp. 402-413, 1988.

[75] H. Ohshima and K. Furusawa, Electrical Phenomena and Interfaces: Fundamentals, Measurements, and Applications. Surfactant Science, Marcel Dekker Incorporated, 1998.

[76] B. B. Damaskin and O. A. Petrii, "Historical development of theories of the electrochemical double layer," Journal of Solid State Electrochemistry, vol. 15, no. 7-8, pp. 1317-1334, 2011.

[77] "Nernst equations," Phys. Chem Z, vol. 2, p. 613, 1888.

[78] O. W, Electrochemistry history. Obrazovanie, St. Petersburg, 1911.

[79] "Helmholtz theory," Pogg Ann Phys, vol. 89, p. 228, 1853.

[80] "Helmholtz dl theory," Wied AnnPhys, vol. 7, p. 337, 1879.

[81] "Gouy-champman dl thoery," Physique, vol. 9, p. 475, 1910.

[82] "Gouy-champman edl," Physique, vol. 9, p. 475, 1910.

[83] "Stern layer edl," Z Elektrochem, vol. 30, p. 508, 1924.

[84] H. Wennerström, B. Jönsson, and P. Linse, "The cell model for polyelectrolyte systems. exact statistical mechanical relations, monte carlo simulations, and the poisson-boltzmann approximation," The Journal of Chemical Physics, vol. 76, p. 4665, 1982.

[85] M. E. Davis and J. A. McCammon, "Solving the finite difference linearized poisson-boltzmann equation: A comparison of relaxation and conjugate gradient methods," Journal of Computational Chemistry, vol. 10, no. 3, pp. 386-391, 1989. 
[86] W. Rocchia, E. Alexov, and B. Honig, "Extending the applicability of the nonlinear poisson-boltzmann equation: Multiple dielectric constants and multivalent ions," The Journal of Physical Chemistry B, vol. 105, no. 28, pp. 6507-6514, 2001.

[87] G. Gouy, "Constitution of the electric charge at the surface of an electrolyte," J. phys, vol. 9, no. 4, pp. 457-467, 1910.

[88] D. L. Chapman, "Li. a contribution to the theory of electrocapillarity," The London, Edinburgh, and Dublin Philosophical Magazine and Journal of Science, vol. 25, no. 148, pp. 475-481, 1913.

[89] I. DieMethodezurBestimmungderosmotischenZustandsgleichung, "Zur theorie der elektrolyte.," 1924.

[90] B. Derjaguin and L. Landau, "Theory of the stability of strongly charged lyophobic sols and the adhesion of strongly charged particles in solutions of electrolytes.," Acta Physicochim, vol. 14, pp. 633-662, 1941.

[91] E. J. W. Verwey and J. T. G. Overbeek, Theory of the stability of lyophobic colloids. Courier Dover Publications, 1999.

[92] M. E. Davis and J. A. McCammon, "Electrostatics in biomolecular structure and dynamics," Chemical Reviews, vol. 90, no. 3, pp. 509-521, 1990.

[93] B. Honig and A. Nicholls, "Classical electrostatics in biology and chemistry," Science, vol. 268, no. 5214, pp. 1144-1149, 1995.

[94] A. MacGillivray, "Nernst-planck equations and the electroneutrality and donnan equilibrium assumptions," The journal of chemical physics, vol. 48, p. 2903, 1968.

[95] T. R. Brumleve and R. P. Buck, "Numerical solution of the nernst-planck and poisson equation system with applications to membrane electrochemistry and solid state physics," Journal of Electroanalytical Chemistry and Interfacial Electrochemistry, vol. 90, no. 1, pp. 1-31, 1978.

[96] D. Gillespie, W. Nonner, and R. S. Eisenberg, "Coupling poisson-nernst-planck and density functional theory to calculate ion flux," Journal of Physics: Condensed Matter, vol. 14, no. 46, p. 12129, 2002.

[97] R. F. Probstein, Physicochemical Hydrodynamics. John Wiley \& Sons, 2005.

[98] H. Park, J. Lee, and T. Kim, "Comparison of the nernst-planck model and the poisson-boltzmann model for electroosmotic flows in microchannels," Journal of colloid and interface science, vol. 315, no. 2, pp. 731-739, 2007. 
[99] C. W. Outhwaite, L. B. Bhuiyan, and S. Levine, "Theory of the electric double layer using a modified poisson-boltzman equation," J. Chem. Soc., Faraday Trans. 2, vol. 76, pp. 1388-1408, 1980.

[100] C. W. Outhwaite and L. B. Bhuiyan, "An improved modified poisson-boltzmann equation in electric-double-layer theory," Journal of the Chemical Society, Faraday Transactions 2: Molecular and Chemical Physics, vol. 79 , no. 5, pp. 707-718, 1983.

[101] C. Outhwaite and L. Bhuiyan, "A modified poisson-boltzmann analysis of the electric double layer around an isolated spherical macroion," Molecular Physics, vol. 74, no. 2, pp. 367-381, 1991.

[102] M. G. Kurnikova, R. D. Coalson, P. Graf, and A. Nitzan, "A lattice relaxation algorithm for three-dimensional poisson-nernst-planck theory with application to ion transport through the gramicidin a channel," Biophysical Journal, vol. 76 , no. 2, pp. 642-656, 1999.

[103] Z. Schuss, B. Nadler, and R. S. Eisenberg, "Derivation of poisson and nernst-planck equations in a bath and channel from a molecular model," Physical Review E, vol. 64, no. 3, p. 036116, 2001.

[104] D. Chen, J. Jerome, R. Eisenberg, and V. Barcilon, "Qualitative properties of steady-state poisson-nernst-planck systems: Perturbation and simulation study," SIAM Journal on Applied Mathematics, vol. 57, no. 3, pp. 631-648, 1997.

[105] J. Park and J. Jerome, "Qualitative properties of steady-state poisson-nernst-planck systems: Mathematical study," SIAM Journal on Applied Mathematics, vol. 57, no. 3, pp. 609-630, 1997.

[106] H. Daiguji, P. Yang, and A. Majumdar, "Ion transport in nanofluidic channels," Nano Letters, vol. 4, no. 1, pp. 137-142, 2004.

[107] D. Stein, M. Kruithof, and C. Dekker, "Surface-charge-governed ion transport in nanofluidic channels," Physical Review Letters, vol. 93, no. 3, p. 035901, 2004 .

[108] J. J. McKetta, Encyclopedia of chemical processing and design, vol. 36. CRC Press, 1991.

[109] I. Borukhov, D. Andelman, and H. Orland, "Steric effects in electrolytes: A modified poisson-boltzmann equation," Physical review letters, vol. 79, no. 3, pp. 435-438, 1997. 
[110] I. Borukhov, D. Andelman, and H. Orland, "Adsorption of large ions from an electrolyte solution: a modified poisson-boltzmann equation," Electrochimica Acta, vol. 46, no. 2, pp. 221-229, 2000.

[111] G. Seth Roberts, T. A. Wood, W. J. Frith, and P. Bartlett, "Direct measurement of the effective charge in nonpolar suspensions by optical tracking of single particles," The Journal of chemical physics, vol. 126, no. 19, pp. 194503-194503, 2007.

[112] O. Otto, C. Gutsche, F. Kremer, and U. F. Keyser, "Optical tweezers with 2.5 khz bandwidth video detection for single-colloid electrophoresis," Review of Scientific Instruments, vol. 79, p. 023710, 2008.

[113] F. Strubbe, F. Beunis, and K. Neyts, "Detection of elementary charges on colloidal particles," Physical review letters, vol. 100, no. 21, p. 218301, 2008.

[114] R. Kemp, R. Sanchez, K. J. Mutch, and P. Bartlett, "Nanoparticle charge control in nonpolar liquids: Insights from small-angle neutron scattering and microelectrophoresis," Langmuir, vol. 26, no. 10, pp. 6967-6976, 2010.

[115] F. Beunis, F. Strubbe, K. Neyts, and D. Petrov, "Beyond millikan: the dynamics of charging events on individual colloidal particles," Physical review letters, vol. 108, no. 1, p. 016101, 2012.

[116] H. Sawada, Y. Tanishiro, N. Ohashi, T. Tomita, F. Hosokawa, T. Kaneyama, Y. Kondo, and K. Takayanagi, "Stem imaging of 47-pm-separated atomic columns by a spherical aberration-corrected electron microscope with a $300-\mathrm{kv}$ cold field emission gun," Journal of electron microscopy, vol. 58, no. 6, pp. 357-361, 2009.

[117] K. Takayanagi, Y. Oshima, T. Tanaka, Y. Tanihshiro, H. Sawada, F. Hosokawa, T. Tomita, T. Kaneyama, and Y. Kondo, "Lithium atom microscopy at sub-50pm resolution by r005," 2010.

[118] L. Zhang and A. Eisenberg, "Aggregates of polystyrene-b-poly (acrylic acid) block copolymers," Science, vol. 268, p. 23, 1995.

[119] N. S. Cameron, M. K. Corbierre, and A. Eisenberg, "1998 ewr steacie award lecture asymmetric amphiphilic block copolymers in solution: a morphological wonderland," Canadian journal of chemistry, vol. 77, no. 8, pp. 1311-1326, 1999.

[120] D. M. Willard and N. E. Levinger, "Influence of morphology on polar solvation dynamics in lecithin reverse micelles," The Journal of Physical Chemistry B, vol. 104, no. 47, pp. 11075-11080, 2000. 
[121] Y.-Y. Won, A. K. Brannan, H. T. Davis, and F. S. Bates, "Cryogenic transmission electron microscopy (cryo-tem) of micelles and vesicles formed in water by poly (ethylene oxide)-based block copolymers," The Journal of Physical Chemistry B, vol. 106, no. 13, pp. 3354-3364, 2002.

[122] V. Arcoleo and V. T. Liveri, "Afm investigation of gold nanoparticles synthesized in water/aot/n-heptane microemulsions," Chemical physics letters, vol. 258, no. 1, pp. 223-227, 1996.

[123] J. C. Yu, H. Y. Tang, J. Yu, H. C. Chan, L. Zhang, Y. Xie, H. Wang, and S. Wong, "Bactericidal and photocatalytic activities of tioj subi $2 \mathrm{i} / \mathrm{sub}_{\mathrm{i}}$, thin films prepared by sol-gel and reverse micelle methods," Journal of Photochemistry and Photobiology A: Chemistry, vol. 153, no. 1, pp. 211-219, 2002.

[124] R. Singh, J. Doolittle, M. A. George, and P. K. Dutta, "Novel surface structure of microporous faujasitic-like zincophosphate crystals grown via reverse micelles," Langmuir, vol. 18, no. 21, pp. 8193-8197, 2002.

[125] M. Regenbrecht, S. Akari, S. Förster, and H. Möhwald, "Shape investigations of charged block copolymer micelles on chemically different surfaces by atomic force microscopy," The Journal of Physical Chemistry B, vol. 103, no. 32, pp. 6669-6675, 1999.

[126] M. J. Fulwyler, "Fulwyler particle separator," Apr. 30 1968. US Patent $3,380,584$.

[127] A. Waggoner, "Fluorescent probes for analysis of cell structure, function, and health by flow and imaging cytometry," Applications of Fluorescence in the Biomedical Sciences, pp. 3-28, 1986.

[128] W. Sun, F. Ye, M. E. Gallina, J. Yu, C. Wu, and D. T. Chiu, "Lyophilization of semiconducting polymer dot bioconjugates," Analytical chemistry, 2013.

[129] S. Andreasen, S.-F. Chong, B. M. Wohl, K. N. Goldie, and A. N. Zelikin, "Poly (vinyl alcohol) physical hydrogel nanoparticles, not polymer solutions, exert inhibition of nitric oxide synthesis in cultured macrophages," Biomacromolecules, 2013.

[130] J. P. Blitz, J. L. Fulton, and R. D. Smith, "Dynamic light scattering measurements of reverse micelle phases in liquid and supercritical ethane," The Journal of Physical Chemistry, vol. 92, no. 10, pp. 2707-2710, 1988.

[131] J. L. Fulton, J. P. Blitz, J. M. Tingey, and R. D. Smith, "Reverse micelle and microemulsion phases in supercritical xenon and ethane: light scattering and 
spectroscopic probe studies," The Journal of Physical Chemistry, vol. 93, no. 10 , pp. 4198-4204, 1989.

[132] H. Bohidar and M. Behboudnia, "Characterization of reverse micelles by dynamic light scattering," Colloids and Surfaces A: Physicochemical and Engineering Aspects, vol. 178, no. 1, pp. 313-323, 2001.

[133] K. Kundu and B. K. Paul, "Physicochemical investigation of biocompatible mixed surfactant reverse micelles: Ii. dynamics of conductance percolation, energetics of droplet clustering, effect of additives and dynamic light scattering studies," The Journal of Chemical Thermodynamics, 2013.

[134] Malvern, "Zetasizer nano zs," cited 2013 May 30 2013. Avaliable.

[135] D. Viswanath, T. Ghosh, D. L. Prasad, N. Dutt, and K. Rani, "Experimental data," 2007.

[136] T. M. Aminabhavi and B. Gopalakrishna, "Densities, viscosities, and refractive indices of bis (2-methoxyethyl) ether+ cyclohexane or $+1,2,3$, 4-tetrahydronaphthalene and of 2-ethoxyethanol+ propan-1-ol,+ propan-2-ol, or+ butan-1-ol," Journal of Chemical and Engineering Data, vol. 40, no. 2, pp. 462-467, 1995.

[137] M. Paredes, R. Reis, A. Silva, R. Santos, G. Santos, M. Ribeiro, and P. Ximango, "Densities, sound velocities, and refractive indexes of (tetralin $+\mathrm{i}$ $\mathrm{i}_{i}, \mathrm{n}_{\mathrm{i}} / \mathrm{i}_{\mathrm{i}}$-decane) and thermodynamic modeling by prigogine-flory-patterson model," The Journal of Chemical Thermodynamics, vol. 45, no. 1, pp. 35-42, 2012 .

[138] D. Gidaspow, Y.-T. Shih, J. Bouillard, and D. Wasan, "Hydrodynamics of a lamella electrosettler," AIChE journal, vol. 35, no. 5, pp. 714-724, 1989.

[139] A. Taleb, C. Petit, and M. Pileni, "Synthesis of highly monodisperse silver nanoparticles from aot reverse micelles: a way to $2 \mathrm{~d}$ and $3 \mathrm{~d}$ self-organization," Chemistry of materials, vol. 9, no. 4, pp. 950-959, 1997.

[140] P. Alexandridis, U. Olsson, and B. Lindman, "A record nine different phases (four cubic, two hexagonal, and one lamellar lyotropic liquid crystalline and two micellar solutions) in a ternary isothermal system of an amphiphilic block copolymer and selective solvents (water and oil)," Langmuir, vol. 14, no. 10, pp. 2627-2638, 1998.

[141] C. Müller-Goymann, "Physicochemical characterization of colloidal drug delivery systems such as reverse micelles, vesicles, liquid crystals and nanoparticles for topical administration," European Journal of Pharmaceutics and Biopharmaceutics, vol. 58, no. 2, pp. 343-356, 2004. 
[142] D. Libster, P. Ben Ishai, A. Aserin, G. Shoham, and N. Garti, "From the microscopic to the mesoscopic properties of lyotropic reverse hexagonal liquid crystals," Langmuir, vol. 24, no. 5, pp. 2118-2127, 2008.

[143] P. Reymond, F. Perren, F. Lazeyras, and N. Stergiopulos, "Patient-specific mean pressure drop in the systemic arterial tree, a comparison between 1-d and 3-d models," Journal of Biomechanics, vol. 45, no. 15, pp. 2499 - 2505, 2012 .

[144] E. Maire, P. Colombo, J. Adrien, L. Babout, and L. Biasetto, "Characterization of the morphology of cellular ceramics by 3d image processing of x-ray tomography," Journal of the European Ceramic Society, vol. 27, no. 4, pp. 1973 - 1981, 2007.

[145] W. Fourie, R. Said, P. Young, and D. L. Barnes, "The simulation of pore scale fluid flow with real world geometries obtained from x-ray computed tomography," in Proceedings of the Boston COMSOL Conference, 2007.

[146] I. J. R. J. . J. A. S. . G. K. N. . et al., "Nondestructive reconstruction and analysis of sofc anodes using x-ray computed tomography at sub-50 nm resolution," JOURNAL OF THE ELECTROCHEMICAL SOCIETY, vol. 155, pp. B504-B508, 2008.

[147] Y. Sui, B. Cui, L. Martnez, R. Perez, and D. Sellmyer, "Pore structure, barrier layer topography and matrix alumina structure of porous anodic alumina film," Thin Solid Films, vol. 406, pp. 64-69, 2002.

[148] D. COMSOL, C. M. Books, C. Consultants, S. Chart, C. S. Chart, C. Multiphysics, N. Structural, and F. M. CFD, "Modeling of chloride transport in cracked concrete: a 3-d image-based microstructure simulation,"

[149] Y. Sui, B. Cui, L. Martmez, R. Perez, and D. J. Sellmyer, "Pore structure, barrier layer topography and matrix alumina structure of porous anodic alumina film," Thin Solid Films, vol. 406, no. 1, pp. 64-69, 2002.

[150] O. D. Velev and A. M. Lenhoff, "Colloidal crystals as templates for porous materials," Current Opinion in Colloid $\& 3$ Interface Science, vol. 5, pp. $56-$ 63, 2000 .

[151] I. C. for Display Metrology, Information Display Measurement Standard. Society for Information Display, 2012.

[152] J. Miseli, "24.4: Introduction to the icdm display measurement standard," SID Symposium Digest of Technical Papers, vol. 40, no. 1, pp. 332-335, 2009. 
[153] J. M. Lucas and R. B. Crosier, "Fast initial response for cusum quality-control schemes: Give your cusum a head start," Technometrics, vol. 24, no. 3, pp. 199-205, 1982.

[154] D. Montgomery, Introduction to Statistical Quality Control. Wiley Desktop Editions Series, Wiley, 2008.

[155] M. S. Srivastava and Y. Wu, "Comparison of ewma, cusum and shiryayev-roberts procedures for detecting a shift in the mean," The Annals of Statistics, vol. 21, no. 2, pp. pp. 645-670, 1993.

[156] F. Beunis, F. Strubbe, M. Karvar, O. Drobchak, T. Brans, K. Neyts, and A. R. Verschueren, "Electric charging of inverse micelles in a nonpolar liquid with surfactant," Colloids and Surfaces A: Physicochemical and Engineering Aspects, 2013. 
APPENDIX A

STEADY STATE DATA FROM CONCENTRATION EXPERIMENT 
Data A-1:

Concentration vs. luminance at $\mathbf{1 2 . 5 \%}$ ink dilution (effect of concentration on contrast response).

\begin{tabular}{|c|c|}
\hline $\begin{array}{c}\text { Concentration } \\
(\%)\end{array}$ & Luminance \\
\hline 12.5 & 0.2425 \\
\hline 12.5 & 0.2416 \\
\hline 12.5 & 0.2408 \\
\hline 12.5 & 0.2398 \\
\hline 12.5 & 0.2441 \\
\hline 12.5 & 0.2386 \\
\hline 12.5 & 0.2419 \\
\hline 12.5 & 0.2399 \\
\hline 12.5 & 0.2390 \\
\hline 12.5 & 0.2426 \\
\hline 12.5 & 0.2401 \\
\hline 12.5 & 0.2388 \\
\hline 12.5 & 0.2407 \\
\hline 12.5 & 0.2428 \\
\hline 12.5 & 0.2490 \\
\hline 12.5 & 0.2655 \\
\hline 12.5 & 0.2899 \\
\hline 12.5 & 0.3167 \\
\hline 12.5 & 0.3200 \\
\hline 12.5 & 0.3201 \\
\hline 12.5 & 0.3229 \\
\hline 12.5 & 0.3250 \\
\hline 12.5 & 0.3223 \\
\hline 12.5 & 0.3242 \\
\hline 12.5 & 0.3228 \\
\hline 12.5 & 0.3238 \\
\hline 12.5 & 0.3254 \\
\hline 12.5 & 0.3269 \\
\hline 12.5 & 0.3305 \\
\hline 12.5 & 0.3216 \\
\hline 12.5 & 0.3259 \\
\hline 12.5 & 0.3261 \\
\hline
\end{tabular}

\begin{tabular}{|c|c|}
\hline 12.5 & 0.3245 \\
\hline 12.5 & 0.3256 \\
\hline 12.5 & 0.3261 \\
\hline 12.5 & 0.3270 \\
\hline 12.5 & 0.3256 \\
\hline 12.5 & 0.3100 \\
\hline 12.5 & 0.2887 \\
\hline 12.5 & 0.2633 \\
\hline 12.5 & 0.2479 \\
\hline 12.5 & 0.2472 \\
\hline 12.5 & 0.2462 \\
\hline 12.5 & 0.2421 \\
\hline 12.5 & 0.2390 \\
\hline 12.5 & 0.2393 \\
\hline 12.5 & 0.2393 \\
\hline 12.5 & 0.2412 \\
\hline 12.5 & 0.2410 \\
\hline 12.5 & 0.2401 \\
\hline 12.5 & 0.2396 \\
\hline 12.5 & 0.2408 \\
\hline 12.5 & 0.2407 \\
\hline 12.5 & 0.2432 \\
\hline 12.5 & 0.2383 \\
\hline 12.5 & 0.2460 \\
\hline 12.5 & 0.2404 \\
\hline 12.5 & 0.2381 \\
\hline 12.5 & 0.2385 \\
\hline 12.5 & 0.2386 \\
\hline 12.5 & 0.2597 \\
\hline 12.5 & 0.2770 \\
\hline 12.5 & 0.2994 \\
\hline 12.5 & 0.3182 \\
\hline
\end{tabular}

\begin{tabular}{|c|c|}
\hline 12.5 & 0.3238 \\
\hline 12.5 & 0.3254 \\
\hline 12.5 & 0.3238 \\
\hline 12.5 & 0.3229 \\
\hline 12.5 & 0.3204 \\
\hline 12.5 & 0.3262 \\
\hline 12.5 & 0.3223 \\
\hline 12.5 & 0.3284 \\
\hline 12.5 & 0.3289 \\
\hline 12.5 & 0.3221 \\
\hline 12.5 & 0.3254 \\
\hline 12.5 & 0.3266 \\
\hline 12.5 & 0.3274 \\
\hline 12.5 & 0.3301 \\
\hline 12.5 & 0.3270 \\
\hline 12.5 & 0.3285 \\
\hline 12.5 & 0.3296 \\
\hline 12.5 & 0.3295 \\
\hline 12.5 & 0.3251 \\
\hline 12.5 & 0.3006 \\
\hline 12.5 & 0.2857 \\
\hline 12.5 & 0.2571 \\
\hline 12.5 & 0.2508 \\
\hline 12.5 & 0.2416 \\
\hline 12.5 & 0.2394 \\
\hline 12.5 & 0.2411 \\
\hline
\end{tabular}


Data A-2:

Concentration vs. luminance at $\mathbf{1 8 . 8 \%}$ ink dilution (effect of concentration on contrast response).

\begin{tabular}{|c|c|}
\hline $\begin{array}{c}\text { Concentration } \\
(\%)\end{array}$ & Luminance \\
\hline 18.8 & 0.1985 \\
\hline 18.8 & 0.1958 \\
\hline 18.8 & 0.1979 \\
\hline 18.8 & 0.1990 \\
\hline 18.8 & 0.1981 \\
\hline 18.8 & 0.1930 \\
\hline 18.8 & 0.1995 \\
\hline 18.8 & 0.1952 \\
\hline 18.8 & 0.1948 \\
\hline 18.8 & 0.1953 \\
\hline 18.8 & 0.1953 \\
\hline 18.8 & 0.1939 \\
\hline 18.8 & 0.1959 \\
\hline 18.8 & 0.1972 \\
\hline 18.8 & 0.1939 \\
\hline 18.8 & 0.2026 \\
\hline 18.8 & 0.2628 \\
\hline 18.8 & 0.2944 \\
\hline 18.8 & 0.3026 \\
\hline 18.8 & 0.3080 \\
\hline 18.8 & 0.3068 \\
\hline 18.8 & 0.3115 \\
\hline 18.8 & 0.3127 \\
\hline 18.8 & 0.3149 \\
\hline 18.8 & 0.3088 \\
\hline 18.8 & 0.3136 \\
\hline 18.8 & 0.3114 \\
\hline 18.8 & 0.3147 \\
\hline 18.8 & 0.3132 \\
\hline 18.8 & 0.3122 \\
\hline 18.8 & 0.3110 \\
\hline 18.8 & 0.3164 \\
\hline
\end{tabular}

\begin{tabular}{|c|c|}
\hline 18.8 & 0.3172 \\
\hline 18.8 & 0.3174 \\
\hline 18.8 & 0.3158 \\
\hline 18.8 & 0.3155 \\
\hline 18.8 & 0.3163 \\
\hline 18.8 & 0.3177 \\
\hline 18.8 & 0.3030 \\
\hline 18.8 & 0.2331 \\
\hline 18.8 & 0.2096 \\
\hline 18.8 & 0.2090 \\
\hline 18.8 & 0.2026 \\
\hline 18.8 & 0.1993 \\
\hline 18.8 & 0.1953 \\
\hline 18.8 & 0.1987 \\
\hline 18.8 & 0.1991 \\
\hline 18.8 & 0.1971 \\
\hline 18.8 & 0.1986 \\
\hline 18.8 & 0.1968 \\
\hline 18.8 & 0.1979 \\
\hline 18.8 & 0.1982 \\
\hline 18.8 & 0.1963 \\
\hline 18.8 & 0.1973 \\
\hline 18.8 & 0.1944 \\
\hline 18.8 & 0.1998 \\
\hline 18.8 & 0.1965 \\
\hline 18.8 & 0.1952 \\
\hline 18.8 & 0.1966 \\
\hline 18.8 & 0.1946 \\
\hline 18.8 & 0.1967 \\
\hline 18.8 & 0.2102 \\
\hline 18.8 & 0.2711 \\
\hline 18.8 & 0.2984 \\
\hline
\end{tabular}

\begin{tabular}{|ll}
\hline 18.8 & 0.3040 \\
\hline 18.8 & 0.3122 \\
\hline 18.8 & 0.3093 \\
\hline 18.8 & 0.3117 \\
\hline 18.8 & 0.3128 \\
\hline 18.8 & 0.3134 \\
\hline 18.8 & 0.3110 \\
\hline 18.8 & 0.3146 \\
\hline 18.8 & 0.3131 \\
\hline 18.8 & 0.3134 \\
\hline 18.8 & 0.3132 \\
\hline 18.8 & 0.3180 \\
\hline 18.8 & 0.3178 \\
\hline 18.8 & 0.3172 \\
\hline 18.8 & 0.3164 \\
\hline 18.8 & 0.3182 \\
\hline 18.8 & 0.3176 \\
\hline 18.8 & 0.3127 \\
\hline 18.8 & 0.3167 \\
\hline 18.8 & 0.3159 \\
\hline 18.8 & 0.2856 \\
\hline 18.8 & 0.2212 \\
\hline 18.8 & 0.2091 \\
\hline 18.8 & 0.2016 \\
\hline 18.8 & 0.1994 \\
\hline 18.8 & 0.1975 \\
\hline & \\
\hline
\end{tabular}


Data A-3:

Concentration vs. luminance at $\mathbf{2 5 \%}$ ink dilution (effect of concentration on contrast response).

\begin{tabular}{|c|c|}
\hline $\begin{array}{c}\text { Concentration } \\
(\%)\end{array}$ & Luminance \\
\hline 25.0 & 0.1666 \\
\hline 25.0 & 0.1642 \\
\hline 25.0 & 0.1662 \\
\hline 25.0 & 0.1650 \\
\hline 25.0 & 0.1626 \\
\hline 25.0 & 0.1635 \\
\hline 25.0 & 0.1633 \\
\hline 25.0 & 0.1607 \\
\hline 25.0 & 0.1672 \\
\hline 25.0 & 0.1647 \\
\hline 25.0 & 0.1629 \\
\hline 25.0 & 0.1657 \\
\hline 25.0 & 0.1654 \\
\hline 25.0 & 0.1678 \\
\hline 25.0 & 0.1675 \\
\hline 25.0 & 0.1822 \\
\hline 25.0 & 0.2563 \\
\hline 25.0 & 0.2817 \\
\hline 25.0 & 0.2903 \\
\hline 25.0 & 0.2969 \\
\hline 25.0 & 0.2945 \\
\hline 25.0 & 0.2974 \\
\hline 25.0 & 0.2967 \\
\hline 25.0 & 0.3009 \\
\hline 25.0 & 0.2978 \\
\hline 25.0 & 0.2967 \\
\hline 25.0 & 0.3008 \\
\hline 25.0 & 0.2964 \\
\hline 25.0 & 0.3009 \\
\hline 25.0 & 0.2965 \\
\hline 25.0 & 0.2982 \\
\hline 25.0 & 0.2992 \\
\hline
\end{tabular}

\begin{tabular}{|ll}
\hline $\mathbf{2 5 . 0}$ & 0.3009 \\
\hline $\mathbf{2 5 . 0}$ & 0.3000 \\
\hline $\mathbf{2 5 . 0}$ & 0.2970 \\
\hline $\mathbf{2 5 . 0}$ & 0.3026 \\
\hline $\mathbf{2 5 . 0}$ & 0.2983 \\
\hline $\mathbf{2 5 . 0}$ & 0.3022 \\
\hline $\mathbf{2 5 . 0}$ & 0.2796 \\
\hline $\mathbf{2 5 . 0}$ & 0.2015 \\
\hline $\mathbf{2 5 . 0}$ & 0.1731 \\
\hline $\mathbf{2 5 . 0}$ & 0.1694 \\
\hline $\mathbf{2 5 . 0}$ & 0.1669 \\
\hline $\mathbf{2 5 . 0}$ & 0.1664 \\
\hline $\mathbf{2 5 . 0}$ & 0.1648 \\
\hline $\mathbf{2 5 . 0}$ & 0.1652 \\
\hline $\mathbf{2 5 . 0}$ & 0.1666 \\
\hline $\mathbf{2 5 . 0}$ & 0.1655 \\
\hline $\mathbf{2 5 . 0}$ & 0.1643 \\
\hline $\mathbf{2 5 . 0}$ & 0.1693 \\
\hline $\mathbf{2 5 . 0}$ & 0.1629 \\
\hline $\mathbf{2 5 . 0}$ & 0.1643 \\
\hline $\mathbf{2 5 . 0}$ & 0.1605 \\
\hline $\mathbf{2 5 . 0}$ & 0.1657 \\
\hline $\mathbf{2 5 . 0}$ & 0.1660 \\
\hline $\mathbf{2 5 . 0}$ & 0.1662 \\
\hline $\mathbf{2 5 . 0}$ & 0.1647 \\
\hline $\mathbf{2 5 . 0}$ & 0.1631 \\
\hline $\mathbf{2 5 . 0}$ & 0.1622 \\
\hline $\mathbf{2 5 . 0}$ & 0.1628 \\
\hline $\mathbf{2 5 . 0}$ & 0.1666 \\
\hline $\mathbf{2 5 . 0}$ & 0.1857 \\
\hline $\mathbf{2 5 . 0}$ & 0.2630 \\
\hline $\mathbf{2 5 . 0}$ & 0.2872 \\
\hline & \\
\hline
\end{tabular}

\begin{tabular}{ll}
\hline $\mathbf{2 5 . 0}$ & 0.2909 \\
$\mathbf{2 5 . 0}$ & 0.2920 \\
$\mathbf{2 5 . 0}$ & 0.2911 \\
$\mathbf{2 5 . 0}$ & 0.2965 \\
$\mathbf{2 5 . 0}$ & 0.2968 \\
$\mathbf{2 5 . 0}$ & 0.2990 \\
\hline $\mathbf{2 5 . 0}$ & 0.2978 \\
\hline $\mathbf{2 5 . 0}$ & 0.2981 \\
\hline $\mathbf{2 5 . 0}$ & 0.2982 \\
$\mathbf{2 5 . 0}$ & 0.2997 \\
\hline $\mathbf{2 5 . 0}$ & 0.2972 \\
\hline $\mathbf{2 5 . 0}$ & 0.2993 \\
\hline $\mathbf{2 5 . 0}$ & 0.2973 \\
$\mathbf{2 5 . 0}$ & 0.3009 \\
\hline $\mathbf{2 5 . 0}$ & 0.3018 \\
\hline $\mathbf{2 5 . 0}$ & 0.3010 \\
\hline $\mathbf{2 5 . 0}$ & 0.2974 \\
\hline $\mathbf{2 5 . 0}$ & 0.3021 \\
\hline $\mathbf{2 5 . 0}$ & 0.3020 \\
$\mathbf{2 5 . 0}$ & 0.2994 \\
\hline $\mathbf{2 5 . 0}$ & 0.2674 \\
$\mathbf{2 5 . 0}$ & 0.1947 \\
\hline $\mathbf{2 5 . 0}$ & 0.1714 \\
$\mathbf{2 5 . 0}$ & 0.1705 \\
$\mathbf{2 5 . 0}$ & 0.1630 \\
$\mathbf{2 5 . 0}$ & 0.1630 \\
\hline & \\
\hline
\end{tabular}


Data A-4:

Concentration vs. luminance at $\mathbf{3 7 . 5 \%}$ ink dilution (effect of concentration on contrast response).

\begin{tabular}{|c|c|}
\hline $\begin{array}{c}\text { Concentration } \\
(\%)\end{array}$ & Luminance \\
\hline 37.5 & 0.1656 \\
\hline 37.5 & 0.1667 \\
\hline 37.5 & 0.1650 \\
\hline 37.5 & 0.1639 \\
\hline 37.5 & 0.1652 \\
\hline 37.5 & 0.1661 \\
\hline 37.5 & 0.1639 \\
\hline 37.5 & 0.1628 \\
\hline 37.5 & 0.1674 \\
\hline 37.5 & 0.1647 \\
\hline 37.5 & 0.1675 \\
\hline 37.5 & 0.1663 \\
\hline 37.5 & 0.1666 \\
\hline 37.5 & 0.1647 \\
\hline 37.5 & 0.1658 \\
\hline 37.5 & 0.1861 \\
\hline 37.5 & 0.2131 \\
\hline 37.5 & 0.2558 \\
\hline 37.5 & 0.2671 \\
\hline 37.5 & 0.2761 \\
\hline 37.5 & 0.2765 \\
\hline 37.5 & 0.2791 \\
\hline 37.5 & 0.2811 \\
\hline 37.5 & 0.2797 \\
\hline 37.5 & 0.2783 \\
\hline 37.5 & 0.2775 \\
\hline 37.5 & 0.2786 \\
\hline 37.5 & 0.2801 \\
\hline 37.5 & 0.2832 \\
\hline 37.5 & 0.2811 \\
\hline 37.5 & 0.2769 \\
\hline 37.5 & 0.2814 \\
\hline
\end{tabular}

\begin{tabular}{|ll}
\hline 37.5 & 0.2799 \\
\hline 37.5 & 0.2795 \\
\hline 37.5 & 0.2815 \\
\hline 37.5 & 0.2771 \\
\hline 37.5 & 0.2819 \\
\hline 37.5 & 0.2745 \\
\hline 37.5 & 0.2508 \\
\hline 37.5 & 0.2348 \\
\hline 37.5 & 0.1910 \\
\hline 37.5 & 0.1714 \\
\hline 37.5 & 0.1695 \\
\hline 37.5 & 0.1682 \\
\hline 37.5 & 0.1665 \\
\hline 37.5 & 0.1677 \\
\hline 37.5 & 0.1615 \\
\hline 37.5 & 0.1658 \\
\hline 37.5 & 0.1626 \\
\hline 37.5 & 0.1673 \\
\hline 37.5 & 0.1654 \\
\hline 37.5 & 0.1653 \\
\hline 37.5 & 0.1608 \\
\hline 37.5 & 0.1661 \\
\hline 37.5 & 0.1653 \\
\hline 37.5 & 0.1643 \\
\hline 37.5 & 0.1651 \\
\hline 37.5 & 0.1636 \\
\hline 37.5 & 0.1676 \\
\hline 37.5 & 0.1634 \\
\hline 37.5 & 0.1807 \\
\hline 37.5 & 0.1965 \\
\hline 37.5 & 0.2199 \\
\hline 37.5 & 0.2572 \\
\hline & \\
\hline
\end{tabular}

\begin{tabular}{|ll}
\hline 37.5 & 0.2707 \\
\hline 37.5 & 0.2763 \\
\hline 37.5 & 0.2720 \\
\hline 37.5 & 0.2765 \\
\hline 37.5 & 0.2812 \\
\hline 37.5 & 0.2758 \\
\hline 37.5 & 0.2793 \\
\hline 37.5 & 0.2813 \\
\hline 37.5 & 0.2827 \\
\hline 37.5 & 0.2778 \\
\hline 37.5 & 0.2803 \\
\hline 37.5 & 0.2799 \\
\hline 37.5 & 0.2797 \\
\hline 37.5 & 0.2811 \\
\hline 37.5 & 0.2741 \\
\hline 37.5 & 0.2798 \\
\hline 37.5 & 0.2786 \\
\hline 37.5 & 0.2803 \\
\hline 37.5 & 0.2803 \\
\hline 37.5 & 0.2703 \\
\hline 37.5 & 0.2503 \\
\hline 37.5 & 0.2228 \\
\hline 37.5 & 0.1879 \\
\hline 37.5 & 0.1830 \\
\hline 37.5 & 0.1696 \\
\hline 37.5 & 0.1670 \\
\hline & \\
\hline
\end{tabular}


Data A-5:

Concentration vs. luminance at $\mathbf{5 0 \%}$ ink dilution (effect of concentration on contrast response).

\begin{tabular}{|c|c|}
\hline $\begin{array}{c}\text { Concentration } \\
(\%)\end{array}$ & Luminance \\
\hline 50.0 & 0.1670 \\
\hline 50.0 & 0.1704 \\
\hline 50.0 & 0.1685 \\
\hline 50.0 & 0.1631 \\
\hline 50.0 & 0.1658 \\
\hline 50.0 & 0.1662 \\
\hline 50.0 & 0.1680 \\
\hline 50.0 & 0.1634 \\
\hline 50.0 & 0.1753 \\
\hline 50.0 & 0.1671 \\
\hline 50.0 & 0.1675 \\
\hline 50.0 & 0.1673 \\
\hline 50.0 & 0.1668 \\
\hline 50.0 & 0.1671 \\
\hline 50.0 & 0.1654 \\
\hline 50.0 & 0.1771 \\
\hline 50.0 & 0.2146 \\
\hline 50.0 & 0.2133 \\
\hline 50.0 & 0.2555 \\
\hline 50.0 & 0.2632 \\
\hline 50.0 & 0.2606 \\
\hline 50.0 & 0.2583 \\
\hline 50.0 & 0.2639 \\
\hline 50.0 & 0.2594 \\
\hline 50.0 & 0.2616 \\
\hline 50.0 & 0.2620 \\
\hline 50.0 & 0.2640 \\
\hline 50.0 & 0.2599 \\
\hline 50.0 & 0.2596 \\
\hline 50.0 & 0.2597 \\
\hline 50.0 & 0.2584 \\
\hline 50.0 & 0.2617 \\
\hline
\end{tabular}

\begin{tabular}{|ll}
\hline $\mathbf{5 0 . 0}$ & 0.2655 \\
\hline $\mathbf{5 0 . 0}$ & 0.2600 \\
\hline $\mathbf{5 0 . 0}$ & 0.2640 \\
\hline $\mathbf{5 0 . 0}$ & 0.2621 \\
\hline $\mathbf{5 0 . 0}$ & 0.2669 \\
\hline $\mathbf{5 0 . 0}$ & 0.2615 \\
\hline $\mathbf{5 0 . 0}$ & 0.2438 \\
\hline $\mathbf{5 0 . 0}$ & 0.2112 \\
\hline $\mathbf{5 0 . 0}$ & 0.2132 \\
\hline $\mathbf{5 0 . 0}$ & 0.1662 \\
\hline $\mathbf{5 0 . 0}$ & 0.1610 \\
\hline $\mathbf{5 0 . 0}$ & 0.1710 \\
\hline $\mathbf{5 0 . 0}$ & 0.1723 \\
\hline $\mathbf{5 0 . 0}$ & 0.1675 \\
\hline $\mathbf{5 0 . 0}$ & 0.1698 \\
\hline $\mathbf{5 0 . 0}$ & 0.1658 \\
\hline $\mathbf{5 0 . 0}$ & 0.1680 \\
\hline $\mathbf{5 0 . 0}$ & 0.1685 \\
\hline $\mathbf{5 0 . 0}$ & 0.1673 \\
\hline $\mathbf{5 0 . 0}$ & 0.1700 \\
\hline $\mathbf{5 0 . 0}$ & 0.1669 \\
\hline $\mathbf{5 0 . 0}$ & 0.1687 \\
\hline $\mathbf{5 0 . 0}$ & 0.1662 \\
\hline $\mathbf{5 0 . 0}$ & 0.1640 \\
\hline $\mathbf{5 0 . 0}$ & 0.1682 \\
\hline $\mathbf{5 0 . 0}$ & 0.1698 \\
\hline $\mathbf{5 0 . 0}$ & 0.1695 \\
\hline $\mathbf{5 0 . 0}$ & 0.1677 \\
\hline $\mathbf{5 0 . 0}$ & 0.1658 \\
\hline $\mathbf{5 0 . 0}$ & 0.1817 \\
\hline $\mathbf{5 0 . 0}$ & 0.2122 \\
\hline $\mathbf{5 0 . 0}$ & 0.2137 \\
\hline & \\
\hline
\end{tabular}

\begin{tabular}{ll}
\hline $\mathbf{5 0 . 0}$ & 0.2595 \\
\hline $\mathbf{5 0 . 0}$ & 0.2646 \\
$\mathbf{5 0 . 0}$ & 0.2580 \\
$\mathbf{5 0 . 0}$ & 0.2606 \\
\hline $\mathbf{5 0 . 0}$ & 0.2613 \\
\hline $\mathbf{5 0 . 0}$ & 0.2581 \\
\hline $\mathbf{5 0 . 0}$ & 0.2590 \\
\hline $\mathbf{5 0 . 0}$ & 0.2609 \\
\hline $\mathbf{5 0 . 0}$ & 0.2557 \\
\hline $\mathbf{5 0 . 0}$ & 0.2640 \\
\hline $\mathbf{5 0 . 0}$ & 0.2586 \\
\hline $\mathbf{5 0 . 0}$ & 0.2617 \\
\hline $\mathbf{5 0 . 0}$ & 0.2586 \\
\hline $\mathbf{5 0 . 0}$ & 0.2610 \\
\hline $\mathbf{5 0 . 0}$ & 0.2580 \\
\hline $\mathbf{5 0 . 0}$ & 0.2635 \\
\hline $\mathbf{5 0 . 0}$ & 0.2581 \\
\hline $\mathbf{5 0 . 0}$ & 0.2621 \\
\hline $\mathbf{5 0 . 0}$ & 0.2640 \\
\hline $\mathbf{5 0 . 0}$ & 0.2614 \\
\hline $\mathbf{5 0 . 0}$ & 0.2331 \\
\hline $\mathbf{5 0 . 0}$ & 0.2126 \\
\hline $\mathbf{5 0 . 0}$ & 0.2032 \\
$\mathbf{5 0 . 0}$ & 0.1680 \\
$\mathbf{5 0 . 0}$ & 0.1681 \\
$\mathbf{5 0 . 0}$ & 0.1703 \\
\hline & \\
\hline
\end{tabular}


Data A-6:

Concentration vs. luminance at $\mathbf{1 0 0 \%}$ ink dilution (effect of concentration on contrast response).

\begin{tabular}{|c|c|}
\hline $\begin{array}{c}\text { Concentration } \\
(\%)\end{array}$ & Luminance \\
\hline 100.0 & 0.1492 \\
\hline 100.0 & 0.1474 \\
\hline 100.0 & 0.1457 \\
\hline 100.0 & 0.1487 \\
\hline 100.0 & 0.1441 \\
\hline 100.0 & 0.1481 \\
\hline 100.0 & 0.1494 \\
\hline 100.0 & 0.1449 \\
\hline 100.0 & 0.1492 \\
\hline 100.0 & 0.1440 \\
\hline 100.0 & 0.1466 \\
\hline 100.0 & 0.1388 \\
\hline 100.0 & 0.1481 \\
\hline 100.0 & 0.1496 \\
\hline 100.0 & 0.1463 \\
\hline 100.0 & 0.1442 \\
\hline 100.0 & 0.1727 \\
\hline 100.0 & 0.1993 \\
\hline 100.0 & 0.2046 \\
\hline 100.0 & 0.2033 \\
\hline 100.0 & 0.2077 \\
\hline 100.0 & 0.2032 \\
\hline 100.0 & 0.2086 \\
\hline 100.0 & 0.2133 \\
\hline 100.0 & 0.2037 \\
\hline 100.0 & 0.2057 \\
\hline 100.0 & 0.2049 \\
\hline 100.0 & 0.2053 \\
\hline 100.0 & 0.2067 \\
\hline 100.0 & 0.2084 \\
\hline 100.0 & 0.2089 \\
\hline 100.0 & 0.2017 \\
\hline
\end{tabular}

\begin{tabular}{|c|c|}
\hline 100.0 & 0.2069 \\
\hline 100.0 & 0.2094 \\
\hline 100.0 & 0.2054 \\
\hline 100.0 & 0.2078 \\
\hline 100.0 & 0.2051 \\
\hline 100.0 & 0.2057 \\
\hline 100.0 & 0.2074 \\
\hline 100.0 & 0.1855 \\
\hline 100.0 & 0.1498 \\
\hline 100.0 & 0.1480 \\
\hline 100.0 & 0.1462 \\
\hline 100.0 & 0.1459 \\
\hline 100.0 & 0.1456 \\
\hline 100.0 & 0.1409 \\
\hline 100.0 & 0.1466 \\
\hline 100.0 & 0.1499 \\
\hline 100.0 & 0.1461 \\
\hline 100.0 & 0.1463 \\
\hline 100.0 & 0.1475 \\
\hline 100.0 & 0.1490 \\
\hline 100.0 & 0.1474 \\
\hline 100.0 & 0.1481 \\
\hline 100.0 & 0.1444 \\
\hline 100.0 & 0.1492 \\
\hline 100.0 & 0.1461 \\
\hline 100.0 & 0.1441 \\
\hline 100.0 & 0.1457 \\
\hline 100.0 & 0.1420 \\
\hline 100.0 & 0.1455 \\
\hline 100.0 & 0.1477 \\
\hline 100.0 & 0.1686 \\
\hline 100.0 & 0.2063 \\
\hline
\end{tabular}

\begin{tabular}{|ll}
\hline 100.0 & 0.2052 \\
\hline 100.0 & 0.2048 \\
\hline 100.0 & 0.2050 \\
\hline 100.0 & 0.2057 \\
\hline 100.0 & 0.2059 \\
1100.0 & 0.2050 \\
\hline 100.0 & 0.2071 \\
\hline 100.0 & 0.2051 \\
\hline 100.0 & 0.2067 \\
\hline 100.0 & 0.2071 \\
\hline 100.0 & 0.2068 \\
\hline 100.0 & 0.2091 \\
\hline 100.0 & 0.2073 \\
\hline 100.0 & 0.2112 \\
\hline 100.0 & 0.2085 \\
\hline 100.0 & 0.2111 \\
\hline 100.0 & 0.2082 \\
\hline 100.0 & 0.2045 \\
\hline 100.0 & 0.2091 \\
\hline 100.0 & 0.2103 \\
\hline 100.0 & 0.2063 \\
\hline 100.0 & 0.1861 \\
\hline 100.0 & 0.1497 \\
\hline 100.0 & 0.1444 \\
\hline 100.0 & 0.1445 \\
\hline 100.0 & 0.1482 \\
\hline & \\
\hline
\end{tabular}


Data A-7:

Resulting diagram from data A-1 to A-6

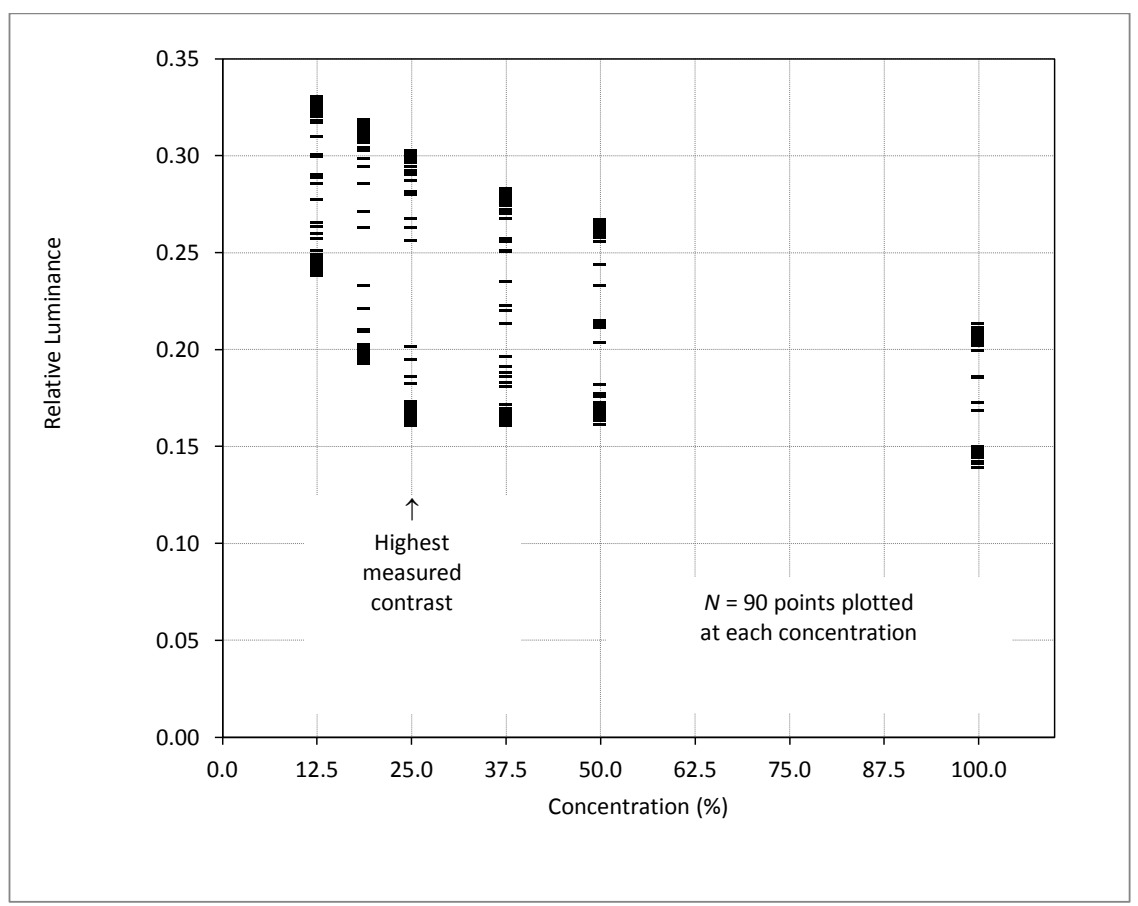




\section{APPENDIX B}

TRANSIENT DATA FROM CONCENTRATION EXPERIMENT 
Data B-1:

Data by time (effect of concentration on contrast response).

\begin{tabular}{|c|c|c|c|c|c|c|}
\hline Time (s) & $12.5 \%$ & $18.8 \%$ & $25.0 \%$ & $37.5 \%$ & $50.0 \%$ & $100 \%$ \\
\hline 0.5 & 0.2425 & 0.1985 & 0.1666 & 0.1656 & 0.1670 & 0.1492 \\
\hline 1.0 & 0.2416 & 0.1958 & 0.1642 & 0.1667 & 0.1704 & 0.1474 \\
\hline 1.5 & 0.2408 & 0.1979 & 0.1662 & 0.1650 & 0.1685 & 0.1457 \\
\hline 2.0 & 0.2398 & 0.1990 & 0.1650 & 0.1639 & 0.1631 & 0.1487 \\
\hline 2.5 & 0.2441 & 0.1981 & 0.1626 & 0.1652 & 0.1658 & 0.1441 \\
\hline 3.0 & 0.2386 & 0.1930 & 0.1635 & 0.1661 & 0.1662 & 0.1481 \\
\hline 3.5 & 0.2419 & 0.1995 & 0.1633 & 0.1639 & 0.1680 & 0.1494 \\
\hline 4.0 & 0.2399 & 0.1952 & 0.1607 & 0.1628 & 0.1634 & 0.1449 \\
\hline 4.5 & 0.2390 & 0.1948 & 0.1672 & 0.1674 & 0.1753 & 0.1492 \\
\hline 5.0 & 0.2426 & 0.1953 & 0.1647 & 0.1647 & 0.1671 & 0.1440 \\
\hline 5.5 & 0.2401 & 0.1953 & 0.1629 & 0.1675 & 0.1675 & 0.1466 \\
\hline 6.0 & 0.2388 & 0.1939 & 0.1657 & 0.1663 & 0.1673 & 0.1388 \\
\hline 6.5 & 0.2407 & 0.1959 & 0.1654 & 0.1666 & 0.1668 & 0.1481 \\
\hline 7.0 & 0.2428 & 0.1972 & 0.1678 & 0.1647 & 0.1671 & 0.1496 \\
\hline 7.5 & 0.2490 & 0.1939 & 0.1675 & 0.1658 & 0.1654 & 0.1463 \\
\hline 8.0 & 0.2655 & 0.2026 & 0.1822 & 0.1861 & 0.1771 & 0.1442 \\
\hline 8.5 & 0.2899 & 0.2628 & 0.2563 & 0.2131 & 0.2146 & 0.1727 \\
\hline 9.0 & 0.3167 & 0.2944 & 0.2817 & 0.2558 & 0.2133 & 0.1993 \\
\hline 9.5 & 0.3200 & 0.3026 & 0.2903 & 0.2671 & 0.2555 & 0.2046 \\
\hline 10.0 & 0.3201 & 0.3080 & 0.2969 & 0.2761 & 0.2632 & 0.2033 \\
\hline 10.5 & 0.3229 & 0.3068 & 0.2945 & 0.2765 & 0.2606 & 0.2077 \\
\hline 11.0 & 0.3250 & 0.3115 & 0.2974 & 0.2791 & 0.2583 & 0.2032 \\
\hline 11.5 & 0.3223 & 0.3127 & 0.2967 & 0.2811 & 0.2639 & 0.2086 \\
\hline 12.0 & 0.3242 & 0.3149 & 0.3009 & 0.2797 & 0.2594 & 0.2133 \\
\hline 12.5 & 0.3228 & 0.3088 & 0.2978 & 0.2783 & 0.2616 & 0.2037 \\
\hline 13.0 & 0.3238 & 0.3136 & 0.2967 & 0.2775 & 0.2620 & 0.2057 \\
\hline 13.5 & 0.3254 & 0.3114 & 0.3008 & 0.2786 & 0.2640 & 0.2049 \\
\hline 14.0 & 0.3269 & 0.3147 & 0.2964 & 0.2801 & 0.2599 & 0.2053 \\
\hline 14.5 & 0.3305 & 0.3132 & 0.3009 & 0.2832 & 0.2596 & 0.2067 \\
\hline 15.0 & 0.3216 & 0.3122 & 0.2965 & 0.2811 & 0.2597 & 0.2084 \\
\hline 15.5 & 0.3259 & 0.3110 & 0.2982 & 0.2769 & 0.2584 & 0.2089 \\
\hline 16.0 & 0.3261 & 0.3164 & 0.2992 & 0.2814 & 0.2617 & 0.2017 \\
\hline 16.5 & 0.3245 & 0.3172 & 0.3009 & 0.2799 & 0.2655 & 0.2069 \\
\hline 17.0 & 0.3256 & 0.3174 & 0.3000 & 0.2795 & 0.2600 & 0.2094 \\
\hline 17.5 & 0.3261 & 0.3158 & 0.2970 & 0.2815 & 0.2640 & 0.2054 \\
\hline 18.0 & 0.3270 & 0.3155 & 0.3026 & 0.2771 & 0.2621 & 0.2078 \\
\hline 18.5 & 0.3256 & 0.3163 & 0.2983 & 0.2819 & 0.2669 & 0.2051 \\
\hline 19.0 & 0.3100 & 0.3177 & 0.3022 & 0.2745 & 0.2615 & 0.2057 \\
\hline 19.5 & 0.2887 & 0.3030 & 0.2796 & 0.2508 & 0.2438 & 0.2074 \\
\hline 20.0 & 0.2633 & 0.2331 & 0.2015 & 0.2348 & 0.2112 & 0.1855 \\
\hline 20.5 & 0.2479 & 0.2096 & 0.1731 & 0.1910 & 0.2132 & 0.1498 \\
\hline 21.0 & 0.2472 & 0.2090 & 0.1694 & 0.1714 & 0.1662 & 0.1480 \\
\hline 21.5 & 0.2462 & 0.2026 & 0.1669 & 0.1695 & 0.1610 & 0.1462 \\
\hline 22.0 & 0.2421 & 0.1993 & 0.1664 & 0.1682 & 0.1710 & 0.1459 \\
\hline 22.5 & 0.2390 & 0.1953 & 0.1648 & 0.1665 & 0.1723 & 0.1456 \\
\hline 23.0 & 0.2393 & 0.1987 & 0.1652 & 0.1677 & 0.1675 & 0.1409 \\
\hline 23.5 & 0.2393 & 0.1991 & 0.1666 & 0.1615 & 0.1698 & 0.1466 \\
\hline
\end{tabular}




\begin{tabular}{|c|c|c|c|c|c|c|}
\hline 24.0 & 0.2412 & 0.1971 & 0.1655 & 0.1658 & 0.1658 & 0.1499 \\
\hline 24.5 & 0.2410 & 0.1986 & 0.1643 & 0.1626 & 0.1680 & 0.1461 \\
\hline 25.0 & 0.2401 & 0.1968 & 0.1693 & 0.1673 & 0.1685 & 0.1463 \\
\hline 25.5 & 0.2396 & 0.1979 & 0.1629 & 0.1654 & 0.1673 & 0.1475 \\
\hline 26.0 & 0.2408 & 0.1982 & 0.1643 & 0.1653 & 0.1700 & 0.1490 \\
\hline 26.5 & 0.2407 & 0.1963 & 0.1605 & 0.1608 & 0.1669 & 0.1474 \\
\hline 27.0 & 0.2432 & 0.1973 & 0.1657 & 0.1661 & 0.1687 & 0.1481 \\
\hline 27.5 & 0.2383 & 0.1944 & 0.1660 & 0.1653 & 0.1662 & 0.1444 \\
\hline 28.0 & 0.2460 & 0.1998 & 0.1662 & 0.1643 & 0.1640 & 0.1492 \\
\hline 28.5 & 0.2404 & 0.1965 & 0.1647 & 0.1651 & 0.1682 & 0.1461 \\
\hline 29.0 & 0.2381 & 0.1952 & 0.1631 & 0.1636 & 0.1698 & 0.1441 \\
\hline 29.5 & 0.2385 & 0.1966 & 0.1622 & 0.1676 & 0.1695 & 0.1457 \\
\hline 30.0 & 0.2386 & 0.1946 & 0.1628 & 0.1634 & 0.1677 & 0.1420 \\
\hline 30.5 & 0.2597 & 0.1967 & 0.1666 & 0.1807 & 0.1658 & 0.1455 \\
\hline 31.0 & 0.2770 & 0.2102 & 0.1857 & 0.1965 & 0.1817 & 0.1477 \\
\hline 31.5 & 0.2994 & 0.2711 & 0.2630 & 0.2199 & 0.2122 & 0.1686 \\
\hline 32.0 & 0.3182 & 0.2984 & 0.2872 & 0.2572 & 0.2137 & 0.2063 \\
\hline 32.5 & 0.3238 & 0.3040 & 0.2909 & 0.2707 & 0.2595 & 0.2052 \\
\hline 33.0 & 0.3254 & 0.3122 & 0.2920 & 0.2763 & 0.2646 & 0.2048 \\
\hline 33.5 & 0.3238 & 0.3093 & 0.2911 & 0.2720 & 0.2580 & 0.2050 \\
\hline 34.0 & 0.3229 & 0.3117 & 0.2965 & 0.2765 & 0.2606 & 0.2057 \\
\hline 34.5 & 0.3204 & 0.3128 & 0.2968 & 0.2812 & 0.2613 & 0.2059 \\
\hline 35.0 & 0.3262 & 0.3134 & 0.2990 & 0.2758 & 0.2581 & 0.2050 \\
\hline 35.5 & 0.3223 & 0.3110 & 0.2978 & 0.2793 & 0.2590 & 0.2071 \\
\hline 36.0 & 0.3284 & 0.3146 & 0.2981 & 0.2813 & 0.2609 & 0.2051 \\
\hline 36.5 & 0.3289 & 0.3131 & 0.2982 & 0.2827 & 0.2557 & 0.2067 \\
\hline 37.0 & 0.3221 & 0.3134 & 0.2997 & 0.2778 & 0.2640 & 0.2071 \\
\hline 37.5 & 0.3254 & 0.3132 & 0.2972 & 0.2803 & 0.2586 & 0.2068 \\
\hline 38.0 & 0.3266 & 0.3180 & 0.2993 & 0.2799 & 0.2617 & 0.2091 \\
\hline 38.5 & 0.3274 & 0.3178 & 0.2973 & 0.2797 & 0.2586 & 0.2073 \\
\hline 39.0 & 0.3301 & 0.3172 & 0.3009 & 0.2811 & 0.2610 & 0.2112 \\
\hline 39.5 & 0.3270 & 0.3164 & 0.3018 & 0.2741 & 0.2580 & 0.2085 \\
\hline 40.0 & 0.3285 & 0.3182 & 0.3010 & 0.2798 & 0.2635 & 0.2111 \\
\hline 40.5 & 0.3296 & 0.3176 & 0.2974 & 0.2786 & 0.2581 & 0.2082 \\
\hline 41.0 & 0.3295 & 0.3127 & 0.3021 & 0.2803 & 0.2621 & 0.2045 \\
\hline 41.5 & 0.3251 & 0.3167 & 0.3020 & 0.2803 & 0.2640 & 0.2091 \\
\hline 42.0 & 0.3006 & 0.3159 & 0.2994 & 0.2703 & 0.2614 & 0.2103 \\
\hline 42.5 & 0.2857 & 0.2856 & 0.2674 & 0.2503 & 0.2331 & 0.2063 \\
\hline 43.0 & 0.2571 & 0.2212 & 0.1947 & 0.2228 & 0.2126 & 0.1861 \\
\hline 43.5 & 0.2508 & 0.2091 & 0.1714 & 0.1879 & 0.2032 & 0.1497 \\
\hline 44.0 & 0.2416 & 0.2016 & 0.1705 & 0.1830 & 0.1680 & 0.1444 \\
\hline 44.5 & 0.2394 & 0.1994 & 0.1630 & 0.1696 & 0.1681 & 0.1445 \\
\hline 45.0 & 0.2411 & 0.1975 & 0.1630 & 0.1670 & 0.1703 & 0.1482 \\
\hline
\end{tabular}


Data B-2:

Data by time, result from data B-1 (effect of concentration on contrast response).

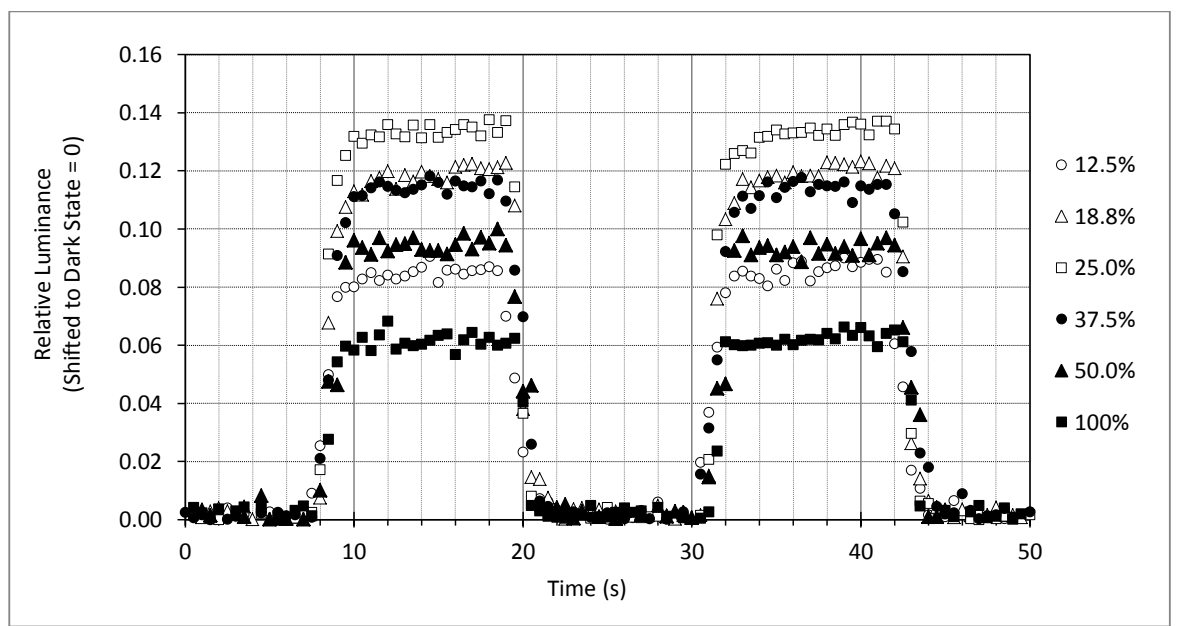


Data B-3:

Data by time, result from data B-1 (effect of concentration on contrast response) contrast.

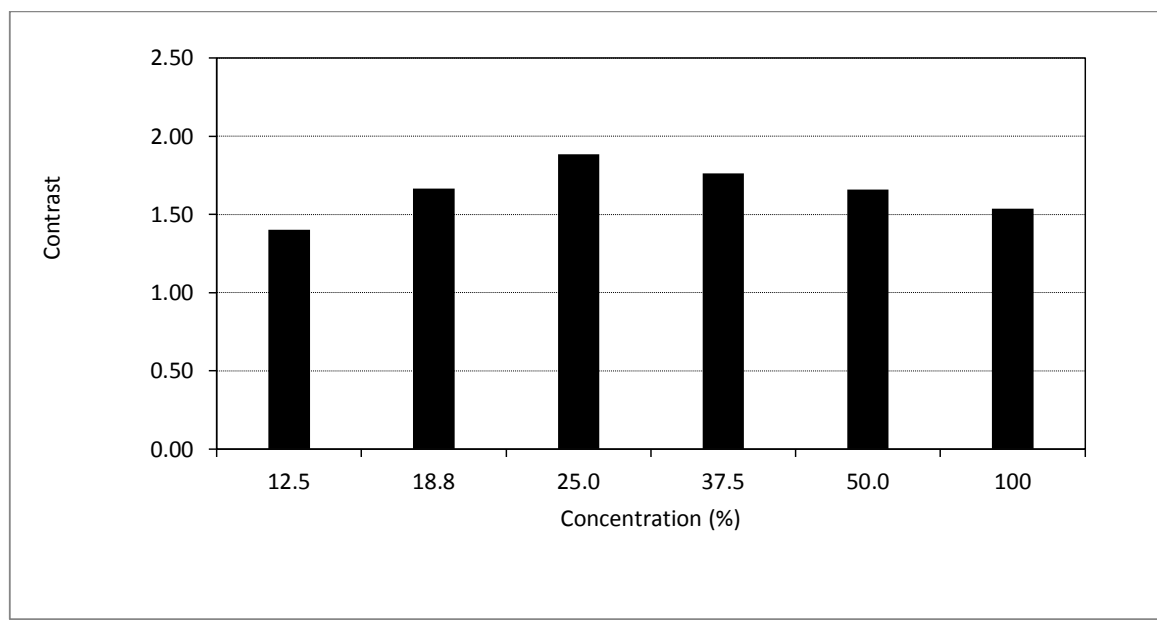




\section{APPENDIX C}

DATA FROM VOLTAGE EXPERIMENT 
Data C-1:

Data by voltage,

\begin{tabular}{|c|c|c|c|c|c|}
\hline Time (s) & & Luminance at $1 \mathrm{~V}$ & Luminance at $3 \mathrm{~V}$ & Luminance at $5 \mathrm{~V}$ & Luminance at $5 \mathrm{~V}$ \\
\hline & 0.1 & 0.2338 & 0.2081 & 0.2154 & 0.2479 \\
\hline & 0.2 & 0.2366 & 0.2106 & 0.2143 & 0.2444 \\
\hline & 0.3 & 0.2354 & 0.2115 & 0.2015 & 0.2580 \\
\hline & 0.4 & 0.2381 & 0.2121 & 0.2147 & 0.2543 \\
\hline & 0.5 & 0.2381 & 0.2091 & 0.2044 & 0.2585 \\
\hline & 0.6 & 0.2336 & 0.2147 & 0.1996 & 0.2200 \\
\hline & 0.7 & 0.2380 & 0.2316 & 0.2224 & 0.2204 \\
\hline & 0.8 & 0.2369 & 0.2362 & 0.2000 & 0.2078 \\
\hline & 0.9 & 0.2409 & 0.2395 & 0.2143 & 0.2169 \\
\hline & 1 & 0.2432 & 0.2287 & 0.2094 & 0.2063 \\
\hline & 1.1 & 0.2424 & 0.2466 & 0.2095 & 0.2068 \\
\hline & 1.2 & 0.2409 & 0.2754 & 0.2048 & 0.2049 \\
\hline & 1.3 & 0.2428 & 0.2798 & 0.2138 & 0.2032 \\
\hline & 1.4 & 0.2262 & 0.2806 & 0.2050 & 0.2135 \\
\hline & 1.5 & 0.2021 & 0.2808 & 0.2101 & 0.2005 \\
\hline & 1.6 & 0.1937 & 0.2779 & 0.2151 & 0.2415 \\
\hline & 1.7 & 0.2141 & 0.2654 & 0.2040 & 0.2565 \\
\hline & 1.8 & 0.1992 & 0.2576 & 0.1938 & 0.2475 \\
\hline & 1.9 & 0.2062 & 0.2541 & 0.2012 & 0.2396 \\
\hline & 2 & 0.2413 & 0.2507 & 0.2223 & 0.2368 \\
\hline & 2.1 & 0.2570 & 0.2520 & 0.2662 & 0.2522 \\
\hline & 2.2 & 0.2449 & 0.2465 & 0.2835 & 0.2542 \\
\hline & 2.3 & 0.2519 & 0.2229 & 0.2805 & 0.2400 \\
\hline & 2.4 & 0.2424 & 0.2144 & 0.2829 & 0.2402 \\
\hline & 2.5 & 0.2464 & 0.2134 & 0.2909 & 0.2782 \\
\hline & 2.6 & 0.2448 & 0.2071 & 0.2858 & 0.2764 \\
\hline & 2.7 & 0.2470 & 0.2013 & 0.2825 & 0.2922 \\
\hline & 2.8 & 0.2532 & 0.2087 & 0.2660 & 0.2893 \\
\hline & 2.9 & 0.2458 & 0.2084 & 0.2819 & 0.2805 \\
\hline & 3 & 0.2451 & 0.2294 & 0.2853 & 0.2873 \\
\hline & 3.1 & 0.2439 & 0.2286 & 0.2839 & 0.2905 \\
\hline & 3.2 & 0.2347 & 0.2260 & 0.2912 & 0.2927 \\
\hline & 3.3 & 0.2437 & 0.2273 & 0.2858 & 0.2910 \\
\hline & 3.4 & 0.2544 & 0.2403 & 0.2875 & 0.2776 \\
\hline & 3.5 & 0.2450 & 0.2738 & 0.2836 & 0.2592 \\
\hline & 3.6 & 0.2373 & 0.2745 & 0.2846 & 0.2456 \\
\hline & 3.7 & 0.2689 & 0.2768 & 0.2843 & 0.2488 \\
\hline & 3.8 & 0.2665 & 0.2624 & 0.2864 & 0.2540 \\
\hline & 3.9 & 0.2759 & 0.2808 & 0.2728 & 0.2661 \\
\hline & 4 & 0.2905 & 0.2729 & 0.2148 & 0.2476 \\
\hline & 4.1 & 0.2846 & 0.2595 & 0.1933 & 0.2552 \\
\hline & 4.2 & 0.2874 & 0.2457 & 0.2055 & 0.2608 \\
\hline & 4.3 & 0.2557 & 0.2552 & 0.1982 & 0.2579 \\
\hline & 4.4 & 0.2442 & 0.2485 & 0.1956 & 0.2238 \\
\hline & 4.5 & 0.2518 & 0.2530 & 0.2115 & 0.2090 \\
\hline
\end{tabular}




\begin{tabular}{rllll}
\hline $\mathbf{4 . 6}$ & 0.2391 & 0.2172 & 0.2049 & 0.2127 \\
$\mathbf{4 . 7}$ & 0.2414 & 0.2233 & 0.2078 & 0.2120 \\
$\mathbf{4 . 8}$ & 0.2362 & 0.2049 & 0.1990 & 0.2051 \\
$\mathbf{4 . 9}$ & 0.2460 & 0.2112 & 0.1986 & 0.2146 \\
$\mathbf{5}$ & 0.2510 & 0.2071 & 0.2143 & 0.2108 \\
$\mathbf{5 . 1}$ & 0.2467 & 0.2041 & 0.2062 & 0.2178 \\
$\mathbf{5 . 2}$ & 0.2493 & 0.2095 & 0.2050 & 0.2112 \\
$\mathbf{5 . 3}$ & 0.2412 & 0.2353 & 0.2030 & 0.2181 \\
$\mathbf{5 . 4}$ & 0.2466 & 0.2301 & 0.2051 & 0.2428 \\
$\mathbf{5 . 5}$ & 0.2393 & 0.2306 & 0.2070 & 0.2506 \\
$\mathbf{5 . 6}$ & 0.2435 & 0.2357 & 0.2052 & 0.2460 \\
$\mathbf{5 . 7}$ & 0.2414 & 0.2331 & 0.1878 & 0.2518 \\
$\mathbf{5 . 8}$ & 0.2450 & 0.2554 & 0.2278 & 0.2524 \\
$\mathbf{5 . 9}$ & 0.2355 & 0.2740 & 0.2835 & 0.2551 \\
$\mathbf{6}$ & 0.2197 & 0.2773 & 0.2696 & 0.2502 \\
\hline
\end{tabular}


Data C-2:

Data by voltage, contrast response, and resulting plot showing devices switched at $1 \mathrm{~V}, 3 \mathrm{~V}, 5 \mathrm{~V}, 8 \mathrm{~V}$.

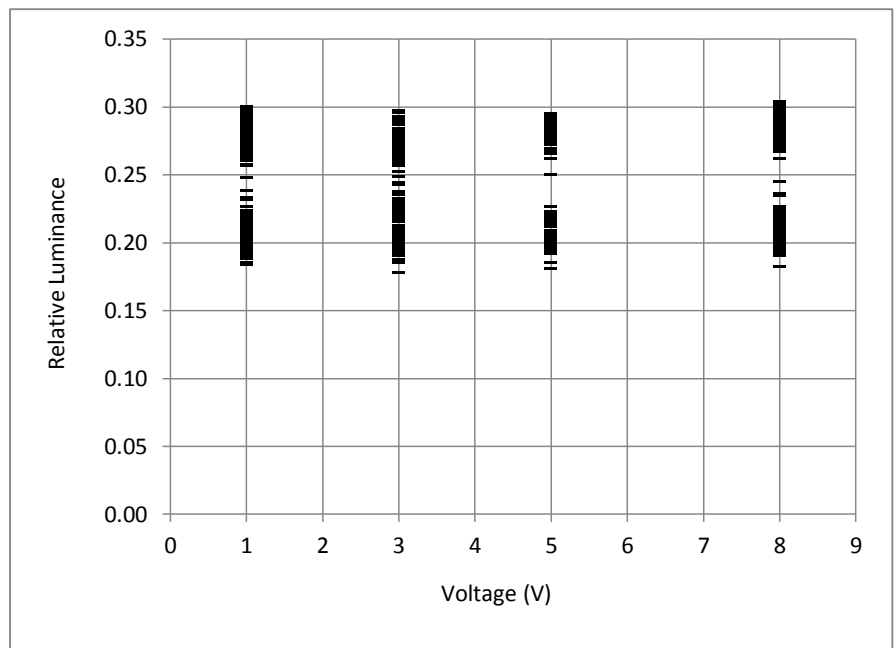


Data C-3:

C-1 data Imported with MATLAB.
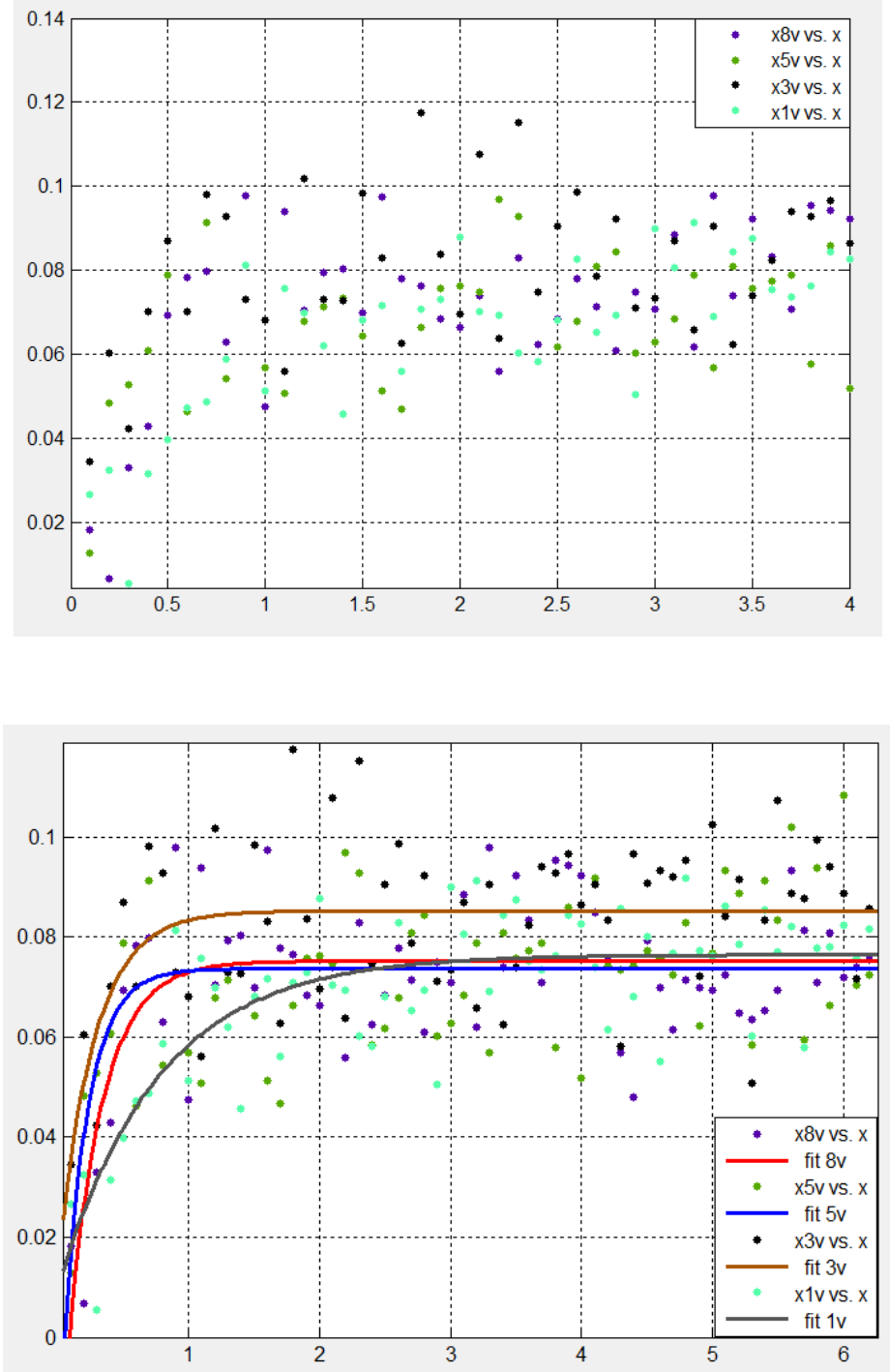
Data C-4-1:

1 V data Imported with MATLAB.

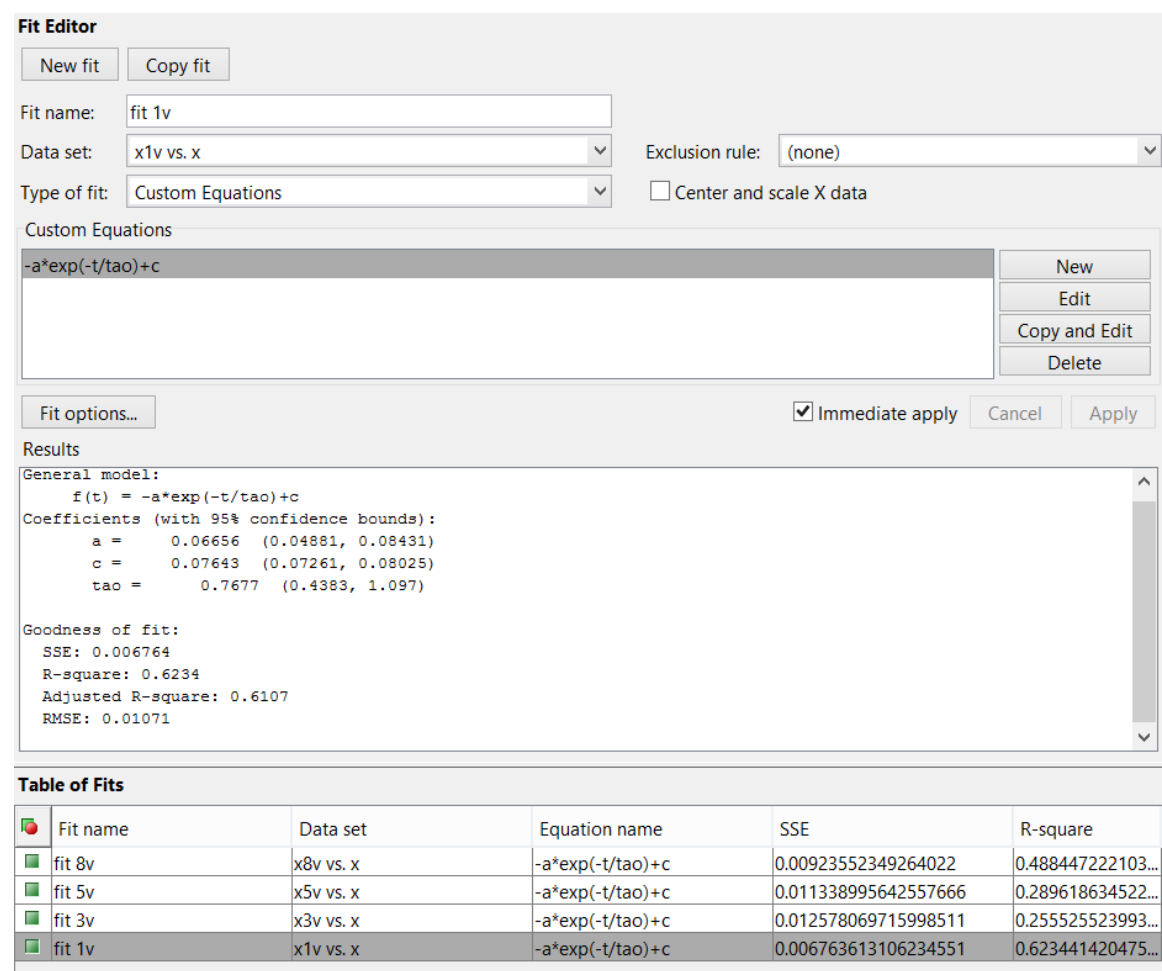


Data C-4-2:

3 V data Imported with MATLAB.

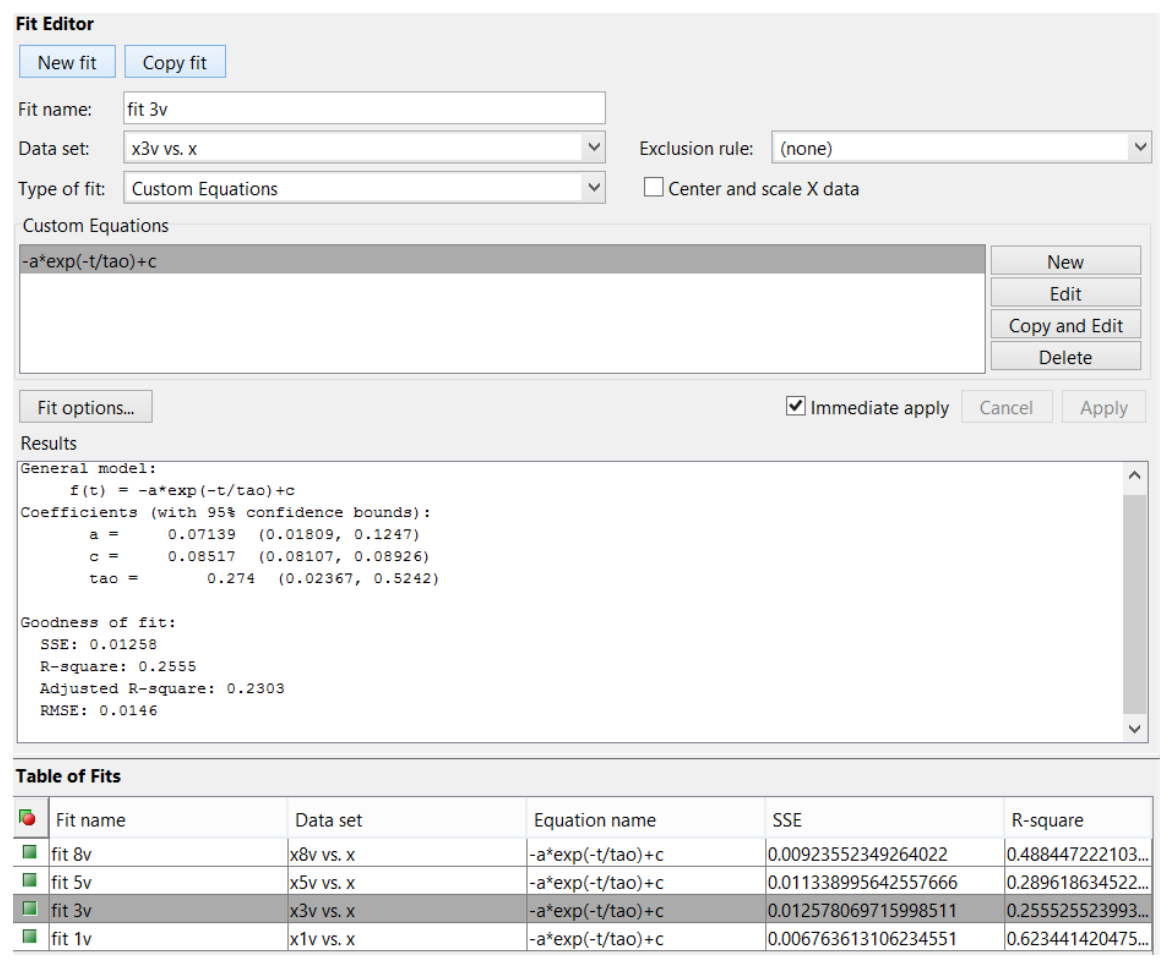


Data C-4-3:

5 V data Imported with MATLAB.

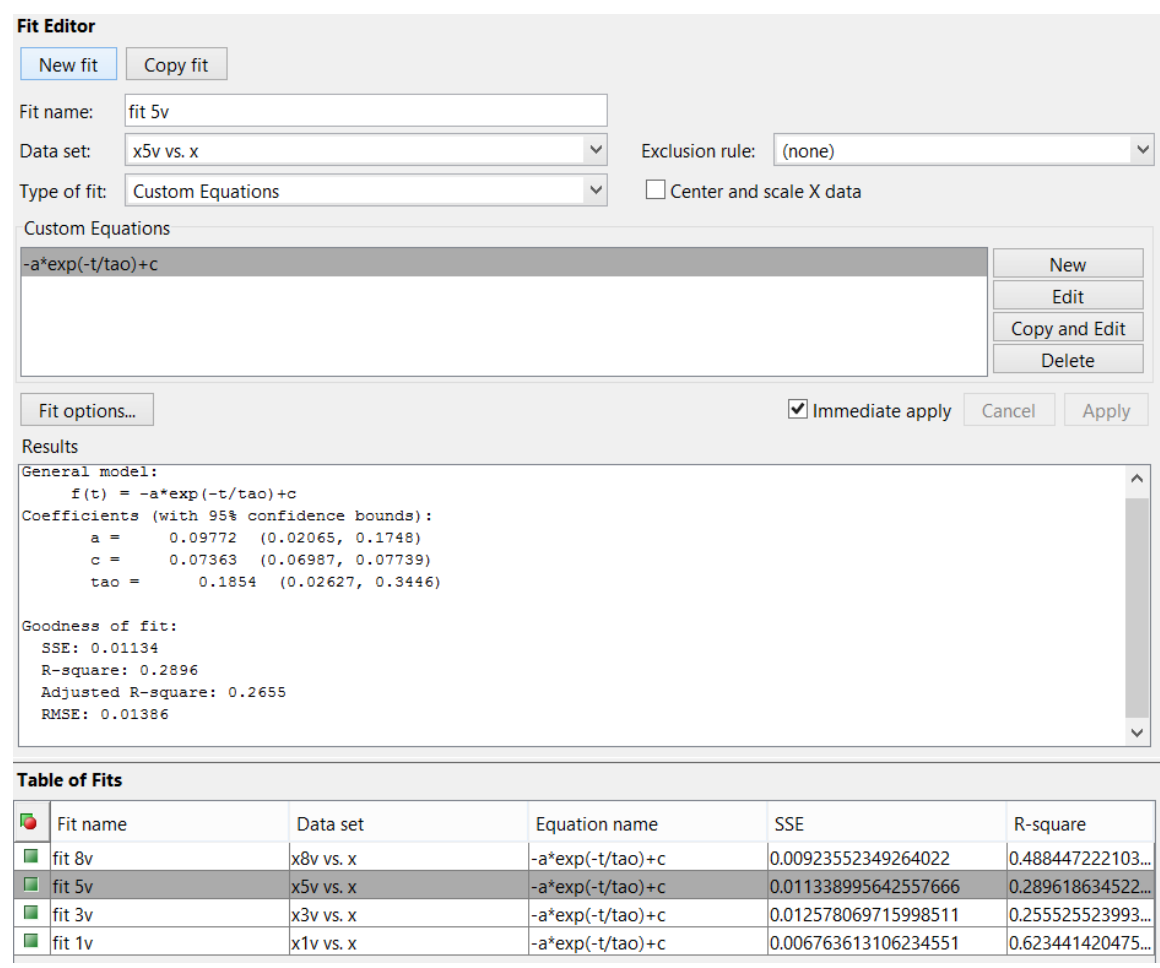


Data C-4-3:

8 V data Imported with MATLAB.

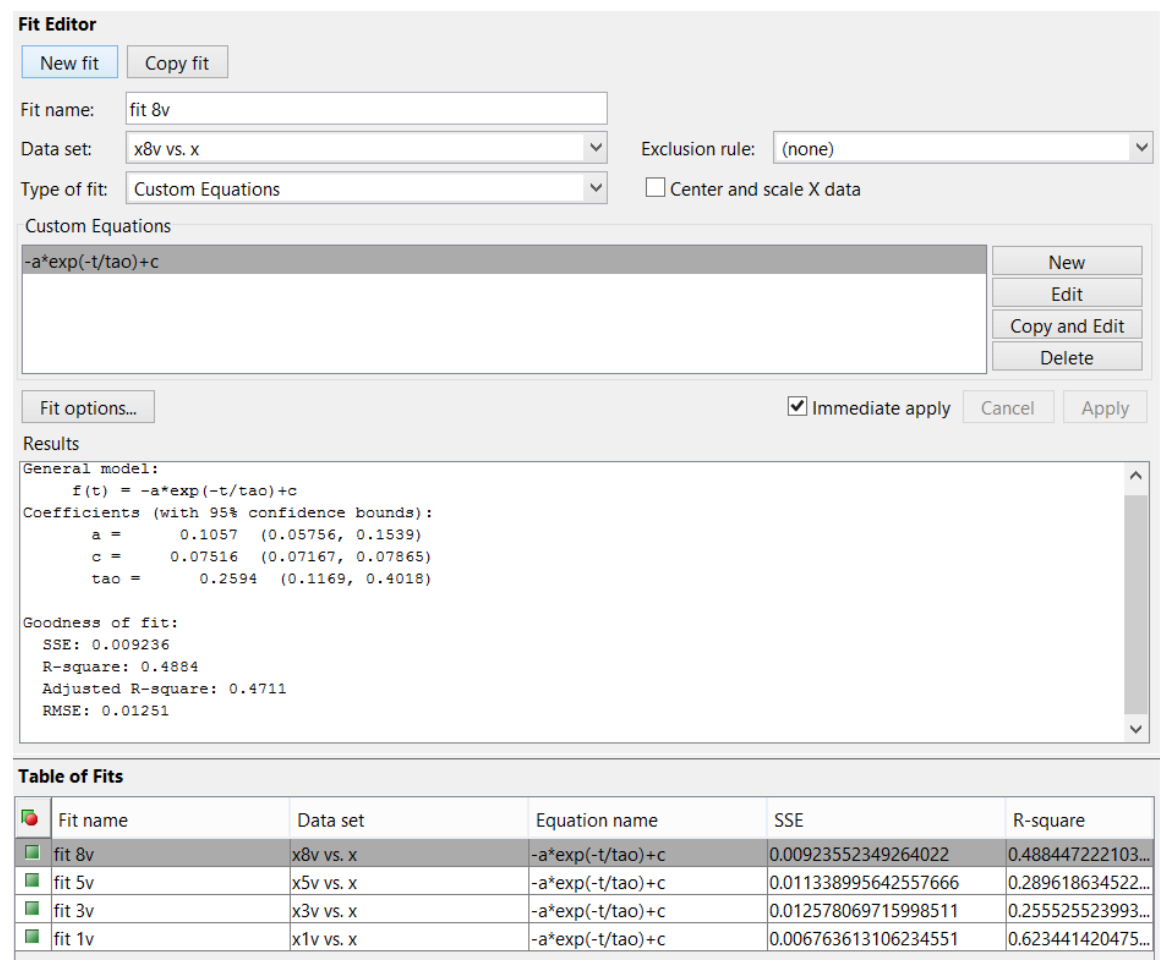




\section{APPENDIX D}

\section{DATA ANALYSIS OF THE TRANSIENT VOLTAGE EXPERIMENT}

Detecting the voltage change is the first step in finding the time constant which is the time for the luminance to rise to a percent of the saturated value. A consistent, organized, and logical method had to be used throughout the data analysis. A statistical cumulative sum control chart is used to detect reverse voltage that drives the nanodroplet away from the viewing plane. Cumulative sum (Cusum) control charts are helpful in determining small shifts within the data [153]. Cusum directly incorporates all the information by plotting the cumulative deviations from the target value. Another advantage of using the Cusum chart is the effectiveness it has with data that contains low sample size. Cusum is often used in chemical and process industries where rational subgroups are one [154]. Statistical analysis research done by Srivatava and Wu have also shown that Cusum is more efficient in detecting change in the drift than using exponentially weighted moving average (EWMA) or the Shiryayev-Roberts method [155].

For convenience, data from a step section of the square wave is cropped. This section starts from one second after the luminance has reached saturation in the state (passing the dark-to-light transition), and ends at one second before it

switches back to the dark state (which is similar to figure 37 ). Variability in relative luminance at three distinct locations per device, and for each concentration level is generated at each voltage level. The cumulative sum control chart is formed by plotting the quantity 


$$
C_{i}=\sum_{j=1}^{i}\left(\bar{x}_{j}-\mu_{0}\right)
$$

Where $n$ is the number of samples in the data, $\bar{x}_{j}$ is the average of the $j$ th sample, and $\mu_{0}$ is the targeting value determined by averaging the first segment of the step, two seconds before the transition zone takes place. One of the most fundamental Cusum is the tabular Cusum. Tabular Cusum works by accumulating positive and negative shifts into one statistic $C^{+}$and $C^{-}$respectively. The equations of $C^{+}$, and $C^{-}$are as follows.

$$
C_{i}^{+}=\max \left[0, x_{i}-\left(\mu_{0}+K\right)+C_{i-1}^{+}\right]
$$

$$
C_{i}^{-}=\max \left[0,\left(\mu_{0}-K\right)-x_{i}+C_{i-1}^{-}\right]
$$

The starting values are $C_{i}^{+}=C_{i}^{-}=0$, and $K$ is usually called the reference value (or the allowance, or slack value), [154]. $\mathrm{K}$ is chosen about halfway between the target $\mu_{0}$ and the out-of-control value of the mean, $\mu_{1}$ which needs to be detected quickly. Thus, if the shift is expressed in standard deviation units as

$$
\mu_{1}=\mu_{0}+\delta \sigma\left(\text { or } \delta=\frac{\left|\mu_{1}+\mu_{0}\right|}{\delta}\right)
$$

, then $K$ is one-half the magnitude of the shift or

$$
K=\frac{\delta}{2} \sigma=\frac{\left|\mu_{1}-\mu_{0}\right|}{2}
$$

For example, if the process outcome exceeds the reference value $K, C_{i}^{+}$will accumulate. The same thing would happen if the process outcome were to go under the reference value, $C_{i}^{-}$. Both quantities reset to zero on becoming negative. If 
either $C_{i}^{+}$or $C_{i}^{-}$exceed the decision interval $\mathrm{H}$, the process is considered out-of-control. The reasonable decision interval is 5 times the process standard deviation $\delta$. The MATLAB curve fit tool is used to find the time constant at each voltage recorded. The generalized function is as follows

$$
L=c-a * e x p^{-\frac{t}{\tau}}
$$

Equation 14

The curve fitting result showed that the device powered with $1 \mathrm{~V}$ had a longer time constant than $3 \mathrm{~V}$ and $5 \mathrm{~V}$. This means the response time is longer at lower voltage. However, device powered with $8 \mathrm{~V}$, did not show a significantly faster response than when powered by $5 \mathrm{~V}$. Research findings have shown reverse micelles surfactant becoming unstable when a higher voltage is applied [115, 156].

Researchers explained this phenomenon as the nanodroplets have the tendency to dissociate at higher voltage. Nanodroplet dissociation will lead to overall lower mobility within a device [56]. Lowering of the overall mobility at higher voltage is present in this experiment. However, further analyses need to be conducted to fully explain the physics behind the phenomenon for the ink nanodroplets. 
Data C-5:

\section{MATLAB algorisms}

function createFit $(x, x 8 v, x 5 v, x 3 v, x 1 v)$

CREATEFIT Create plot of data sets and fits.

CREATEFIT ( $\mathrm{X}, \mathrm{X} 8 \mathrm{~V}, \mathrm{X} 5 \mathrm{~V}, \mathrm{X} 3 \mathrm{~V}, \mathrm{X} 1 \mathrm{~V})$

Creates a plot, similar to the plot in the main Curve Fitting Tool, using the data that you provide as input. You can

use this function with the same data you used with CFTOOI

or with different data. You may want to edit the function to

customize the code and this help message.

Number of data sets: 4

Number of fits: 4

Data from data set "x8v vs. x":

$\mathrm{X}=\mathrm{x}:$

$\mathrm{Y}=\mathrm{x} 8 \mathrm{v}$ :

Unweighted

Data from data set "x5v vs. $x "$ :

$X=x:$

$\mathrm{Y}=\mathrm{x} 5 \mathrm{v}$ :

Unweighted

Data from data set "x3v vs. x":

$\mathrm{X}=\mathrm{x}$ :

$\mathrm{Y}=\mathrm{x} 3 \mathrm{v}:$

Unweighted

․ Data from data set "x1v vs. $x "$ :

$\mathrm{X}=\mathrm{x}$ :

$\mathrm{Y}=\mathrm{x} 1 \mathrm{v}$ :

Unweighted

\% Auto-generated by MATLAB on 09-May-2013 09:10:24

: set up figure to receive data sets and fits

$\mathrm{f}=\mathrm{clf}$;

figure (f_);

set(f_,'Units','Pixels','Position', [848 284824 589]);

을 Line handles and text for the legend.

legh $=[]$;

legt ${ }^{-}=\{\} ;$

o Limits of the $x$-axis.

$\mathrm{x} \lim =[\operatorname{Inf}-\operatorname{Inf}] ;$

을 for the plot.

ax_ $=$ axes;

set (ax,' 'Units', 'normalized', 'OuterPosition', [0 $\left.\begin{array}{llll}0 & 0 & 1 & 1\end{array}\right]$ );

set (ax,'Box', 'on');

grid (ax_,' 'on');

axes (ax_);

hold on;

\% --- Plot data that was originally in data set "x8v vs. $\mathrm{x}$ "

$\mathrm{x}=\mathrm{x}(:) ;$

$\mathrm{x} 8 \mathrm{v}=\mathrm{x} 8 \mathrm{v}(:)$

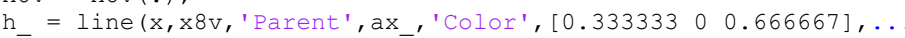

'Linestyle', 'none', 'LineWidth', 1, ...

'Marker', '.', 'MarkerSize', 12); 


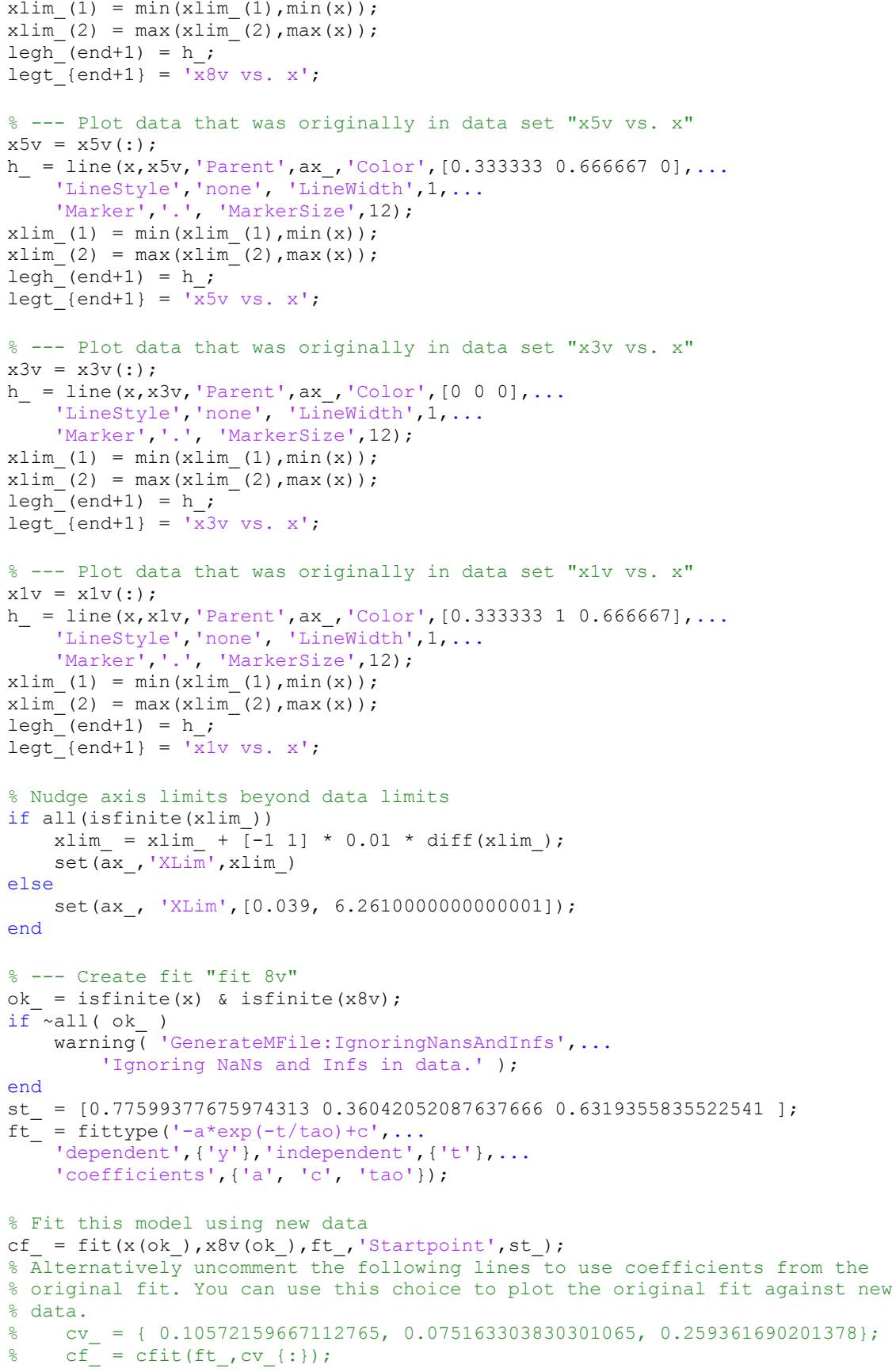




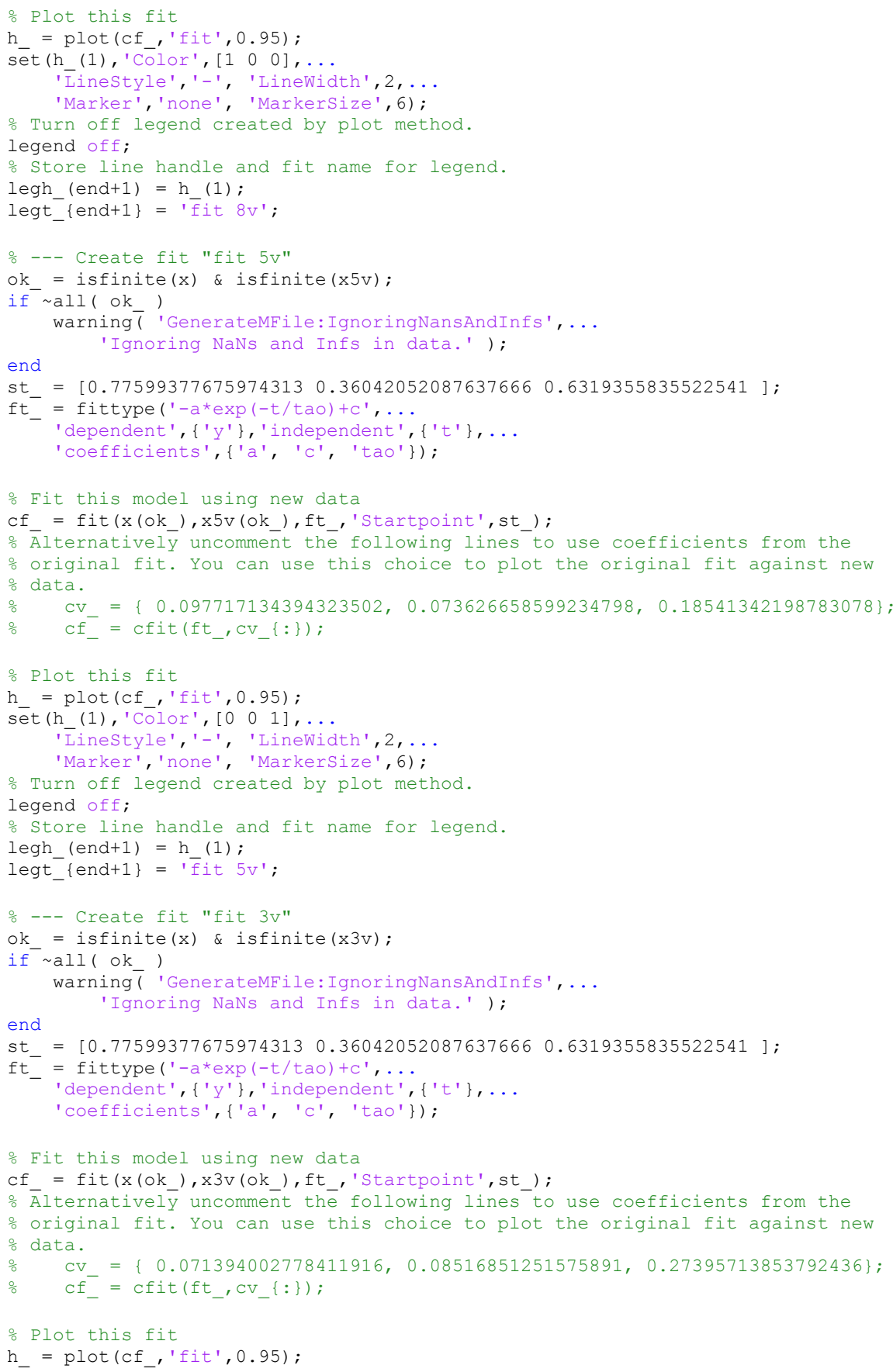




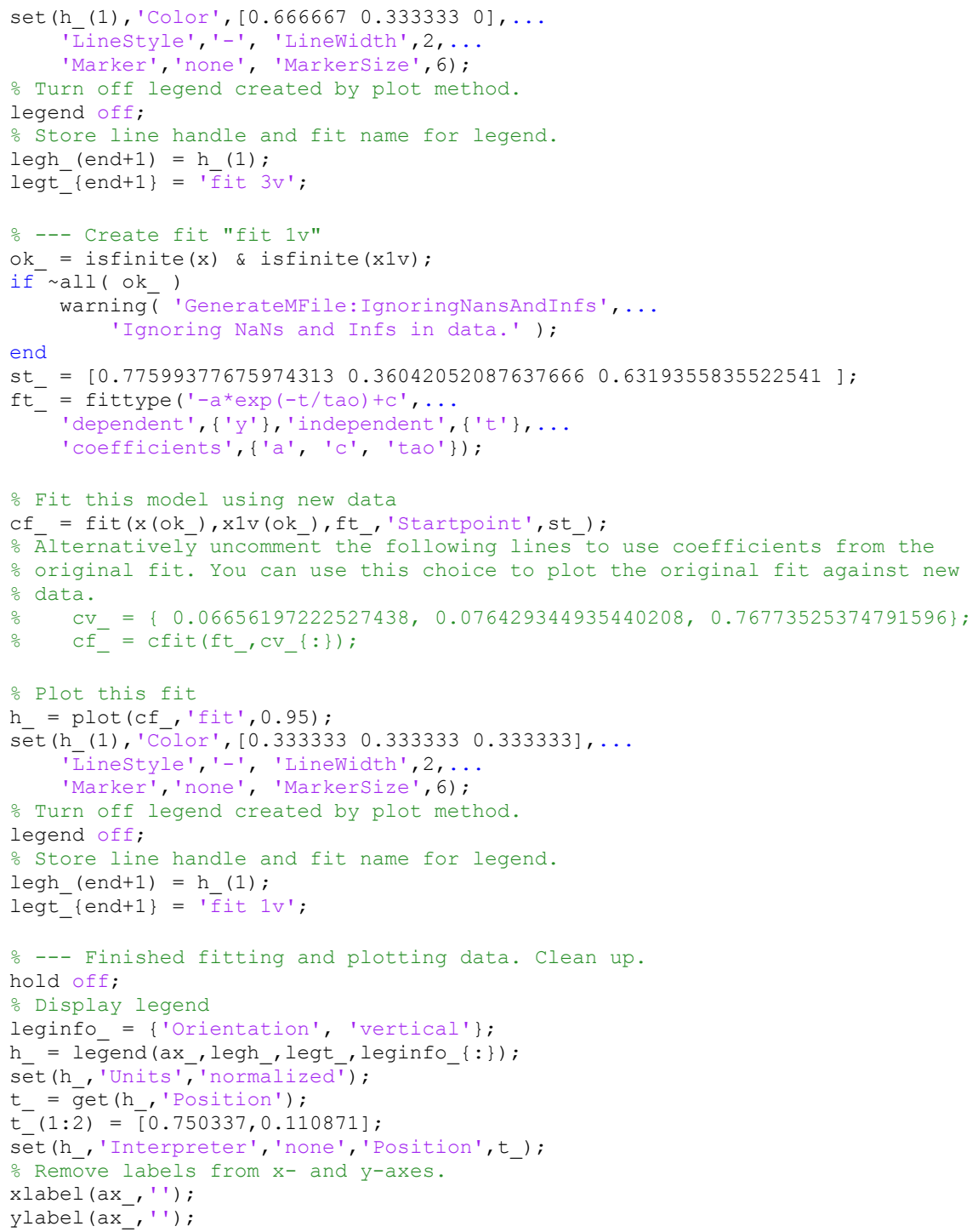

